\title{
O Teorema de Aizenman-Barsky-Fernández e a Unicidade da Temperatura Crítica
}

\author{
por \\ Jamer Insupe Roldan Gonzales \\ Orientador: Leandro Martins Cioletti
}

Brasília

2014 



\section{Jamer Insupe Roldan Gonzales}

\section{O Teorema de \\ Aizenman-Barsky-Fernández e a Unicidade da Temperatura Crítica}

Dissertação apresentada ao Programa de Pós-Graduação em Matemática da Universidade de Brasília como requisito parcial à obtenção do Título de Mestre em Matemática.

Orientador: Leandro Martins Cioletti 


Roldan, Jamer
O Teorema de Aizenman-Barsky-Fernández
e a Unicidade da Temperatura Crítica
92 páginas
Dissertação (Mestrado em Matemática).
Universidade de Brasília. Programa de Pós-
Graduação em Matemática.
1. Modelo de Ising
2. Mecânica Estatística
I. Universidade de Brasília. Departamento de
Matemática.

\section{Comissão Julgadora:}

Catia Regina Gonçalves

UnB

Rodrigo Bissacot Proença USP

Leandro Martins Cioletti

UnB 


\section{Agradecimentos}

A Deus por dar-me a vida que tenho, por fazer que hoje esteja escrevendo isto, por mostrar-me sempre uma solução aos problemas, quando há, e por dar-me sabedoria para entender que as derrotas não são mais que sinais que permitem ver que há outras formas de fazer o que se quer.

A meu orientador Dr. Leandro Martins Cioletti por sua grande paciência e compreensão para comigo, por sua amabilidade no seu trato, por seu apoio tanto intelectual como moral e pela confiança que ele atribuiu a mim; e agradeço também aqui a sua bondosa esposa Kelvia Guedes por o gesto nobre para comigo. Agradeço profundamente a professora Dra. Liliane Almeida Maia pelo seu grande apoio e confiança quando mais precisei no Brasil.

A minha família, a meus pais Mercedes e Javier, a eles devo tudo o que sou hoje. Agradeço a Aurora(Nena), Merja, Nieggy, Teylor, Juki, Ignacio por todo o apoio, carinho e força que me dão para seguir adiante. Agradeço a Efren, Elva, Wendy, Marcia, Ambrosia, Tito por sua generosidade para comigo.

A todos meus amigos da pós-graduação, especialmente a: Roberto Vila Gabriel pelo seu apoio moral, sua paciência para comigo e pela sua ajuda com as imagens do trabalho. A Jose Paiva, a Elaine, Leonardo, Christe, Valter, Lumena pelas suas sugestões, pela ajuda com a tradução, principalmente pelo entusiasmo e confiança que me proporcionam. Agradeço também a meus amigos Javier, Jaime, Roy, Jose(Charahua), Juan Carlos(Juanca), Julian, Karina, Karol pelo ânimo, e pelos bons momentos que me fizeram passar embora a distância. 


\section{Resumo}

Os principais resultados desta dissertação são baseados no artigo de M. Aizenman, D. J. Barsky, e R. Fernández intitulado: The Phase Transition in a Geral Class of Ising-Type Models is Sharp. O objetivo central é mostrar a prova de dois teoremas: O primeiro (na ordem do artigo) mostra que, numa classe geral de modelos de spins (tipo Ising estão incluídos), a temperatura crítica para susceptibilidade e para a magnetização coincidem. O segundo teorema, que implica o primeiro, estabelece importantes desigualdades diferenciais nessa classe geral de modelos, que em no casso do modelo de spins de Ising é dada por

$$
M \leq \beta h \chi+M^{3}+\beta M^{2} \frac{\partial M}{\partial \beta} .
$$

Também mostramos a prova da desigualdade de Simon-Lieb baseada no artigo de B. Simon intitulado: Correlation inequalities and the decay of correlations in ferromagnets.

Palavras-chave: Medidas de Gibbs, Modelo de Ising, Desigualdades de Correlação, Unicidade da Temperatura crítica, Correntes Aleatórias. 


\begin{abstract}
The main results of this master's thesis are based on the M. Aizenman, D. J. Barsky, and R. Fernández's paper named: The Phase Transition in a Geral Class of Ising-Type Models is Sharp. The aim is to present the proof of two theorems: the first one states that in a general class of spins models (Ising type are included), the critical temperature for susceptibility and magnetization are the same. The second theorem, which implies the first one, is about certain differential inequalities for that general class of models, which in the Ising model reads

$$
M \leq \beta h \chi+M^{3}+\beta M^{2} \frac{\partial M}{\partial \beta} .
$$

we also show the proof of the Simon-lieb inequality based on the paper by B. Simon whose title is: Correlation inequalities and the decay of correlations in ferromagnets.
\end{abstract}

Keywords: Gibbs measures, Ising Model, Correlation Inequalities, Uniqueness of the critical temperature, Random Currents. 


\section{Introdução}

No final do século XIX se deu boa parte parte do desenvolvimento do que se sabe sobre a magnetização. Na década de 20 se iniciou o estudo dos materiais ferromagnéticos, que são materiais cujos momentos magnéticos de seus elétrons ficam ordenados magneticamente, quando são submetidos a um campo magnético externo, e permanecem magnetizados por um certo tempo, mesmo após a remoção da ação do campo externo. Materiais que têm essas propriedades são, por exemplo, o Ferro e o Níquel. Vale ressaltar que a Magnetita é o material mais abundante com tal propriedade.

Para explicar os fenômenos magnéticos desses materiais foram criados certos modelos que usavam grafos para representar a estrutura cristalina dos materiais. Em tais modelos a cada vértice é associada uma variável aleatória representado o momento magnético de um átomo, que normalmente é chamada de spin.

Entre esses modelos temos o Modelo de Ising, criado em 1920 por Whilem Lenz e usado na tese de seu aluno de Doutorado Ernst Ising em 1925 [22]. Esse é o modelo mais simples capaz de modelar as propriedades magnéticas desses materiais. Com esse modelo podemos estudar propriedades macroscópicas de um sistema de partículas através de medidas de probabilidade que são definidas a partir das interações entre as partículas do sistema [21].

Um dos fenômenos mais importantes que tais modelos explicam é o fenômeno de transição de fase. No modelo de Ising a transição de fase se manifesta por uma quebra de uma simetria natural que o modelo deveria apresentar. O parâmetro de ordem capaz de identificar essa quebra de simetria é a magnetização espontânea [3], dada por

$$
M_{+}(\beta) \equiv \lim _{h \rightarrow 0^{+}}\left\langle\sigma_{0}\right\rangle_{\beta, h}
$$

Neste trabalho vamos abordar o problema de definir medidas de Gibbs a volume infinito e a transição de fase usando o formalismo desenvolvido por Dobrushin-Lanford-Ruelle (DLR). Vamos ver também uma prova para a Desigualdade de Simon-Lieb baseada no artigo Correlation Inequalities and the Decay of Correlations in Ferromagnets de Barry Simon, publicado em 1980. O trabalho de Simon representa um grande avanço na caracterização do ponto crítico, ponto onde ocorre a transição de fase do modelo de Ising ferromagnético, e foi motivado por uma tentativa de Simon de 
entender precisamente os resultados anunciados por Dobrushin no verão de 1979 [20].

Em Sharpness of the Phase Transition in Percolation Models de Michael Aizenman e David Barsky em 1987 [2], provam a igualdade de dois pontos críticos do modelo de percolação de elos independentes $\left(p_{t}\right.$ e $\left.p_{h}\right)$. Esses pontos críticos são respectivamente os pontos críticos para: a probabilidade de percolação e do valor esperado do tamanho da componente conexa da origem. Nesse trabalho é provada a igualdade desses pontos críticos para todo modelo de percolação de elos independentes invariante por translações na rede hipercúbica $d$-dimensional $(d \geq 1)$. Esses dois conceitos de ponto crítico em percolação são relacionados aos regimes de alta $(p \rightarrow 1)$ e baixa densidades $(p \rightarrow 0)$. Temos um parâmetro de or$\operatorname{dem} M(\beta, h)$, cuja versão reduzida é $M(p)=\operatorname{Prob}(|C(0)|<\infty)$, e também temos que $\chi(p)=\langle|C(0)|\rangle$. O regime de alta densidade é caracterizado por $p_{t}=\sup \{p \in[0,1]: \chi(p)<\infty\}$, enquanto o regime de baixa densidade é determinado por $p_{h}=\inf \{p \in[0,1]: M(p)>0\}$. Os principais resultados de Aizenman e Barsky são obtidos através do estudo de duas Desigualdades Diferenciais Parciais (DDP) não lineares que são:

$$
\text { (1) } M \leq h \frac{\partial M}{\partial h}+M^{2}+\beta M \frac{\partial M}{\partial \beta},
$$

$\mathrm{e}$

$$
\text { (2) } \frac{\partial M}{\partial \beta} \leq|J| M \frac{\partial M}{\partial h} .
$$

Nesta dissertação detalhamos os resultados do artigo The Phase Transition in a General Class of Ising-Type Models is Sharp [3], onde usaremos algumas ideias do artigo anterior sobre percolação. Algumas desigualdades análogas às mostradas acima são obtidas para modelos de Ising e outros sistemas ferromagnéticos na classe de Grifiths-Simon.

Vamos apresentar a dedução das desigualdades diferenciais parciais e o resultado de Aizenman-Barsky-Fernández na rede hipercúbica $d$-dimensional, $\mathbb{Z}^{d}$. O Hamiltoniano é dado por

$$
H(\sigma)=-\frac{1}{2} \sum_{x, y} J(x-y) \sigma_{x} \sigma_{y}-h \sum_{x} \sigma_{x},
$$

onde $J_{z} \geq 0$. A escolha da rede sendo $\mathbb{Z}^{d}$ é feita por questão de simplicidade pela riqueza de simetrias e também por sua importância em aplicações físicas. Como de praxe vamos assumir a hipótese de estabilidade 
das interações que no nosso caso se reduz a seguinte condição sobre as constantes de acoplamento $J(x-y)$,

$$
|J|=\sum_{x} J_{x}<\infty .
$$

Esta hipótese é importante para evitar trivialidades já que os sistemas ferromagnéticos que não a satisfazem têm todos seus spins totalmente ordenados em qualquer temperatura finita [3].

A análise feita neste trabalho pode ser estendida para variáveis de spin na classe de Grifiths-Simon, nas quais suas distribuições podem ser reescritas a partir das chamadas variáveis de blocos de um sistema de spins de Ising ferromagnetico ou como um limite distribucional de tais variáveis, exemplos de tais spins variáveis são os spins de Ising e as variáveis da teoria quântica de campos $P\left(\phi^{4}\right)$.

Na demostração dos principais resultados do trabalho de AizenmanBarsky-Fernández [3] são usadas duas desigualdades diferenciais não lineares e também os resultados do artigo sobre resultado semelhante para o modelo de percolação [2]. Para obtenção de tais desigualdades são usadas as representações por correntes e passeios aleatórios que foram originalmente introduzidos no contexto de Teoria Quântica de Campos. Essas técnicas são descritas em detalhes no trabalho Geometric Analysis of $\phi^{4}$ Fields and Ising Models Parts I and II de Michael Ainzenman [1], tais ferramentas permitem explorar propriedades multiplicativas e combinatórias das funções de partição e de correlação. Sobre a representação em passeios aleatórios seguimos alguns passos do trabalho precursor desta técnica que é o artigo The Random-Walk Representation of Classical Spin Systems and Correlation Inequalities de Brydges, Frölich e Spencer de 1983 [11].

O artigo de Ainzenman, Barsky e Fernández [3] tem sido referência importante em centenas de artigos, e continua sendo, como por exemplo nos dois que seguem. 
No artigo do 2009 de J. E. Björberg e G. R. Grimett intitulado The Phase Transition of The Quantum Ising Model is Sharp, o spin quântico é descrito, e é desenvolvido um método similar ao de correntes aleatórios, mas para spins quânticos, esse método é chamado de representação por paridade aleatória. Valendo-se disso e utilizando procedimentos similares ao de [3] se deduz uma desigualdade para a magnetização

$$
M \leq \gamma \chi+M^{3}+2 \lambda M^{2} \frac{\partial M}{\partial \lambda}-2 \delta M^{2} \frac{\partial M}{\partial \delta},
$$

mas o objetivo é o análise da transição de fase no modelo de spins quânticos.

Também tem influenciado no artigo de 2014 de Ainzenman, Duminilcopin e Sidoravicius Random Current and Continuity of Ising Model's Spontaneous Magnetization [4], onde analisa-se a continuidade da magnetização no ponto crítico. Consegue-se provar que a magnetização espontânea desaparece para modelos de Ising ferromagnéticos em $\mathbb{Z}^{d}$. No [3] o Teorema 1 expressa resultados sobre a transição de fase, mas sobre pontos diferentes a $\beta=\beta_{c}$, com o artigo [4] temos solucionada essa dúvida no ponto crítico. 


\section{Apresentação}

O objetivo central deste texto é provar o Teorema 7.0.3. Para chegar neste resultado as principais ferramentas são os Teoremas 6.0.2 e 5.0.1. Este dois últimos teoremas são obtidos usando representações, da função de partição e de suas derivadas nas chamadas representações em Correntes Aleatórias e em Passeios Aleatórios. Tais representações permitirão o estudo de propriedades finas da magnetização e obtenção do comportamento assintótico desta função com respeito a alguns de seus parâmetros.

Dividimos em três partes os argumentos: Na primeira - Capítulo 1, 2 e 3 - apresentaremos a teoria básica que será usada nas partes seguintes. Definiremos o modelo de Ising e apresentaremos algumas de suas propriedades como a magnetização, susceptibilidade, as desigualdades de correlação, equações DLR, existência de Medidas de Gibbs e alguns conceitos sobre transição de fase. No Capítulo 1 assumimos que o leitor já conheça o modelo de Ising (pelo menos a volume finito) e fazemos uma revisão em forma de listagem das principais propriedades que vamos precisar a frente. No Capítulo 2, apresentaremos a classe de Griffiths-Simon, e todos os detalhes das provas das desigualdades GKS I e II e também da Desigualdade de Simon-Lieb. O Capítulo 3 trata de medidas de Gibbs a volume infinito. Mostramos a existência de tais medidas e apresentamos o conceito de transição de fase.

A segunda parte será dedicada à prova do Teorema 6.0.2. Iniciando-se no capítulo 4, onde definimos as representações por Correntes e Passeios Aleatórios e apresentamos suas principais propriedades.

Na sequência - Capítulo 5 - obteremos a primeira desigualdade diferencial (Teorema 5.0.1), que é válida para o modelo de Ising. A extensão desta desigualdade para modelos mais gerais pertencentes a classe Griffiths-Simon é obtida no Capítulo 6 no Teorema 5.0.1.

A terceira parte - Capítulo 7 - é dedicada à prova do Teorema 7.0.3. Usaremos as duas desigualdades, obtidas nos Capítulos 5 e 6 e alguns resultados clássicos de percolação para provar o Teorema 7.0.3. Como consequência deste teorema obtemos igualdade das temperaturas críticas para magnetização e susceptibilidade que é o resultado mais importante deste trabalho. 


\section{Sumário}

I Modelo de Ising e Mecânica Estatística 1

1 O Modelo de Ising 3

2 Classe de Griffiths-Simon $\quad 11$

2.1 Prova das Desigualdades GKS I e II . . . . . . . . . . . . . 14

2.2 Prova da Desigualdade de Simon-Lieb . . . . . . . . . . 18

3 Medidas de Gibbs a Volume Infinito e Transição de Fase 25

3.1 Medidas de probabilidade em $\Omega \ldots \ldots \ldots \ldots \ldots$

3.2 Especificações e Medidas . . . . . . . . . . . . . . . 33

3.3 Existência de Medidas em $\mathcal{G}(\pi)$. . . . . . . . . . . . . 42

\begin{tabular}{|ll}
\hline II Desigualdades Diferenciais & 47
\end{tabular}

$\begin{array}{|lll|}4 & \text { Representações por Correntes e Passeios aleatórios } & 49\end{array}$

4.1 Representações por Correntes Aleatórias . . . . . . . . . . . 49

5 Prova da Principal Desigualdade Diferencial 63

$\begin{array}{|lll|}6 & \text { Desigualdades Diferenciais na Classe de Griffiths-Simon }\end{array}$

III Igualdade das Temperaturas Críticas $\beta_{c}=\beta_{m} \quad \mathbf{8 1}$

\begin{tabular}{|lll}
7 & Consequências das Desigualdades Diferenciais 83
\end{tabular}

7.1 Prova dos Principais Resultados . . . . . . . . . . . . . 85

\begin{tabular}{ll}
\hline Referências Bibliográficas & 91
\end{tabular} 



\section{Parte I}

\section{Modelo de Ising e Mecânica Estatística}





\section{Capítulo 1}

\section{O Modelo de Ising}

Neste capítulo apresentamos em forma de uma lista as definições e propriedades básicas do modelo de Ising a volume finito. O objetivo é apenas fixar a notação que será usada por todo o restante do texto.

O modelo de Ising é um modelo matemático usado para entender o comportamento de alguns sistema físicos, fazendo o estudo de propriedades como magnetização, susceptibilidade magnética, pressão, energia livre, transição de fase, dentre outras.

Como em um sistema físico muitas variáveis são envolvidas, por exemplo, cada variável correspondendo a um átomo; para estudar uma propriedade como a magnetização do sistema do ponto de vista da Mecânica Clássica seria necessário considerar uma quantidade enorme de equações o que torna esta abordagem completamente inviável. A saida é estudar tais propriedades são estudadas estatisticamente através do uso de médias de cada uma das variáveis envolvidas. O modelo de Ising é o mais simples e mais importante de todos os modelos da Mecânica Estatística e é também um dos mais estudados. Este modelo foi criado por Wilhelm Lenz em 1920, e foi usado na tese de seu aluno de Doutorado Ernst Ising em 1925. Por causa dos resultados obtidos por Ernst Ising, este modelo foi chamado por Rudolph Peierls modelo de Ising.

Nossa apresentação/revisão do modelo de Ising segue de perto as referências [22], [9] e [21].

Inicialmente definiremos o modelo de Ising sobre um subgrafo finito $G_{\Lambda}=\left(\Lambda, \mathcal{E}_{\Lambda}\right)$ do grafo $\mathbb{L}^{d}=\left(\mathbb{Z}^{d}, \mathbb{E}^{d}\right)$ que tem como conjunto de vértices o reticulado inteiro $d$-dimensional $\mathbb{Z}^{d}$ e conjunto de arestas $\mathbb{E}^{d}$ dada pelos pares de vértices de primeiros vizinhos na norma $\ell_{1}$, isto é, $\{i, j\} \in \mathbb{E}^{d}$ se $\|i-j\|_{1}=1$, onde $\|i-j\|_{1}=\sum_{k=1}^{d}\left|i_{k}-j_{k}\right|$, embora possa ser definido sobre 
qualquer outro grafo.

Rede. Um subconjunto arbitrário $\Lambda$ de $\mathbb{Z}^{d}$ será às vezes chamado rede.

Observação 1.0.1. A menos que seja dito ao contrário, todas as definições apresentadas neste capítulo serão dadas a volume finito $(|\Lambda|<\infty)$.

Sítio. Cada ponto $i:=\left(i_{1}, i_{2}, \cdots, i_{d}\right)$ de $\Lambda$ será chamado sítio $i$.

Configuração. A aplicação

$$
\begin{aligned}
\omega: \mathbb{Z}^{d} & \rightarrow\{-1,1\} \\
i & \mapsto \omega(i)=: \omega_{i}
\end{aligned}
$$

será chamada de uma configuração. O conjunto de todas as configurações $\omega$ será chamado o espaço das configurações, e será denotado por $\Omega=$ $\{-1,1\}^{\mathbb{Z}^{d}}$. Note que $\left.\omega\right|_{\Lambda}$ a restrição de $\omega$ a $\Lambda \in \mathbb{Z}^{d}$ pode ser identificada com a família $\left(\omega_{i}\right)_{i \in \Lambda}$. O conjunto das configurações restritas a $\Lambda \in \mathbb{Z}^{d}$, será denotado por $\Omega_{\Lambda}$.

\section{Observação 1.0.2.}

$\triangleright$ Quando $\Lambda$ é finito $\Omega_{\Lambda}$ tem $2^{|\Lambda|}$ elementos.

$\triangleright$ Quando $\Lambda$ é infinito $\Omega_{\Lambda}$ é um conjunto discreto não enumerável.

Spin. A cada $i \in \mathbb{Z}^{d}$ associamos uma variável aleatória $\sigma_{i}$ definida no espaço mensurável $(\Omega, \mathcal{F})$, onde $\mathcal{F}$ é a $\sigma$-álgebra produto e

$$
\begin{aligned}
\sigma_{i}: \Omega & \rightarrow\{-1,1\} \\
\omega & \mapsto \sigma_{i}(\omega):=\omega_{i} .
\end{aligned}
$$

A variável aleatória $\sigma_{i}$ será chamada spin no sítio $i$.

Em analogia com notação introduzida acima, vamos usar a notação $\sigma=\left(\sigma_{i}\right)_{i \in \mathbb{Z}^{d}}$ e $\left.\sigma\right|_{\Lambda}=\left(\sigma_{i}\right)_{i \in \Lambda}$. Deve se ter em mente que em várias situações vamos usar a notação $\sigma$ também para denotar um elemento genérico de $\Omega$ ou $\Omega_{\Lambda}$ e nestes casos $\sigma_{i}$ significa o valor da $i$-ésima coordenada da configuração $\sigma$. Apesar desta aparente confusão será sempre claro pelo contexto se $\sigma_{i}$ denota uma variável aleatória ou a $i$-ésima coordenada de uma configuração $\sigma \in \Omega$. Por exemplo, se $\omega, \sigma$ são elementos arbitrários de $\Omega$, então a v.a. $\sigma_{i}$ calculada nestes pontos é dada respectivamente por $\sigma_{i}(\omega)=\omega_{i}$ e $\sigma_{i}(\sigma)=\sigma_{i}$. Claramente $\sigma_{i}$ no lado direito da última igualdade significa a $i$-ésima coordenada da configuração $\sigma$ e não a v.a. $\sigma_{i}$. Por razões óbvias 
vamos evitar escrever isto. Por outro lado, se $\mu$ é uma medida de probabilidade em $(\Omega, \mathcal{F})$ as notações $\mathbb{E}_{\mu}\left[\sigma_{i}\right], \mu\left(\sigma_{i}\right)$ ou $\int_{\Omega} \sigma_{i} d \mu$ se referem claramente a esperança da variável $\sigma_{i}$ com respeito a medida de probabilidade $\mu$.

Vamos nos referir ao evento $\left\{\sigma_{i}=+1\right\}$ dizendo que o spin no sítio $i$ vale +1 ou então que ele está orientado para cima. Por outro lado, quando ocorrer o evento $\left\{\sigma_{i}=-1\right\}$ vamos dizer que o spin no sítio $i$ vale -1 ou que o spin neste sítio está orientado para baixo.

Condições de contorno. Vamos usar os elementos de $\Omega$ também para definir condições de contorno (ou exteriores) da seguinte forma. Fixado $\bar{\omega} \in \Omega$, definimos

$$
\Omega_{\Lambda}^{\bar{\omega}}:=\left\{\omega \in \Omega: \omega_{i}=\bar{\omega}_{i}, \forall i \notin \Lambda\right\} .
$$

Neste contexto chamamos $\bar{\omega}$ de uma condição de contorno. Observe que $\bar{\omega}$ é uma configuração fixa que serve para definir a partir de uma configuração $\sigma \in \Omega_{\Lambda}$ uma nova configuração $\omega$ em $\Omega$ tal que valores de $\left.\omega\right|_{\Lambda}=\sigma$ e $\left.\omega\right|_{\Lambda^{c}}=\left.\bar{\omega}\right|_{\Lambda^{c}}$.

No conjunto de todas as configurações algumas delas se destacam, é o caso das condições de contorno que satisfazem

- $\bar{\omega}_{i} \equiv+1, \forall i \in \mathbb{Z}^{d}$, chamada de condição de contorno +;

- $\bar{\omega}_{i} \equiv-1, \forall i \in \mathbb{Z}^{d}$, chamada de condição de contorno -.

Para o cálculo de algumas funções termodinâmicas que não dependem da condição de contorno é conveniente introduzir a chamada condição de contorno livre, esta não é obtida pela definição acima e falaremos dela no momento adequado. É importante também mencionar que muitos cálculos são facilitados quando estamos trabalhando no toro discreto $d$-dimensional de comprimento $N$, isto é na rede dada pelo espaço quociente $\mathbb{Z} / N \mathbb{Z} \times \ldots \times \mathbb{Z} / N \mathbb{Z}$. A passagem da rede $\Lambda$ para um toro deste tipo pode ser feita usando a chamada condição de contorno periódica . Neste caso a rede $\Lambda$ é substituída por uma rede em que o conjunto de sítios é dado por $\Lambda=\{1, \ldots, N+1\}^{d}$ e as arestas são dadas por pares de sítios vizinhos $(\|i-j\|=1)$ e também por pare de sítios $i, j$ (desta vez não precisam ser vizinhos) tais que existe $k \in\{1, \ldots, d\}$ para o qual $i_{k}-j_{k} \equiv 0(\bmod N)$, veja figura 1.1 . 

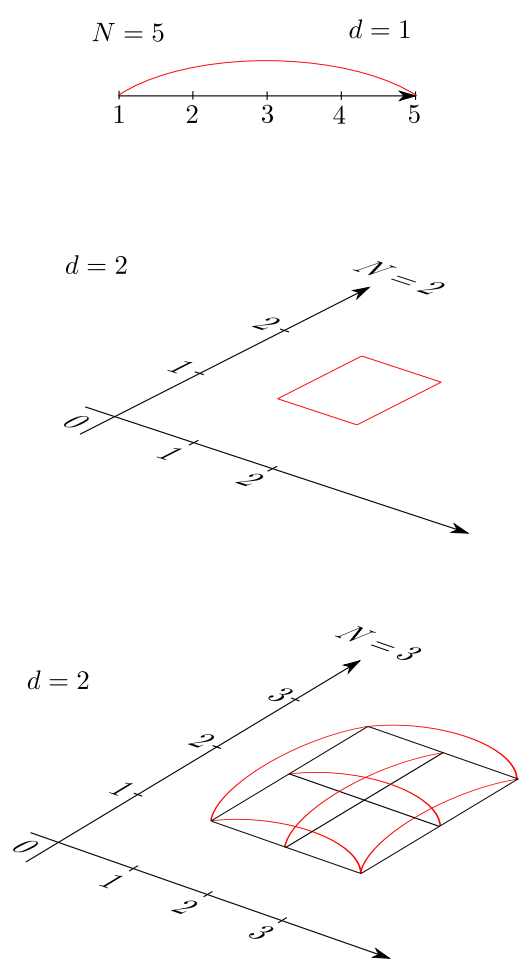

Figura 1.1: Representação de toros em dimensão 1 e 2.

Constantes de Acoplamento. Para cada par de sítios distintos $i, j \in \Lambda$ vamos considerar constantes $J_{i j}$. No modelo de Ising estas constantes são interpretadas como energia de interação entre spins $\sigma_{i}$ e $\sigma_{j}$. Quando estas constantes são escolhidas de forma que $J_{i j} \geq 0$, dizemos que o modelo de Ising com tal acoplamento é um modelo ferromagnético, e quando $J_{i j} \leq 0$, que o modelo é antiferromagnético.

Falamos que temos invariância por translações no sistema, quando se satisfaz que $J_{x, y}=J_{0, x-y}$

Hamiltoniano do Modelo de Ising: A energia associada a cada configuração $\sigma \in \Omega$ no volume $\Lambda \subset \mathbb{Z}^{d}$, com constantes de acoplamento $J_{i j} \geq 0$ (trabalharemos com modelos ferromagnéticos), campo magnético externo $h_{i} \in \mathbb{R}$ e condições de contorno $\bar{\omega}$ é dada pelo número real

$$
H_{\Lambda, h}^{\bar{\omega}}(\sigma)=-\sum_{\{i, j\} \in \mathcal{E}_{\Lambda}} J_{i j} \sigma_{i}(\omega) \sigma_{j}(\omega)-\sum_{i \in \Lambda} h_{i} \sigma_{i}-\sum_{\{i, j\}: i \in \Lambda, j \in \partial \Lambda} J_{i j} \sigma_{i}(\omega) \bar{\omega}_{j},
$$

onde $\partial \Lambda$ é a fronteira externa de $\Lambda$,i.e., o conjunto de sítios $i \notin \Lambda$ tais que tem distância 1 a $\Lambda$, e $\{i, j\}$ são pares vizinhos $(\|i-j\|=1$ ). A primeira soma descreve a energia das interações de dois corpos e é realizada sobre todos os pares de primeiros vizinhos, a segunda soma descreve as interações dos spins de $\Lambda$ com o campo externo $h_{i}$ em cada sítio $i \in \Lambda$ e a terceira soma 
descreve a interações do sistema com o exterior de $\Lambda$ fixado no estado $\bar{\omega}$. Nos referimos a aplicação $\omega \mapsto H_{\Lambda, h}^{\bar{\omega}}(\omega)$ como o Hamiltoniano do modelo de Ising no volume $\Lambda$ com condição de contorno $\bar{\omega}$.

Função de partição. É uma constante de normalização constituída pela soma dos chamados fatores de Boltzmann $\left(e^{-\beta H_{\Lambda, h}^{\bar{\omega}}(\omega)}\right)$ sobre todas as configurações $\omega \in \Omega_{\Lambda}$, e sua expressão é dada por

$$
Z_{\Lambda, \beta, h}^{\bar{\omega}}:=\sum_{\omega \in \Omega_{\Lambda}^{\bar{\omega}}} e^{-\beta H_{\Lambda, h}^{\bar{\Phi}}(\omega)}
$$

onde $\beta=\frac{1}{k T}$, $T$ é a temperatura e $k$ é a constante de Boltzmann (por simplicidade vamos assumir neste trabalho que $k=1$ ).

Energia Livre (volume finito). A quantidade

$$
f_{\Lambda}^{\bar{\omega}}(\beta, h):=-(\beta|\Lambda|)^{-1} \log \left(Z_{\Lambda, \beta, h}^{\bar{\omega}}\right)
$$

será chamada de energia livre a volume finito, e a quantidade $p_{\Lambda}^{\bar{\omega}}(\beta, h):=$ $|\Lambda|^{-1} \log \left(Z_{\Lambda, \beta, h}^{\bar{\omega}}\right)$ será chamada de pressão a volume finito.

Medida de Gibbs (volume finito). A medida de probabilidade que associada a cada configuração $\omega \in \Omega_{\Lambda}^{\bar{\omega}}$, a probabilidade

$$
\mu_{\Lambda, \beta, h}^{\bar{\omega}}(\omega):=\frac{e^{-\beta H_{\Lambda, h}^{\bar{\omega}}(\omega)}}{Z_{\Lambda, \beta, h}^{\bar{\omega}}}
$$

é chamada de medida de Gibbs do modelo de Ising a volume $\Lambda$ com condições de contorno $\bar{\omega}$.

Medida de Gibbs (volume finito) com condições de Contorno Livre. A medida de Gibbs do modelo de Ising com condições de contorno livre é definida por

$$
\mu_{\Lambda, \beta, h}^{\emptyset}(\omega):=\frac{1}{Z_{\Lambda, \beta, h}^{\emptyset}} \exp \left(-\beta\left\{-\sum_{\{i, j\} \in \mathcal{E}_{\Lambda}} J_{i j} \sigma_{i}(\omega) \sigma_{j}(\omega)-\sum_{i \in \Lambda} h_{i} \sigma_{i}(\omega)\right\}\right) .
$$

a função $\omega \mapsto H_{\Lambda, h}^{\emptyset}(\omega)$ dada por

$$
H_{\Lambda, h}^{\emptyset}(\omega):=-\sum_{\{i, j\} \in \mathcal{E}_{\Lambda}} J_{i j} \sigma_{i}(\omega) \sigma_{j}(\omega)-\sum_{i \in \Lambda} h_{i} \sigma_{i}(\omega)
$$

será chamada de Hamiltoniano no modelo de Ising a volume $\Lambda$ com con- 
dições de contorno livre.

Valor médio. O valor médio de uma função $\phi: \Omega \rightarrow \mathbb{R}$, com respeito a medida de Gibbs $\mu_{\Lambda, \beta, h}^{\bar{\omega}}$, é definido como sendo a integral de Lebesgue de $\phi$ com respeito a medida $\mu_{\Lambda, \beta, h}^{\bar{\omega}}$ e será denotado por

$$
\langle\phi\rangle_{\Lambda, \beta, h}^{\bar{\omega}}:=\frac{1}{Z_{\Lambda, \beta, h}^{\bar{\omega}}} \sum_{\omega \in \Omega_{\Lambda}^{\bar{\omega}}} \phi(\omega) e^{-\beta H_{\Lambda, h}^{\bar{\omega}}(\omega)},
$$

- se $\phi(\omega)=\prod_{k=1}^{n} \sigma_{i_{k}}(\omega)$, então seu valor médio $\langle\phi\rangle_{\Lambda, \beta, h}^{\bar{\omega}}$ é chamado de função de correlação dos $n$ spins.

- se $\phi(\omega)=\sigma_{i}(\omega)$ então o valor médio $\langle\phi\rangle_{\Lambda, \beta, h}^{\bar{\omega}_{n}}$ é chamado de magnetização do sítio $i$.

- se $\phi(\omega)=\sigma_{i}(\omega) \sigma_{j}(\omega)$ então chamamos a $\langle\phi\rangle_{\Lambda, \beta, h}^{\bar{\omega}}$ de correlação spin-spin.

Magnetização. A magnetização no modelo de Ising é definida pela seguinte expressão

$$
M_{\Lambda}^{\bar{\omega}}(\beta, h)=\frac{1}{|\Lambda|} \sum_{i \in \Lambda}\left\langle\sigma_{i}\right\rangle_{\Lambda, \beta, h}^{\bar{\omega}} .
$$

Como veremos mais a frente será de grande interesse considerar o limite da expressão acima quando $\Lambda \uparrow \mathbb{Z}^{d}$. Este limite pode ser considerado de várias maneiras. Podemos defini-lo considerando uma coleção arbitrária $\Lambda_{n} \subset \mathbb{Z}^{d}$ tal que $\cup_{n \in \mathbb{N}} \Lambda_{n}=\mathbb{Z}^{d}$ ou de maneira mais conveniente considerar simplesmente que o limite será tomado ao longo de hipercubos da forma $\Lambda_{n}=[-n, n]^{d} \cap \mathbb{Z}^{d}$. Neste caso, por exemplo, a expressão $\lim _{\Lambda \nearrow \mathbb{Z}^{d}} M_{\Lambda}^{\bar{\omega}}(\beta, h)$ tem o seguinte significado

$$
\lim _{\Lambda \succ \mathbb{Z}^{d}} M_{\Lambda}^{\bar{\omega}}(\beta, h):=\lim _{n \rightarrow \infty} M_{\Lambda_{n}}^{\bar{\omega}}(\beta, h)
$$

Mais a frente como uma aplicação das desigualdades GKS-II vamos mostrar que para as condições de contorno mais, menos e livre, isto é, $\bar{\omega}=+,-, \emptyset$ existe para todo $\beta>0$ e $h \in \mathbb{R}$ o seguinte limite

$$
M(\beta, h)=\lim _{\Lambda \nearrow \mathbb{Z}^{d}} M_{\Lambda}^{\bar{\omega}}(\beta, h) .
$$


Magnetização espontânea. Também conhecida como magnetização residual é definida pela expressão

$$
M^{\bar{\omega}}(\beta)=\lim _{h \rightarrow 0^{+}} M^{\bar{\omega}}(\beta, h) .
$$

Temos aqui novamente uma definição condicionada a existência de dois limites. O limite quando $\Lambda \nearrow \mathbb{Z}^{d}$ (que é comumente chamado de limite termodinâmico) é o mais delicado deles. É fácil mostrar que ele pode não existir para escolhas especiais da condição de contorno $\bar{\omega}$. Porém o limite quando $h \rightarrow 0^{+}$existe sempre que $J_{i j} \geq 0$, este fato pode ser demonstrado como uma consequência da Desigualdade de GKS-II.

Susceptibilidade magnética. A susceptibilidade magnética a volume $\Lambda$ com condições de contorno $\bar{\omega}$ é definida por

$$
\chi_{\Lambda}^{\bar{\omega}}(\beta, h)=\frac{1}{\beta} \frac{\partial}{\partial h} M_{\Lambda}^{\bar{\omega}}(\beta, h) .
$$

Vamos mostrar também como consequência da Desigualdade de GKS-II que se $J_{i j} \geq 0$ e $\bar{\omega}=+,-, \emptyset$ então existe o seguinte limite

$$
\chi^{\bar{\omega}}(\beta, h)=\lim _{\Lambda \nearrow \mathbb{Z}^{d}} \chi_{\Lambda}^{\bar{\omega}}(\beta, h),
$$

este limite será chamado de limite termodinâmico da susceptibilidade ou simplesmente a susceptibilidade a volume infinito.

Observação 1.0.3. Para não carregar a notação em certos pontos do texto vamos omitir a dependência de certas funções com respeito aos parâmetros $\beta, h_{i}, J_{i j}$. Serão carregados na notação, em cada seção, aqueles parâmetros que forem mais importantes para os fatos que estiverem sendo estabelecidos no momento. 
Capítulo 1. O Modelo de Ising 


\section{Capítulo 2}

\section{Classe de Griffiths-Simon}

Neste capítulo, apresentamos a classe de Griffiths-Simon. Será mostrado também como reescrever o modelo de Ising usando uma medida a priori no espaço $\{-1,1\}$ e vamos demonstrar também as principais desigualdades de correlação usadas neste trabalho que são as Desigualdades GKS-I, GKS-II e Simon-Lieb. Um grande número de aplicações das Desigualdade GKS I e II pode ser encontrado em [9], [10] e [21]. Para os demais conteúdos desta seção as principais referências são [19], [20], [7] e [13].

Observação 2.0.4. A seguir, simplificaremos algumas notações:

$$
\begin{aligned}
\langle\cdot\rangle_{\Lambda, \beta, h}^{\bar{\omega}} \equiv\langle\cdot\rangle_{\Lambda}, & Z_{\Lambda, \beta, h}^{\bar{\omega}} \equiv Z_{\Lambda}, \\
H_{\Lambda, h}^{\bar{\omega}}(\omega) \equiv H_{\Lambda}, & \sigma_{A}(\omega) \equiv \sigma_{A} .
\end{aligned}
$$

É importante enfatizar que só faremos simplificações quando não apresentar inconvenientes. Já que $\beta$ e h são parâmetros de grande relevância para vários estudos do sistema como, por exemplo, da transição de fase eles podem voltar à notação em momentos oportunos, como subíndices ou argumento de função.

No capítulo anterior definimos o modelo de Ising em uma rede $\Lambda$. Como foi mencionado este modelo é um exemplo de um modelo de Mecânica Estatística. No caso geral, um modelo de Mecânica Estatística a volume finito pode ser visto como um processo estocástico $\left\{X_{t}\right\}_{t \in T}$, onde o conjunto de índices $T=\Lambda \subset \mathbb{Z}^{d}$ e cuja distribuição conjunta em $\mathbb{R}^{\Lambda}$ tem a forma

$$
\frac{1}{Z_{\Lambda}} \exp \left(-\beta H_{\Lambda}(\sigma)\right) \prod_{i \in \Lambda} d \rho_{i}\left(\sigma_{i}\right)
$$


com $H_{\Lambda}$ sendo uma função de $\mathbb{R}^{\Lambda}$ em $\mathbb{R}$ e

$$
Z_{\Lambda}=\int_{\mathbb{R}^{\Lambda}} \exp \left(-\beta H_{\Lambda}(\sigma)\right) \prod_{i \in \Lambda} d \rho_{i}\left(\sigma_{i}\right) .
$$

As medidas $\rho_{i}$ que aparecem nesta definição são chamadas de medidas a priori no sítio $i$. Para evitar trivialidades vamos supor que as medidas a priori são escolhidas de forma que $Z_{\Lambda}<+\infty$.

Na maioria dos casos temos que para todo $i \in \mathbb{Z}$ a medida a priori $\rho_{i}=\rho$, ou seja, em todos os sítios da rede a medida a priori é a mesma.

Medida a priori na classe de Griffiths Simon. Uma medida a priori $\rho: \mathscr{B}(\mathbb{R}) \rightarrow[0,+\infty]$ está na classe de Griffiths-Simon (classe GS) se:

i) $\rho$ é par, isto é, $\rho$ é uma medida invariante pela aplicação $r(x)=-x$.

ii) A medida de Gibbs $\mu_{\Lambda}$, construída a partir da medida a priori $\rho$, com condições de contorno livre, satisfazem as Desigualdades de GKS-I, GKS-II e Simon-Lieb.

(Os enunciados das desigualdades mencionadas acima são apresentados no final desta seção).

Para ter em mente uma descrição mais simples de um modelo de Mecânica Estatística definido com medidas a priori vamos reescrever o modelo de Ising nesta forma.

Exemplo 2.0.1. O modelo de Ising escrito com sua medida a priori.

Para simplificar vamos considerar condições de contorno livre e denotar o Hamiltoniano $H_{\Lambda, h}^{\emptyset}$ simplesmente por $H_{\Lambda}$, os demais casos são tratados de maneira completamente análoga.

Considere a seguinte medida a priori

$$
\rho=\frac{1}{2} \delta_{+}-\frac{1}{2} \delta_{-},
$$

onde $\delta_{x}$ é a medida delta de Dirac suportada no ponto $x\left(\delta_{ \pm}:=\delta_{ \pm 1}\right)$. Se $\mu_{\Lambda}$ denota a medida do modelo de Ising com condições de fronteira livre, vamos mostrar que para todo $E \subset \Omega_{\Lambda}$ é válida a seguinte identidade:

$$
\mu_{\Lambda}(E)=\frac{\int_{\Omega_{\Lambda}} 1_{E}(\omega) e^{-\beta H_{\Lambda}(\omega)} \prod_{i \in \Lambda} d \rho\left(\omega_{i}\right)}{\int_{\Omega_{\Lambda}} e^{-\beta H_{\Lambda}(\omega)} \prod_{i \in \Lambda} d \rho\left(\omega_{i}\right) .}
$$


Primeiro observe que $\rho\left(\left\{\omega_{i}=1\right\}\right)=\rho\left(\left\{\omega_{i}=-1\right\}\right)=\frac{1}{2}$, para todo $i \in \Lambda$. Logo temos que

$$
\prod_{i \in \Lambda} d \rho\left(\omega_{i}\right)=\frac{1}{2^{|\Lambda|}} d \vartheta(\omega),
$$

onde $\vartheta$ é a medida de contagem em $\Omega_{\Lambda}$. Desta forma podemos reescrever o lado direito de (2.1) como segue

$$
\frac{\int_{\Omega_{\Lambda}} 1_{E}(\omega) e^{-\beta H_{\Lambda}} \frac{1}{2^{|\Lambda|}} \vartheta(d \omega)}{\int_{\Omega_{\Lambda}} e^{-\beta H_{\Lambda}} \frac{1}{2^{|\Lambda|}} \vartheta(d \omega)} .
$$

Já que $\Omega_{\Lambda}$ é um conjunto discreto e $\vartheta$ é uma medida de contagem, segue que as integrais acima são dadas pelos seguintes somatórios:

$$
\frac{\sum_{\omega \in \Omega_{\Lambda}} 1_{E}(\omega) e^{-\beta H_{\Lambda}(\omega)}}{\sum_{\omega \in \Omega_{\Lambda}} e^{-\beta H_{\Lambda}(\omega)}} .
$$

Tomando $E=\{\omega\}$ temos imediatamente (1.3) $\operatorname{com} \bar{\omega}=\emptyset$, ou seja,

$$
\mu_{\Lambda}(\{\omega\})=\frac{e^{-\beta H_{\Lambda}(\omega)}}{\sum_{\omega^{\prime} \in \Omega_{\Lambda}} e^{-\beta H_{\Lambda}\left(\omega^{\prime}\right)}} .
$$

No que segue, apresentaremos as Desigualdades GKS I e II e SimonLieb, seus enunciados e suas respectivas demonstrações. Antes porém precisamos de mais uma definição.

- Sejam $\Lambda \subset \mathbb{Z}^{d}$ e $A \subset \Lambda$ finito. Definimos a variável aleatória $\sigma_{A}: \Omega_{\Lambda} \rightarrow \mathbb{R}$ por $\sigma_{A}:=\prod_{i \in A} \sigma_{i}$.

Teorema 2.0.1 (Desigualdade GKS). Para o modelo de Ising d-dimensional com Hamiltoniano (1.1) e com condições de contorno positivas, livre, periódicas ou combinação destas temos

GKS-I) para todo $A \subset \Lambda \boldsymbol{e} \beta, h>0$,

$$
\left\langle\sigma_{A}\right\rangle_{\Lambda, \beta, h}^{\bar{\omega}} \geq 0
$$

GKS-I) para qualquer $A, B \subset \Lambda, \boldsymbol{e} \beta, h \geq 0$,

$$
\left\langle\sigma_{A} \sigma_{B}\right\rangle_{\Lambda, \beta, h}^{\bar{\omega}} \geq\left\langle\sigma_{A}\right\rangle_{\Lambda, \beta, h}^{\bar{\omega}}\left\langle\sigma_{B}\right\rangle_{\Lambda, \beta, h}^{\bar{\omega}} .
$$


Teorema 2.0.2 (Desigualdade de Simon-Lieb). Dado o modelo de Ising $d$-dimensional com interação de primeiros vizinhos e com Hamiltoniano dado por (1.1), com $h_{i}=0$ e com condições de contorno livres. Sejam $x e$ y sítios quaisquer de uma rede finita $\Lambda \subset \mathbb{Z}^{d}$ e seja $\Gamma$ um subconjunto finito de $\mathbb{Z}^{d}$, cuja fronteira $\partial \Gamma$ separa $x$ e $y$, isto é, todo caminho na rede $\Lambda$ ligando $x$ a y necessariamente passa por um vértice de $\Gamma$. Então

$$
\left\langle\sigma_{x} \sigma_{y}\right\rangle_{\Lambda} \leq \sum_{d \in \partial \Gamma}\left\langle\sigma_{x} \sigma_{d}\right\rangle_{\Gamma}\left\langle\sigma_{d} \sigma_{y}\right\rangle_{\Lambda}
$$

\subsection{Prova das Desigualdades GKS I e II}

Demonstração de GKS I. Apresentaremos a prova para condições de fronteira livre. Como o denominador da esperançá é $Z_{\Lambda}>0$, so temos que provar que o numerador é não negativo ou seja,

$$
\sum_{\omega \in \Omega_{\Lambda}} \sigma_{A}(\omega) e^{-\beta H_{\Lambda}(\omega)} \geq 0
$$

com esse fim trabalhamos no fator de Boltzmann

$$
e^{-\beta H_{\Lambda}(\omega)}=\prod_{i \in \Lambda} e^{\beta h_{i} \omega_{i}} \prod_{\{i, j\} \in \mathcal{E}_{\Lambda}} e^{\beta J_{i j} \omega_{i} \omega_{j}}
$$

e, como

$$
e^{\beta J_{i j} \omega_{i} \omega_{j}}=\sum_{n=0}^{\infty} \frac{\left(\beta J_{i j} \omega_{i} \omega_{j}\right)^{n}}{n !}
$$

podemos ordenar os termos de forma a obter as seguintes igualdades

$$
\begin{aligned}
e^{\beta J_{i j} \omega_{i} \omega_{j}} & =\cosh \left(\beta J_{i j}\right)+\operatorname{senh}\left(\beta J_{i j}\right) \omega_{i} \omega_{j} \\
& =\cosh \left(\beta J_{i j}\right)\left(1+\tanh \left(\beta J_{i j}\right) \omega_{i} \omega_{j}\right) .
\end{aligned}
$$

Agora usando (2.6) em (2.5) temos

$$
e^{-\beta H_{\Lambda}(\omega)}=\prod_{i \in \Lambda} e^{\beta h_{i} \omega_{i}} \prod_{\{i, j\} \in \mathcal{E}_{\Lambda}} \cosh \left(\beta J_{i j}\right)\left(1+\tanh \left(\beta J_{i j}\right) \omega_{i} \omega_{j}\right),
$$

logo,

$$
\sum_{\omega \in \Omega_{\Lambda}} \sigma_{A}(\omega) e^{-\beta H_{\Lambda}(\omega)}=\sum_{\omega \in \Omega_{\Lambda}} \prod_{i \in \Lambda} e^{\beta h_{i} \omega_{i}} \prod_{\{i, j\} \in \mathcal{E}_{\Lambda}} \cosh \left(\beta J_{i j}\right)\left(\sigma_{A}(\omega)+\tanh \left(\beta J_{i j}\right) \omega_{i} \omega_{j} \sigma_{A}(\omega)\right) .
$$


Agora desenvolvendo o produtório obtemos

$$
\prod_{i \in \Lambda} e^{\beta h_{i} \omega_{i}} \prod_{\{i, j\} \in \mathcal{E}_{\Lambda}} \cosh \left(\beta J_{i j}\right)\left(\sigma_{A}(\omega)+\tanh \left(\beta J_{i j}\right) \omega_{i} \omega_{j} \sigma_{A}(\omega)\right)=\sum_{m} c_{m} P_{m},
$$

onde $C_{m}$ é um fator que é um dado por um produto de termos que são ora $\cosh \left(\beta J_{i j}\right)$, ora $\operatorname{senh}\left(\beta J_{i j}\right)$, esses fatores são não-negativos pois $\cosh (x) \mathrm{e}$ $\tanh (x)$ são ambos não-negativos para $x \geq 0$. Já os fatores $P_{m}$ são da forma

$$
P_{m}=\omega_{i_{1}}^{n_{i_{1}}} \cdots \omega_{i_{m}}^{n_{i_{m}}} \prod_{i \in \Lambda} e^{\beta h_{i} \omega_{i}}
$$

com isto temos que

$$
\sum_{\omega \in \Omega_{\Lambda}} \sigma_{A}(\omega) e^{-\beta H_{\Lambda}(\omega)}=\sum_{\omega \in \Omega_{\Lambda}} \sum_{m} c_{m} P_{m}=\left(\sum_{m} c_{m}\right)\left(\sum_{\omega \in \Omega_{\Lambda}} P_{m}\right) .
$$

Caso todos os $n_{i}$ sejam pares temos imediatamente que

$$
\sum_{\omega \in \Omega_{\Lambda}} P_{m}=\sum_{\omega \in \Omega_{\Lambda}} \prod_{i \in \Lambda} e^{\beta h_{i} \omega_{i}}>0 .
$$

Por outro lado, se $n_{j_{1}}, \ldots, n_{j_{r}}$ é a lista completa de todos os expoentes ímpares que aparecem em $P_{m}$ então temos que

$$
P_{m}=\omega_{j_{1}} \ldots \omega_{j_{r}} \prod_{i \in \Lambda} e^{\beta h_{i} \omega_{i}} .
$$

Somando sobre todas as configurações ficamos com

$$
\sum_{\omega \in \Omega_{\Lambda}} P_{m}=\sum_{\omega \in \Omega_{\Lambda}} \omega_{j_{1}} \ldots \omega_{j_{r}} \prod_{i \in\left\{j_{1}, \ldots, j_{r}\right\}} e^{\beta h_{i} \omega_{i}} \prod_{i \in \Lambda \backslash\left\{j_{1}, \ldots, j_{r}\right\}} e^{\beta h_{i} \omega_{i}}
$$

como o segundo produtório que aparece acima é positivo e

$$
e^{\beta h_{j}}-e^{-\beta h_{j}}=2 \operatorname{senh}\left(\beta h_{j}\right) \geq 0,
$$

segue que

$$
\sum_{\omega \in \Omega_{\Lambda}} P_{m} \geq 0
$$


Assim concluímos que

$$
\sum_{\omega \in \Omega_{\Lambda}} \sigma_{A}(\omega) e^{-\beta H_{\Lambda}(\omega)} \geq 0
$$

Demonstração de GKS II. Faremos uma prova para o modelo em condições de fronteira livre. O método utilizado nesta prova será o de duplicação de variáveis, ou seja, para cada sitio $i \in \Lambda$ criamos uma nova variável $\mu_{i}: \Omega_{\Lambda} \rightarrow\{-1,1\}$, e e definimos um Hamiltoniano duplicado no volume $\Lambda$ da seguinte forma

$$
H^{(2)}(\sigma, \mu):=H(\sigma)+H(\mu)=-\sum_{\{i, j\} \in \mathcal{E}_{\Lambda}} J_{i, j}\left(\sigma_{i} \sigma_{j}+\mu_{i} \mu_{j}\right)-\sum_{i \in \Lambda} h_{i}\left(\sigma_{i}+\mu_{i}\right) .
$$

Denotaremos por $\langle\cdot\rangle^{(2)}$ o valor esperado com respeito a medida de Gibbs definida pelo o hamiltoniano $H^{(2)}(\sigma, \mu)$. Observe que

$$
\begin{aligned}
\left\langle\sigma_{A} \sigma_{B}-\sigma_{A} \mu_{B}\right\rangle^{(2)}= & \frac{\sum_{\omega_{1} \in \Omega_{\Lambda}} \omega_{\omega_{2} \in \Omega_{\Lambda}}\left[\sigma_{A}\left(\omega_{1}\right) \sigma_{B}\left(\omega_{1}\right)-\sigma_{A}\left(\omega_{1}\right) \mu_{B}\left(\omega_{2}\right)\right] e^{-\beta(H(\sigma))\left(\omega_{1}\right)-\beta(H(\mu))\left(\omega_{2}\right)}}{\sum_{\omega_{1} \in \Omega_{\Lambda}} \sum_{\omega_{2} \in \Omega_{\Lambda}} e^{-\beta(H(\sigma))\left(\omega_{1}\right)-\beta(H(\mu))\left(\omega_{2}\right)}} \\
= & \frac{\sum_{\omega_{1} \in \Omega_{\Lambda}} \sigma_{A}\left(\omega_{1}\right) \sigma_{B}\left(\omega_{1}\right) e^{-\beta(H(\sigma))\left(\omega_{1}\right)} \sum_{\omega_{2} \in \Omega_{\Lambda}} e^{-\beta(H(\mu))\left(\omega_{2}\right)}}{\sum_{\omega_{2} \in \Omega_{\Lambda}} e^{-\beta(H(\mu))\left(\omega_{2}\right)} \sum_{\omega_{1} \in \Omega_{\Lambda}} e^{-\beta(H(\sigma))\left(\omega_{1}\right)}} \\
& -\frac{\sum_{\omega_{2} \in \Omega_{\Lambda}} \mu_{B}\left(\omega_{2}\right) e^{-\beta(H(\mu))\left(\omega_{2}\right)} \sum_{\omega_{1} \in \Omega_{\Lambda}} \sigma_{A}\left(\omega_{1}\right) e^{-\beta(H(\sigma))\left(\omega_{1}\right)}}{\sum_{\omega_{2} \in \Omega_{\Lambda}} e^{-\beta(H(\mu))\left(\omega_{2}\right)} \sum_{\omega_{1} \in \Omega_{\Lambda}} e^{-\beta(H(\sigma))\left(\omega_{1}\right)}} \\
= & \left\langle\sigma_{A} \sigma_{B}\right\rangle-\left\langle\sigma_{B}\right\rangle\left\langle\sigma_{A}\right\rangle
\end{aligned}
$$

e da mesma forma temos as seguintes igualdades

$$
\begin{gathered}
\left\langle\sigma_{A}\right\rangle^{(2)}=\left\langle\mu_{A}\right\rangle^{(2)}=\left\langle\sigma_{A}\right\rangle, \\
\left\langle\sigma_{A} \mu_{B}\right\rangle^{(2)}=\left\langle\sigma_{A}\right\rangle\left\langle\mu_{B}\right\rangle, \\
\left\langle\sigma_{A} \sigma_{B}\right\rangle^{(2)}=\left\langle\sigma_{A} \sigma_{B}\right\rangle=\left\langle\mu_{A} \mu_{B}\right\rangle=\left\langle\mu_{A} \mu_{B}\right\rangle^{(2)} .
\end{gathered}
$$


Como $\sigma_{A}=\prod_{i \in A} \sigma_{i}$, temos

$$
\left\langle\sigma_{A} \sigma_{B}-\sigma_{A} \mu_{B}\right\rangle^{(2)}=\left\langle\prod_{i \in A} \sigma_{i}\left(\prod_{i \in B} \sigma_{i}-\prod_{i \in B} \mu_{i}\right)\right\rangle^{(2)} .
$$

Considerando a seguinte mudança de variáveis

$$
X_{i}=\frac{\left(\sigma_{i}-\mu_{i}\right)}{\sqrt{2}} \text { e } \quad Y_{i}=\frac{\left(\sigma_{i}+\mu_{i}\right)}{\sqrt{2}}
$$

e reescrevendo as igualdades em (2.8) com (2.9), ficamos com

$$
\begin{gathered}
\left\langle\sigma_{A} \sigma_{B}\right\rangle-\left\langle\sigma_{B}\right\rangle\left\langle\sigma_{A}\right\rangle=\left\langle\sigma_{A} \sigma_{B}-\sigma_{A} \mu_{B}\right\rangle^{(2)} \\
=\left\langle\prod_{i \in A}\left(\frac{X_{i}+Y_{i}}{\sqrt{2}}\right)\left[\prod_{i \in B}\left(\frac{X_{i}+Y_{i}}{\sqrt{2}}\right)-\prod_{i \in B}\left(\frac{-X_{i}+Y_{i}}{\sqrt{2}}\right)\right]\right\rangle^{(2)} .
\end{gathered}
$$

Os termos com coeficiente negativo de $\prod_{i \in B}\left(\frac{-X_{i}+Y_{i}}{\sqrt{2}}\right)$ são cancelados com o termo de $\prod_{i \in B}\left(\frac{X_{i}+Y_{i}}{\sqrt{2}}\right)$ e ao final fica um polinômio em $X_{i}$ e $Y_{i}$ com coeficientes não negativos. A demostração fica terminada se mostramos que $\left\langle X_{A} Y_{B}\right\rangle^{(2)} \geq 0$, para quaisquer $A, B \subset \Lambda$, onde $X_{A}:=\prod_{i \in A} X_{i}$. Para provar esta desigualdade primeiro observamos que

$$
\begin{gathered}
H^{(2)}(\sigma, \mu)=-\sum_{\{i, j\} \in \mathcal{E}_{\Lambda}} J_{i, j}\left(X_{i} X_{j}+Y_{i} Y_{j}\right)-\sqrt{2} \sum_{i \in \Lambda} h_{i} Y_{i}, \\
\left\langle X_{A} Y_{B}\right\rangle^{(2)}=\frac{\sum_{\omega_{1} \in \Omega_{\Lambda}} \sum_{\omega_{2} \in \Omega_{\Lambda}} X_{A}\left(\omega_{1}, \omega_{2}\right) Y_{B}\left(\omega_{1}, \omega_{2}\right) e^{-\beta(H(\sigma, \mu))\left(\omega_{1}, \omega_{2}\right)}}{\sum_{\omega_{1} \in \Omega_{\Lambda}} \sum_{\omega_{2} \in \Omega_{\Lambda}} e^{-\beta(H(\sigma, \mu))\left(\omega_{1}, \omega_{2}\right)}} .
\end{gathered}
$$

O denominador da esperança é positivo, logo expandimos a exponencial do numerador da esperança com sua representação em séries de Taylor. Seguimos fatorando cada termo com respeito a $i \in \Lambda$, assim só temos que garantir que

$$
\begin{gathered}
\sum_{\omega_{1} \in \Omega_{\Lambda}} \sum_{\omega_{2} \in \Omega_{\Lambda}} X_{i}^{m}\left(\omega_{1}, \omega_{2}\right) Y_{i}^{n}\left(\omega_{1}, \omega_{2}\right) \\
=\sum_{\omega_{1} \in \Omega_{\Lambda}} \sum_{\omega_{2} \in \Omega_{\Lambda}}\left(\frac{\sigma_{i}\left(\omega_{1}\right)-\mu_{i}\left(\omega_{2}\right)}{\sqrt{2}}\right)^{m}\left(\frac{\sigma_{i}\left(\omega_{1}\right)+\mu_{i}\left(\omega_{2}\right)}{\sqrt{2}}\right)^{n} \geq 0,
\end{gathered}
$$

para quaisquer inteiros $m, n \geq 0$. Se $m$ e $n$ são positivos, então a soma é nula, devido aos valores que assumem os spins $\sigma$ e $\mu$. Se $m$ ou $n$ é zero, então teremos somas em apenas uma destas variáveis $X_{i}$ ou $Y_{i}$, se o ex- 
ponente dessa variável é par então temos que a soma é positiva, e se o exponente é impar, então a soma é nula. Com isso terminamos a prova.

\subsection{Prova da Desigualdade de Simon-Lieb}

Daremos uma prova para uma versão mais fraca do que enunciamos acima. $\mathrm{O}$ argumento que apresentaremos a seguir pode ser encontrado tanto no artigo original de Barry Simon [20] ou com mais detalhes no trabalho de revisão [19]. Para a versão forte desta desigualdade veja a prova dada por Elliot Lieb em [18] ou também as referências [17] e [11].

Teorema 2.2.1. Dado o modelo de Ising d-dimensional com interação de primeiros vizinhos e com Hamiltoniano dado por (1.1), sem campo magnéticos externo(i.e. $h_{t}=0, \forall t \in \Lambda$ ) e com condições de contorno livres. Sejam $i$ e $j$ sítios quaisquer do conjunto finito $\Lambda \subset \mathbb{Z}^{d}$ e seja $\Gamma$ um subconjunto de $\Lambda$, que separa $i$ e $j$. Então

$$
\left\langle\sigma_{i} \sigma_{j}\right\rangle_{\Lambda} \leq \sum_{g \in \Gamma}\left\langle\sigma_{i} \sigma_{g}\right\rangle_{\Lambda}\left\langle\sigma_{g} \sigma_{j}\right\rangle_{\Lambda}
$$

Para demostrar este teorema usaremos um método chamado de "método gráfico", para isso será preciso introduzir novas definições, notações e apresentar alguns lemas.

Seja $\Lambda=\left\{i_{1}, \ldots, i_{k}, \ldots, i_{n}\right\}$ um conjunto finito de sítios. Podemos generalizar o modelo de Ising introduzido no Capítulo anterior de forma que a interação de dois corpos do Hamiltoniano não seja restrita apenas a pares de primeiros vizinhos. Se $E \subset \mathcal{P}(\Lambda)$ denota o conjunto dos pares permitidos, então $E$ determina todas as interações no modelo. Assim podemos pensar no modelo de Ising como sendo definido no grafo simples $(\Lambda=V(G), E=E(G))$.

Analogamente definimos conjunto separador.

- Seja $G$ o grafo simples $(V, E)$. Um subconjunto $\Gamma$ de $\Lambda$ separa o par $i, j \in \Lambda$ se todo caminho em $G$ de $i$ para $j$ possui um sítio em $\Gamma$ e $\{i, j\} \not \subset \Gamma$. 
Ao invés de considerarmos multigrafos, vamos trabalhar com grafos simples "coloridos".

- Uma função $\mathcal{C}: E \rightarrow \mathbb{N} \cup\{0\}$ é chamada de uma coloração das arestas $\operatorname{de} G=(V, E)$.

Se $K$ é um multigrafo, usamos a notação $K \cong(G, \mathcal{C})$ para indicar que $G$ é o grafo simples associado a $K$, através da coloração $\mathcal{C}$.

- Seja $(G, \mathcal{C})$ um grafo colorido. A fronteira de $(G, \mathcal{C})$ é o conjunto $\partial G$ de vértices $i$ de $G$ tal que grau de $i$, definido como $\operatorname{Deg}(i):=\sum_{i \in l} \mathcal{C}(l)$, é um inteiro ímpar.

- Seja $G=(V, E)$ um grafo simples e suponha que $f$ é uma função analítica em $|E|$ variáveis $J_{1}, \ldots, J_{|E|}$. Dada uma coloração $\mathcal{C}$ das arestas de $G$, a derivada de $f$ com respeito a $(G, \mathcal{C})$ é definida por

$$
\partial^{(G, \mathcal{C})} f:=\left.\frac{\partial^{\sum_{l} \mathcal{C}(l)}}{\prod_{l} \partial J_{l}^{\mathcal{C}(l)}} f\right|_{J_{1}=\ldots=J_{|E|}=0}
$$

Simplificaremos um pouco a notação quando for claro qual é o grafo $G$ ou coloração $\mathcal{C}$ que esteja sendo usada. Assim em alguns pontos da prova vamos alternar entre as seguintes notações $\partial^{G} f, \partial^{(G, \mathcal{C})} f$ ou $\partial^{\mathcal{C}} f$.

Será conveniente também estender a noção de coloração para subgrafos.

- Seja $(G, \mathcal{C})$ um grafo colorido. Um grafo colorido $(H, \mathcal{D})$ (com grafo simples associado $H=(V(H), E(H)))$ é um subgrafo colorido de $(G, \mathcal{C})$ se $H$ é um subgrafo de $G$ (no sentido usual) e a restrição de $\mathcal{C}$ a $H$ domina $\mathcal{D}$, isso é,

$$
\mathcal{D}(l) \leq \mathcal{C}(l), \forall l \in E(H) .
$$

O símbolo $\sum_{G \downarrow H}$ será usado para denotar a soma sobre todos os subgrafos coloridos $(H, \mathcal{D})$ de $(G, \mathcal{C})$. Note que o número de subgrafos coloridos $(H, \mathcal{D}) \operatorname{com} \mathcal{D}(H)=\left\{m_{1}, \ldots, m_{|E(G)|}\right\}$ é dado por $\prod_{l \in E(G)}\left(\begin{array}{c}\mathcal{C}(l) \\ m_{l}\end{array}\right)$. Usando isto e a regra de Leibnitz, temos

$$
\partial^{\mathcal{C}}(f g)=\sum_{G \downarrow H}\left(\partial^{H} f\right)\left(\partial^{G \backslash H} g\right),
$$


Onde $f$ e $g$ são funções analíticas em $|E(G)|$ variáveis e $G \backslash H$ é o grafo simples $(V(G), E(G) \backslash E(H))$ com coloração $\left.(\mathcal{C}-\mathcal{D})\right|_{E(G) \backslash E(H)}$.

Para simplificar a notação no restante desta seção vamos escrever simplesmente $\mathcal{H}$ para denotar o Hamiltoniano $H_{\Lambda}$ do modelo de Ising com campo externo nulo. Também usaremos as notações $\langle g\rangle_{0}:=\sum_{\{\sigma\}} g,\langle g\rangle=$ $\frac{\left\langle g e^{-\mathcal{H}}\right\rangle_{0}}{Z}$ e $Z=\left\langle e^{-\mathcal{H}}\right\rangle_{0}$ para denotar os valores esperados (o primeiro não normalizado) com respeito a medida de Gibbs do modelo de Ising generalizado no volume $\Lambda$.

Lema 2.2.1. Seja $\left(H, \mathcal{C}_{H}\right)$ um subgrafo colorido de $G$. Se $d \neq \lambda$, então

$$
\partial^{H}\left\langle\sigma_{d} \sigma_{\lambda} e^{-\mathcal{H}}\right\rangle_{0}=\delta_{\partial H,\{d, \lambda\}} 2^{|\Lambda|},
$$

onde $\delta_{A, B}$ é a função delta de Kronecker.

Demonstração. Por definição de $\partial^{H}$, temos

$$
\left.\frac{\partial^{\sum_{\{a, b\}} \mathcal{C}_{H}(\{a, b\})}}{\prod_{\{a, b\}} \partial J_{\{a, b\}}^{\mathcal{C}_{H}(\{a, b\})}}\left(\left\langle\sigma_{d} \sigma_{\lambda} e^{-\mathcal{H}}\right\rangle_{0}\right)\right|_{J_{k}=0}=\left.\sum_{\sigma} \sigma_{d} \sigma_{\lambda}\left(\prod_{\{a, b\}}\left[\sigma_{a} \sigma_{b}\right]^{\mathcal{C}_{H}(\{a, b\})}\right) e^{-\mathcal{H}}\right|_{J_{k}=0} .
$$

Visto que $\left.e^{-\mathcal{H}}\right|_{J_{1}=\ldots=J_{|E|}=0}=1$ e os outros termos não dependem dos $J_{k}$, temos que o lado direito da igualdade acima é dado por

$$
\sum_{\sigma} \sigma_{d} \sigma_{\lambda} \prod_{\{a, b\}} \sigma_{a}^{\operatorname{Deg}(a)} \sigma_{b}^{\operatorname{Deg}(b)}
$$

Supondo que $\partial H=\{d, \lambda\}$, então podemos afirmar que $d$ e $\lambda$ são vértices de grau ímpar (por definição de $\partial$ ). Desta forma no produtório acima ficamos com os fatores $\sigma_{d}, \sigma_{\lambda}$ e como ainda temos ambos fatores $\sigma_{d} \sigma_{\lambda}$ fora do produtório, segue que

$$
\left.\frac{\partial^{\sum_{\{a, b\}} \mathcal{C}_{H}(\{a, b\})}}{\prod_{\{a, b\}} \partial J_{\{a, b\}}^{\mathcal{C}_{H}(\{a, b\})}}\left(\left\langle\sigma_{d} \sigma_{\lambda} e^{-\mathcal{H}}\right\rangle_{0}\right)\right|_{J_{k}=0}=\sum_{\sigma} 1=2^{|\Lambda|} .
$$

Supondo que $\partial H \neq\{d, \lambda\}$, então temos uma sequência finita de termos $\sigma_{i_{1}}, \sigma_{i_{2}}, \ldots, \sigma_{i_{n}}$ para os quais $i_{k} \neq d, \lambda$ para todo $k$, e cada $\operatorname{Deg}\left(\sigma_{i_{k}}\right)$ é um inteiro ímpar. Se $d$ (ou $\lambda$ ) está em $\partial H$, ficamos com uma potência par de $\sigma_{d}$ (ou $\left.\sigma_{\lambda}\right)$, que não vai alterar o resultado. Portanto podemos afirmar que existem 
inteiros positivos ímpares $o_{1}, \ldots, o_{n}$ tais que

$$
\left.\frac{\partial^{\sum_{\{a, b\}} \mathcal{C}_{H}(\{a, b\})}}{\prod_{\{a, b\}} \partial J_{\{a, b\}}^{\mathcal{C}_{(H}(\{a, b\})}}\left(\left\langle\sigma_{d} \sigma_{\lambda} e^{-\mathcal{H}}\right\rangle_{0}\right)\right|_{J_{k}=0}=\sum_{\sigma} \prod_{k} \sigma_{i_{k}}^{o_{i}}
$$

Também poderíamos ter que $d$ nem $\lambda$ pertencem a $\partial H$, e neste caso o somatório acima teria contribuições com potências ímpares de $\sigma_{d}$ ou $\sigma_{\lambda}$, que faria o efeito apenas de mudar o sinal do somatório acima à direita. Já que os spin só tomam valores 1 ou -1 , é imediato verificar que

$$
\sum_{\sigma} \pm \sigma_{i_{1}}=0
$$

de fato, para toda configuração $\sigma$ em que $\sigma_{i_{1}}=+1$, existe uma $\sigma^{*}$ para a qual $\sigma_{i_{k}}^{*}=-1$. Desta forma cada parcela na soma acima é cancelada. Com isto temos (2.2.1).

Lema 2.2.2. Se $\partial H=\{i, j\}$ então existe um caminho ligando $i$ e $j$.

Demonstração. A fórmula da Soma de Graus afirma: que $\sum_{v \in V(H)} \operatorname{Deg}(v)=$ $2 \sum_{l \in E(H)} \mathcal{C}_{H}(l)$. Essa fórmula implica o Lema do Aperto de Mãos, que fala: o número de vértices de grau ímpar em qualquer grafo colorido $(G, \mathcal{C})$ é par. Vamos usar este fato para provar o lema por contradição. Suponha que não há caminho em $H$ ligando $i$ e $j$, então a componente conexa maximal de $H$ que contém o sítio $i$ não contém o sítio $j$. Olhando para esta componente conexa maximal como um grafo colorido, temos que tal grafo possui um sítio $v$ com $\operatorname{Deg}(v)$ ímpar (que é o sítio $i$ ). Mas isto dá uma contradição com a consequência citada acima do Lema do Aperto de Mãos. Logo existe um caminho $P$ em $H$, ligando $i$ a $j$.

Lema 2.2.3. Seja $P_{0}$ um subgrafo de $H$ fixado. Considere a aplicação $\zeta:\{$ subgrafos de $H\} \rightarrow\{$ subgrafos de $H\}$ definida por $M \mapsto M \Delta P_{0}$. Então $\zeta$ é um automorfismo ${ }^{1}$ de subgrafos de $H$.

Demonstração. Para provar que $\zeta$ é um automorfismo precisamos mostrar que esta aplicação é uma bijeção. Injetividade. Se $M \neq N$, logo há (sem perda de generalidade) $x$ tal que $x \in M$ e $x \notin N$. Se $x \notin P_{0}$, logo $x \in$ $\left(M \cup P_{0}\right) \backslash\left(M \cap P_{0}\right)$ e $x \notin\left(N \cup P_{0}\right) \backslash\left(N \cap P_{0}\right)$. Assim $M \Delta P_{0} \neq N \Delta P_{0}$.

Se $x \in P_{0}$, então $x \notin\left(M \cup P_{0}\right) \backslash\left(M \cap P_{0}\right)$ e $x \in\left(N \cup P_{0}\right) \backslash\left(N \cap P_{0}\right)$. Assim de novo $M \Delta P_{0} \neq N \Delta P_{0}$. Sobrejetividade. Se $N$ é qualquer subgrafo e $P_{0} \subseteq N$, logo $\left(N \backslash P_{0}\right) \Delta P_{0}=\left(\left(N \backslash P_{0}\right) \cup P_{0}\right) \backslash\left(\left(N \backslash P_{0}\right) \cap P_{0}\right)=N \backslash \emptyset=N$, assim $N=\zeta\left(N \backslash P_{0}\right)$.

\footnotetext{
1'é um isomorfismo de grafos coloridos.
} 
Se $P_{0} \nsubseteq N$, logo $\left.\left(N \cup P_{0}\right) \Delta P_{0}=\left(\left(N \cup P_{0}\right) \cup P_{0}\right) \backslash\left(\left(N \cup P_{0}\right) \cap P_{0}\right)=\left(N \cup P_{0}\right) \backslash P_{0}\right)=N$, assim $N=\zeta\left(N \cup P_{0}\right)$.

\section{Demostração da Desigualdade de Simon-Lieb}

Seja $f\left(J_{1}, \ldots, J_{|E(G)|}\right):=\left[\sum_{d \in \Gamma}\left\langle\sigma_{i} \sigma_{d} e^{-\mathcal{H}}\right\rangle_{0}\left\langle\sigma_{d} \sigma_{j} e^{-\mathcal{H}}\right\rangle_{0}\right]-Z\left\langle\sigma_{i} \sigma_{j} e^{-\mathcal{H}}\right\rangle_{0}$. Logo $f$ é analítica, dado que é a combinação linear de exponenciais. Assim é dado pela sua série de MacLaurin. Sabemos que $J_{i} \geq 0$, portanto $f$ toma valores não negativos sempre que suas derivadas de todas as ordens avaliadas em $J_{1}=\ldots=J_{|E(G)|}=0$ sejam não negativas. Se esta condição é cumprida então $\left[\sum_{d \in \beta}\left\langle\sigma_{i} \sigma_{d} e^{-\mathcal{H}}\right\rangle_{0}\left\langle\sigma_{d} \sigma_{j} e^{-\mathcal{H}}\right\rangle_{0}\right] \geq Z\left\langle\sigma_{i} \sigma_{j} e^{-\mathcal{H}}\right\rangle_{0}$ e o teorema segue da divisão por $Z^{2}$.

Observamos que é suficiente mostrar que $\partial^{H} f \geq 0$ para todos os subgrafos coloridos $H$ de $G$.

- Supondo que $\left(H, \mathcal{C}_{H}\right)$ é um grafo colorido com $\partial H=\{i, j\}$.

Neste caso a fronteira é composta apenas por $i$ e $j$, assim temos por uma aplicação direta do Lema (2.2.2) que existe pelo menos um caminho $P$ ligando estes dois sítios.

Continuando, por hipótese, o conjunto $\Gamma$ separa $i$ e $j$ e assim há um sítio $d_{0} \in \Gamma \cap P$. Seja $P_{0}$ o segmento de $P$ ligando $i$ a $d_{0}$, que pode ser escolhido como o segmento de menor comprimento. Note que segue da equação (2.10) e do Lema (2.2.1) que

$$
\partial^{H}\left(\left\langle\sigma_{i} \sigma_{d} e^{-\mathcal{H}}\right\rangle_{0}\left\langle\sigma_{d} \sigma_{j} e^{-\mathcal{H}}\right\rangle_{0}\right) \geq 0
$$

sempre que $d \notin\{i, j\}$. Em particular isto é verdade para todo $d \in \Gamma$ (pela definição de conjunto separador).

Observe que a prova está encerrada se mostrarmos que

$$
\partial^{H}\left[\left\langle\sigma_{i} \sigma_{d_{0}} e^{-\mathcal{H}}\right\rangle_{0}\left\langle\sigma_{j} \sigma_{d_{0}} e^{-\mathcal{H}}\right\rangle_{0}-Z\left\langle\sigma_{i} \sigma_{j} e^{-\mathcal{H}}\right\rangle_{0}\right]=0
$$

para todo $d_{0} \in \Gamma$. De fato, usando esta identidade e somando (2.13) sobre todo os grafos $\Gamma$ temos

$$
\partial^{H}\left(\sum_{d_{0} \in \Gamma}\left\langle\sigma_{i} \sigma_{d_{0}} e^{-\mathcal{H}}\right\rangle_{0}\left\langle\sigma_{j} \sigma_{d_{0}} e^{-\mathcal{H}}\right\rangle_{0}\right)-\sum_{d_{0} \in \Gamma} \partial^{H}\left(Z\left\langle\sigma_{i} \sigma_{j} e^{-\mathcal{H}}\right\rangle_{0}\right)=0 .
$$

Já que a segunda parte não depende de $d_{0}$, temos

$$
\partial^{H}\left(\sum_{d_{0} \in \Gamma}\left\langle\sigma_{i} \sigma_{d_{0}} e^{-\mathcal{H}}\right\rangle_{0}\left\langle\sigma_{j} \sigma_{d_{0}} e^{-\mathcal{H}}\right\rangle_{0}\right)=|\Gamma| \partial^{H}\left(Z\left\langle\sigma_{i} \sigma_{j} e^{-\mathcal{H}}\right\rangle_{0}\right) \geq \partial^{H}\left(Z\left\langle\sigma_{i} \sigma_{j} e^{-\mathcal{H}}\right\rangle_{0}\right) .
$$


Vamos então provar (2.13). Aplicando a regra de Leibntiz (2.10) podemos reescrever o lado esquerdo de (2.13) como segue

$$
\sum_{H \downarrow M} \partial^{M}\left\langle\sigma_{i} \sigma_{d_{0}} e^{-\mathcal{H}}\right\rangle_{0} \partial^{H \backslash M}\left\langle\sigma_{j} \sigma_{d_{0}} e^{-\mathcal{H}}\right\rangle_{0}-\sum_{H \downarrow N} \partial^{N}\left\langle e^{-\mathcal{H}}\right\rangle_{0} \partial^{H \backslash N}\left\langle\sigma_{i} \sigma_{j} e^{-\mathcal{H}}\right\rangle_{0} .
$$

Já que $d_{0} \notin\{i, j\}$, segue da observação (2.2.1) que

$$
2^{2|\Lambda|}\left[\sum_{H \downarrow M} \delta_{\partial M,\left\{i, d_{0}\right\}} \delta_{\partial(H \backslash M),\left\{j, d_{o}\right\}}-\sum_{H \downarrow N} \delta_{\partial N, \emptyset} \delta_{\partial(H \backslash N),\{i, j\}}\right] .
$$

Aplicando o Lema (2.2.3) podemos reescrever (2.14) como mostrado abaixo

$$
2^{2|\Lambda|} \sum_{H \downarrow M}\left[\delta_{\partial M,\left\{i, d_{0}\right\}} \delta_{\partial(H \backslash M),\left\{j, d_{o}\right\}}-\delta_{\partial\left(M \Delta P_{0}\right), \emptyset} \delta_{\partial\left(H \backslash\left(M \Delta P_{0}\right)\right),\{i, j\}}\right] .
$$

Já que tal equação é valida para $d_{0} \in \Gamma$ concluímos finalmente que $\partial^{H} f \geq 0$ para todos os subgrafos coloridos $H$ de $G=(\Lambda=V(G), E(G))$ com fronteira $\partial H=\{i, j\}$. Vamos agora analisar o caso restante.

- Suponha que $\partial H \neq\{i, j\}$.

Como antes, podemos escrever

$$
\partial^{H} f=2^{2|\Lambda|}\left[\sum_{d \in \Gamma} \sum_{H \downarrow M} \delta_{\partial M,\{i, d\}} \delta_{\partial(H \backslash M),\{j, d\}}-\sum_{H \downarrow N} \delta_{\partial N, \emptyset} \delta_{\partial(H \backslash N),\{i, j\}}\right] .
$$

O grau do sítio $k$ em $H$ é dado pela soma de seus graus numa dada partição $\left(\operatorname{Deg}_{H}(k)=\operatorname{Deg}_{M}(k)+\operatorname{Deg}_{H \backslash M}(k)\right)$. Lembremos que a soma de dois inteiros é ímpar se, e só se, um deles é ímpar e o outro par.

Suponha $\delta_{\partial M,\{i, d\}}=\delta_{\partial(H \backslash M),\{j, d\}}=1$. Neste caso, $d$ tem grau ímpar em $M$ e $H \backslash M$, assim tem grau par em $H$. Os sítios $i$ e $j$ tem graus impares em $M$ e $H \backslash M$, respectivamente. Todos os outros sítios tem grau par em ambos $M$ e $H \backslash M$, assim eles têm grau par em $H$. Dado $\{i, d\}=\partial M, j$ tem grau par em $M$ e, similarmente, $i$ tem grau par em $H \backslash M$. Portanto ambos tem graus ímpares em $H$. Mas isto significa que $\partial H=\{i, j\}$, o que é uma contradição. Assim $\delta_{\partial M,\{i, d\}}$ ou $\delta_{\partial(H \backslash M),\{j, d\}}$ é zero, o que significa que seu produto sempre é zero.

Analogamente, se $\delta_{\partial N, \emptyset}=\delta_{\partial(H \backslash N),\{i, j\}}=1, \log i$ e $j$ tem graus pares em $N$ e ímpares em $H \backslash N$, o que significa graus ímpares em $H$. Se $k$ é diferente de $i$ ou $j$, então ele tem um grau par em ambos $N$ e $H \backslash N$ e assim tem grau par em $H$. Assim novamente temos $\partial H=\{i, j\}$, o que é uma contradição. 
Assim o produto $\delta_{\partial N, \emptyset} \delta_{\partial(H \backslash N),\{i, j\}}$ é sempre zero. Assim $\partial^{H} f=0$ para todo subgrafo colorido $H$ de $G \operatorname{com} \partial H \neq\{i, j\}$.

Como $\partial^{H}=0$ quando $\partial H \neq\{i, j\}$ e $\partial^{H} f \geq 0$ quando $\partial H=\{i, j\}$, nós temos que $\partial^{H} f \geq 0$ para todo subgrafo colorido $H$ de $G$. 


\section{Capítulo 3}

\section{Medidas de Gibbs a Volume Infinito e Transição de Fase}

Neste capítulo, apresentamos a teoria necessária para falar de medidas de Gibbs a volume infinito. A abordagem não será pautada em apresentar a teoria em sua máxima generalidade e sim para os casos que nos interessa, logo as chamadas Relações de Consistência, Especificações, Potenciais, etc são discutidos basicamente para o modelo de Ising. Vamos discutir porque não podemos ter a Medidas de Gibbs a volume infinito(no modelo de Ising, por exemplo), apenas tomando uma sequência de Medidas Gibbs a volume finito de forma que elas correspondam a marginais de alguma medida a volume infinito. Também definiremos a transição de fase que é um conceito muito importante na Mecânica Estatística, já que grande quantidade dos problemas estão relacionados a isto, e aqui mostraremos como se expressa a transição de fase mas via descrição das Medidas de Gibbs associadas. Grande parte deste capítulo é inspirada nas referências [22], [14] e também complementamos com alguns exemplos que podem ser encontrados em [16], [12] e [8].

A menos que seja explicitamente mencionado, de agora em diante, $\Lambda$ será um subconjunto finito de $\mathbb{Z}^{d}$ e vamos convencionar que

$$
\Omega_{\Lambda}=\{-1,+1\}^{\Lambda} \quad \text { e } \quad \Omega=\{-1,+1\}^{\mathbb{Z}^{d}}
$$

Função local: Uma função $f: \Omega \rightarrow \mathbb{R}$ é local se existe um subconjunto finito (diferente do conjunto vazio) $A \subset \mathbb{Z}^{d}$ tal que $f(\omega)=f\left(\omega^{\prime}\right)$ sempre que $\omega=\omega^{\prime}$ em $A$ ( i.e. $\left.\omega\right|_{A}=\left.\omega^{\prime}\right|_{A}$ ). 
Estado: Um estado é um funcional linear positivo e normalizado definido em $C(\Omega)$, isto é, uma transformação linear real $f \mapsto\langle f\rangle$ tal que:

1. $\langle 1\rangle=1$;

2. para toda $f$ local tal que $f \geq 0$ temos $\langle f\rangle \geq 0$;

3. se $f, g$ são locais e $\lambda \in \mathbb{R}$, então $\langle f+\lambda g\rangle=\langle f\rangle+\lambda\langle g\rangle$.

O número $\langle f\rangle$ é chamado de media de $f$ no estado $\langle\cdot\rangle$.

Convergência: Sejam $\Lambda_{n} \uparrow \mathbb{Z}^{d}$ e $\eta_{n}$ uma sequência de condições de contorno. Dizemos que a sequência de distribuições de Gibbs $\langle\cdot\rangle_{\Lambda_{n}, \beta, h}^{\eta_{n}}$ converge para o estado $\langle\cdot\rangle$ se, e somente se,

$$
\lim _{n \rightarrow \infty}\langle f\rangle_{\Lambda_{n}, \beta, h}^{\eta_{n}}=\langle f\rangle,
$$

para qualquer função local $f$. O estado $\langle\cdot\rangle$ é chamado estado de Gibbs.

\subsection{Medidas de probabilidade em $\Omega$}

Vamos denotar por $\mathcal{P}\left(\Omega_{\Lambda}\right)$, a coleção de todos os subconjuntos de $\Omega_{\Lambda}$. O conjunto de todas as medidas de probabilidade no espaços mensurável $\left(\Omega_{\Lambda}, \mathcal{P}\left(\Omega_{\Lambda}\right)\right)$ será denotado por $\mathcal{M}_{1}\left(\Omega_{\Lambda}\right)$.

Quando $\triangle \subset S \subset \mathbb{Z}^{d}$ frequentemente representaremos a configuração $\omega_{S} \in \Omega_{S}$ por uma concatenação $\omega_{S}=\omega_{\Delta} \omega_{S \backslash \triangle}$, onde $\left(\omega_{S}\right)_{i}=\left(\omega_{\triangle}\right)_{i}$ se $i \in \triangle \mathrm{e}$ $\left(\omega_{S}\right)_{i}=\left(\omega_{S \backslash \triangle}\right)_{i}$, se $i \in S \backslash \triangle$.

Conjuntos Cilíndricos. Usaremos uma transformação para expressar a restrição de uma configuração a volume infinito a uma configuração a volume finito. Dado $\Lambda \subset \mathbb{Z}^{d}$ finito definimos a "projeção" de $\Omega$ em $\Omega_{\Lambda}$ por

$$
\begin{aligned}
\Pi_{\Lambda}(\omega): \Omega & \rightarrow \Omega_{\Lambda} \\
& \omega \mapsto \Pi_{\Lambda}(\omega):=\left.\omega\right|_{\Lambda}
\end{aligned}
$$

Usando estas projeções podemos definir para cada $\Lambda$ finito, a coleção

$$
\mathcal{C}(\Lambda):=\left\{\Pi_{\Lambda}^{-1}(A): A \in \mathcal{P}\left(\Omega_{\Lambda}\right)\right\}
$$

de todos os eventos que dependem apenas dos spins em $\Lambda$. Cada $C \in \mathcal{C}(\Lambda)$ é um cilindro e o conjunto $\Lambda$ se chamará base do cilindro. 
Observação 3.1.1. $\mathcal{C}(\Lambda)$ é uma álgebra de conjuntos.

Evento local. Um subconjunto de $\Omega$ é chamado de evento local se a ocorrência dele depende apenas de um número finito de variáveis de spins. Exemplo $\mathcal{C}(\Lambda)$.

Definimos também a seguinte coleção

$$
\mathcal{C}_{S}:=\bigcup_{\Lambda \subset S} \mathcal{C}(\Lambda)
$$

onde $S \subset \mathbb{Z}^{d}$ é um subconjunto qualquer e $\Lambda$ é finito.

Observação 3.1.2. Para todo $S, \mathcal{C}_{S}$ tem uma quantidade enumerável de elementos, e tem a estrutura de uma álgebra.

Demonstração. Sabemos que $\mathbb{Z}^{d}$ é um conjunto enumerável. Logo, $S \subset \mathbb{Z}^{d}$ também é enumerável, além disso $\mathcal{C}(\Lambda)$ tem $2^{2^{\Lambda}}$ elementos. Se $S$ é um conjunto infinito, então tem uma quantidade infinita de conjuntos finitos $\left\{\Lambda_{\alpha}\right\}_{\alpha \in I}$. Falta verificar se é uma quantidade infinita enumerável ou não enumerável. Suponhamos que é uma quantidade infinita não enumerável, ou seja, $I$ é um conjunto de índices não enumerável. Assim então tomamos o conjunto $\left\{a_{\alpha}\right\}$ com cada $a_{\alpha} \in \Lambda_{\alpha}$, e com isto teríamos um conjunto não enumerável dentro de $\mathrm{S}$ que é enumerável, o que é uma contradição. Segue que há uma quantidade infinita enumerável de conjuntos finitos. Como a união enumerável de conjuntos finitos $\mathcal{C}(\Lambda)$ é enumerável temos que $\mathcal{C}_{S}$ é um conjunto enumerável.

Para mostrar que $\mathcal{C}_{S}$ é álgebra basta notar que

$\triangleright \emptyset \in \mathcal{C}_{S}$,

$\triangleright$ se $A \in \mathcal{C}_{S}=\bigcup_{\Lambda \subset S} \mathcal{C}(\Lambda)$, temos que $A \in \mathcal{C}(\Lambda)$ para algum $\Lambda \subset S$.

Como $\mathcal{C}(\Lambda)$ é álgebra, temos que $A^{c} \in \mathcal{C}(\Lambda)$, logo $A^{c} \in \mathcal{C}_{S}$.

$\triangleright$ Sejam $\Lambda_{1}, \Lambda_{2}$ subconjuntos finitos de $\mathbb{Z}^{d}$, observamos que

$$
\begin{aligned}
& \Lambda_{1}, \Lambda_{2} \subset \Lambda_{1} \cup \Lambda_{2} \\
& \mathcal{C}_{\Lambda_{1}}, \mathcal{C}_{\Lambda_{2}} \subset \mathcal{C}_{\Lambda_{1} \cup \Lambda_{2}}
\end{aligned}
$$

Se $\Lambda_{1}, \Lambda_{2} \subset S$ temos $\mathcal{C}_{\Lambda_{1}}, \mathcal{C}_{\Lambda_{2}} \subset \mathcal{C}_{S}$ o que implica $\mathcal{C}_{\Lambda_{1} \cup \Lambda_{2}} \subset \mathcal{C}_{S}$

Sejam $A, B \in \mathcal{C}_{S}$, tais que $A \in \mathcal{C}_{\Lambda_{1}}$ e $B \in \mathcal{C}_{\Lambda_{2}}$, isto implica que $A, B \in$ $\mathcal{C}_{\Lambda_{1} \cup \Lambda_{2}}$. Como $\mathcal{C}_{\Lambda_{1} \cup \Lambda_{2}}$ é álgebra, temos que $A \cup B \in \mathcal{C}_{\Lambda_{1} \cup \Lambda_{2}}$, logo $A \cup B \in \mathcal{C}_{S}$. 
Com a coleção $\mathcal{C}_{S}$ geraremos uma $\sigma$-álgebra que denotaremos por

$$
\mathcal{F}_{S}:=\sigma\left(\mathcal{C}_{S}\right)
$$

Quando $S=\mathbb{Z}^{d}$, escrevemos

$$
\begin{aligned}
\mathcal{C} & :=\mathcal{C}_{\mathbb{Z}^{d}} \\
\mathcal{F} & :=\sigma(\mathcal{C}) .
\end{aligned}
$$

O conjunto de todas as medidas de probabilidade definidas no espaço mensurável $(\Omega, \mathcal{F})$ será denotado por $\mathcal{M}_{1}(\Omega, \mathcal{F})$, ou simplesmente por $\mathcal{M}_{1}(\Omega)$.

Distribuição marginal de $\mu$ em $\Lambda$. Dado $\mu \in \mathcal{M}_{1}(\Omega)$ e $\Lambda \subset \mathbb{Z}^{d}$, a Distribuição marginal de $\mu$ em $\Lambda$ é a medida de probabilidade $\mu_{\Lambda} \in \mathcal{M}_{1}\left(\Omega_{\Lambda}\right)$ definida por

$$
\mu_{\Lambda}:=\mu \circ \Pi_{\Lambda}^{-1} .
$$

Vamos precisar também da seguinte definição. Para cada $\triangle \subset \Lambda$, seja $\Pi_{\Delta}^{\Lambda}: \mathcal{P}\left(\Omega_{\Lambda}\right) \rightarrow \mathcal{P}\left(\Omega_{\triangle}\right)$ a aplicação definida por

$$
\Pi_{\triangle}^{\Lambda}:=\Pi_{\triangle} \circ \Pi_{\Lambda}^{-1} .
$$

Relação de consistência. Dizemos que $\left\{\mu_{\Lambda}\right\}_{\Lambda \subset \mathbb{Z}^{d}}, \mu_{\Lambda} \in \mathcal{M}_{1}\left(\Omega_{\Lambda}\right)$ é consistente se para todo $\Lambda$,

$$
\mu_{\triangle}=\mu_{\Lambda} \circ\left(\Pi_{\triangle}^{\Lambda}\right)^{-1}, \text { para todo } \triangle \subset \Lambda
$$

Observe que por construção, as distribuições marginais satisfazem, para todo $\Lambda$, a seguinte igualdade

$$
\left.\mu\right|_{\triangle}:=\left.\mu\right|_{\Lambda} \circ \Pi_{\Lambda}^{-1}, \text { para todo } \triangle \subset \Lambda
$$

Desta forma vemos que as distribuições marginais são consistentes. Veremos logo abaixo que o Teorema da Extensão Kolmogorov, garante a recíproca deste fato isto é, dada uma família consistente de medidas definidas em $\mathcal{M}_{1}\left(\Omega_{\Lambda}\right)$, para todo $\Lambda$ finito, podemos construir uma única medida em $\mathcal{M}_{1}(\Omega)$ cuja as marginais coincidem com a família dada. De maneira mais precisa 
Teorema 3.1.1 (Teorema da extensão de Kolmogorov). Seja $\left\{\mu_{\Lambda}\right\}_{\Lambda \subset \mathbb{Z}^{d}} u m a$ família de medidas que satisfazem as relações de consistência com $\mu_{\Lambda} \in$ $\mathcal{M}_{1}\left(\Omega_{\Lambda}\right)$. Então existe uma única $\mu \in \mathcal{M}_{1}(\Omega)$ tal que $\mu \circ \Pi_{\Lambda}^{-1}=\mu_{\Lambda}$ para toda $\Lambda$.

Observação 3.1.3. No modelo de Ising não é imediato a partir do Teorema da Extensão de Kolmogorov a construção de uma medida $\mu \in \mathcal{M}_{1}(\Omega)$ cujas distribuições marginais, em conjuntos finitos, sejam as medidas $\mu_{\Lambda} \in$ $\mathcal{M}_{1}\left(\Omega_{\Lambda}\right)$ dadas por

$$
\mu_{\Lambda}:=\mu_{\Lambda, \beta, h}^{\emptyset}
$$

A razão é que as medida de Gibbs a volume finito para o modelo de Ising, $\left\{\mu_{\Lambda}\right\}_{\Lambda \subset \mathbb{Z}^{d}}$, não satisfazem as relação de consistência (3.1) se $\beta>0$. Obviamente para $\beta=0$ tais relações são satisfeitas.

\subsubsection{O Modelo de Ising e o Formalismo DLR}

De acordo com a observação feita anteriormente, não é possível construir uma medida no espaço $\mathcal{M}_{1}(\Omega)$, no modelo de Ising, se considerarmos apenas suas distribuições marginais em conjuntos $\Lambda$. Em vista disso, consideraremos a ideia apresentada por Dobrushin, Lamford e Ruelle, que consiste em considerar probabilidades condicionais, ao invés de marginais, já que essas por sua vez satisfazem certa condições de consistência.

O seguinte lema nos ajudara a caracterizar estados de Gibbs a volume infinito sem fazer referência a limites

Lema 3.1.1. Para todo $\triangle \subset \Lambda$, e todas as funções limitadas mensuráveis $f: \Omega \rightarrow \mathbb{R}$, temos

$$
\langle f\rangle_{\Lambda, \beta, h}^{\eta}=\left\langle\langle f\rangle_{\triangle, \beta, h}\right\rangle_{\Lambda, \beta, h}^{\eta}
$$

Demonstração. Para não ter uma notação muito pesada não vamos fazer menção as variáveis $\beta$ e $h$. Cada $\omega \in \Omega_{\Lambda}^{\eta}$ é uma configuração com condições de contorno $\eta$, isto pode ser escrito também como $\omega=\omega_{\Lambda} \eta_{\Lambda^{c}}$, portanto

$$
\left\langle\langle f\rangle_{\triangle, \beta, h}\right\rangle_{\Lambda}^{\eta}=\sum_{\omega_{\Lambda} \in \Omega_{\Lambda}}\langle f\rangle_{\triangle}^{\omega_{\Lambda} \eta_{\Lambda}} \frac{e^{-H_{\Lambda}\left(\omega_{\Lambda} \eta_{\Lambda} c\right)}}{Z_{\Lambda}^{\eta}}
$$

onde

$$
\langle f\rangle_{\triangle}^{\omega_{\Lambda} \eta_{\Lambda^{c}}}=\sum_{\omega_{\triangle}^{\prime} \in \Omega_{\triangle}} f\left(\omega_{\triangle}^{\prime} \omega_{\Lambda \backslash \triangle} \eta_{\Lambda^{c}}\right) \frac{e^{-H_{\Delta}\left(\omega_{\triangle}^{\prime} \omega_{\Lambda \backslash \triangle} \eta_{\Lambda^{c}}\right)}}{Z_{\triangle}^{\omega_{\Lambda} \eta_{\Lambda^{c}}}}
$$


Capítulo 3. Medidas de Gibbs a Volume Infinito e Transição de Fase 30 vamos usar o (3.4) em (3.3) assim temos

$$
\begin{aligned}
& \left\langle\langle f\rangle_{\triangle, \beta, h}\right\rangle_{\Lambda}^{\eta}=\sum_{\omega_{\Lambda} \in \Omega_{\Lambda}} \sum_{\omega_{\Delta}^{\prime} \in \Omega_{\Delta}} f\left(\omega_{\triangle}^{\prime} \omega_{\Lambda \backslash \triangle} \eta_{\Lambda^{c}}\right) \frac{e^{-H_{\Delta}\left(\omega_{\triangle}^{\prime} \omega_{\Lambda \backslash \Delta} \eta_{\Lambda^{c}}\right)}}{Z_{\triangle}^{\omega_{\Lambda} \eta_{\Lambda^{c}}}} \frac{e^{-H_{\Lambda}\left(\omega_{\Lambda} \eta_{\Lambda^{c}}\right)}}{Z_{\Lambda}^{\eta}} \\
& =\sum_{\omega_{\Lambda \backslash \Delta} \in \Omega_{\Lambda \backslash \Delta}} \sum_{\omega_{\Delta} \in \Omega_{\Delta}} \sum_{\omega_{\Delta}^{\prime} \in \Omega_{\Delta}} f\left(\omega_{\triangle}^{\prime} \omega_{\Lambda \backslash \triangle} \eta_{\Lambda^{c}}\right) \frac{\left.e^{-H_{\Delta}\left(\omega_{\triangle}^{\prime} \omega_{\Lambda} \backslash \eta_{\Lambda} c\right.}\right)}{Z_{\Delta}^{\omega_{\Lambda} \eta_{\Lambda^{c}}}} \frac{e^{-H_{\Lambda}\left(\omega_{\Lambda} \eta_{\Lambda} c\right)}}{Z_{\Lambda}^{\eta}} \\
& =\sum_{\omega_{\Lambda \backslash \Delta} \in \Omega_{\Lambda \backslash \Delta}} \sum_{\omega_{\Delta} \in \Omega_{\Delta}} \sum_{\omega_{\Delta}^{\prime} \in \Omega_{\Delta}} f\left(\omega_{\triangle}^{\prime} \omega_{\Lambda \backslash \Delta} \eta_{\Lambda^{c}}\right) \frac{e^{-H_{\Delta}\left(\omega_{\Delta}^{\prime} \omega_{\Lambda \backslash \Delta} \eta_{\Lambda^{c}}\right)}}{Z_{\Delta}^{\omega_{\Lambda} \eta_{\Lambda^{c}}}} \frac{e^{-H_{\Lambda}\left(\omega_{\Lambda} \eta_{\Lambda^{c}}\right)}}{Z_{\Lambda}^{\eta}} \\
& =\sum_{\omega_{\Lambda \backslash \Delta} \in \Omega_{\Lambda \backslash \Delta}} \sum_{\omega_{\Delta} \in \Omega_{\Delta}} \sum_{\omega_{\Delta}^{\prime} \in \Omega_{\Delta}} f\left(\omega_{\triangle}^{\prime} \omega_{\Lambda \backslash \Delta} \eta_{\Lambda^{c}}\right) \frac{e^{-H_{\Delta}\left(\omega_{\triangle}^{\prime} \omega_{\Lambda \backslash \Delta} \eta_{\Lambda^{c}}\right)}}{Z_{\triangle}^{\omega_{\Lambda} \eta_{\Lambda^{c}}}} \frac{e^{-H_{\Lambda}\left(\omega_{\Lambda} \eta_{\Lambda^{c}}\right)}}{Z_{\Lambda}^{\eta}} .
\end{aligned}
$$

Agora vamos denotar $\omega_{\Lambda}=\omega_{\triangle} \omega_{\Lambda \backslash \triangle}$ e usar o fato que

$$
H_{\Lambda}\left(\omega_{\triangle} \omega_{\Lambda \backslash \triangle} \eta_{\Lambda^{c}}\right)-H_{\triangle}\left(\omega_{\triangle} \omega_{\Lambda \backslash \triangle} \eta_{\Lambda^{c}}\right)=H_{\Lambda}\left(\omega_{\triangle}^{\prime} \omega_{\Lambda \backslash \triangle} \eta_{\Lambda^{c}}\right)-H_{\triangle}\left(\omega_{\triangle}^{\prime} \omega_{\Lambda \backslash \triangle} \eta_{\Lambda^{c}}\right),
$$

observe que as interações de spins não dependem de $\omega_{\triangle}$ ou $\omega_{\triangle}^{\prime}$, porque são canceladas devido as subtração que temos acima. Logo o somatório triplo acima pode ser reescrito como

$$
\sum_{\omega_{\Lambda \backslash \Delta} \in \Omega_{\Lambda \backslash \Delta}} \sum_{\omega_{\Delta}^{\prime} \in \Omega_{\Delta}} f\left(\omega_{\triangle}^{\prime} \omega_{\Lambda \backslash \Delta} \eta_{\Lambda^{c}}\right) \frac{e^{-H_{\Lambda}\left(\omega_{\triangle}^{\prime} \omega_{\Lambda \backslash \triangle \eta_{\Lambda} c}\right)}}{Z_{\triangle}^{\omega_{\Delta} \eta_{\Lambda^{c}}}} \frac{\sum_{\omega_{\Delta} \in \Omega_{\Delta}} e^{-H_{\Delta}\left(\omega_{\Delta} \omega_{\Lambda \backslash \Delta} \eta_{\Lambda^{c}}\right)}}{Z_{\Lambda}^{\eta}}
$$

fazendo $\omega_{\Lambda}^{\prime}:=\omega_{\triangle}^{\prime} \omega_{\Lambda \backslash \triangle}$ e algumas simplificações álgebricas ficamos com

$$
\sum_{\omega_{\Lambda}^{\prime} \in \Omega_{\Lambda}} f\left(\omega_{\Lambda}^{\prime} \eta_{\Lambda^{c}}\right) \frac{e^{-H_{\Delta}\left(\omega_{\Lambda}^{\prime} \eta_{\Lambda^{c}}\right)}}{Z_{\Lambda}^{\eta}}=\langle f\rangle_{\Lambda}^{\eta}
$$

As medidas de probabilidade que estamos estudando estão em correspondência com o conjunto de todos os estados definidos em $C(\Omega)$, e isso é o conteúdo do seguinte teorema. 
Teorema 3.1.2. Para cada estado $\langle\cdot\rangle$, existe uma única medida de probabilidade $\mu \in \mathcal{M}_{1}(\Omega)$ tal que

$$
\langle g\rangle=\int_{\Omega} g d \mu
$$

par cada função local $g: \Omega \rightarrow \mathbb{R}$.

Este teorema é o Teorema de Riez-Markov para funções locais e com espaço de spins $\Omega=\{-1,1\}^{\mathbb{Z}^{d}}$. Antes de passar para a prova do Teorema 3.1.2 apresentaremos um lema que será usado para demonstrar o Teorema de Riez-Markov em $\Omega=\{-1,1\}^{\mathbb{Z}^{d}}$.

Lema 3.1.2. Seja $C_{n} \in \mathcal{C}$ uma sequência decrescente de cilindros tal que $\bigcap_{n} C_{n}=\emptyset$. Logo $C_{n}=\emptyset$ para todo $n$ suficientemente grande.

Demonstração. Vamos tomar cilindros $C_{n} \in \mathcal{C}$ da seguinte forma $C_{n}=$ $\Pi_{\Lambda(n)}^{-1}\left(A_{n}\right)$, onde $\Lambda(n) \subset \mathbb{Z}^{d}$ e $A_{n} \in \mathcal{P}\left(\Omega_{\Lambda(n)}\right)$. Suponhamos que $C_{n} \neq \emptyset$ para todo $n$. Por hipótese temos que $C_{m} \subset C_{n}$ para todo $m>n$, seja $\omega^{(i)} \in C_{i}$, com $i \in \mathbb{N}$. Em vista disso observamos que,

$$
\Pi_{\Lambda(n)}\left(\omega^{(m)}\right) \in A_{n}, \quad \forall m>n .
$$

Agora utilizaremos o fato de que $\Omega$ é compacto(3.3.1), assim, existe um $\omega^{*}$ e uma subsequencia $\omega^{\left(n_{k}\right)}$ tal que $\omega^{\left(n_{k}\right)} \rightarrow \omega^{*}$. Logo,

$$
\Pi_{\Lambda(n)}\left(\omega^{*}\right) \in A_{n}, \quad \forall n,
$$

portanto $\omega^{*} \in C_{n}$ para todo $n$. Temos com isto uma contradição com a hipótese, já que $\bigcap_{n} C_{n} \neq \emptyset$.

\section{Demostração do Teorema 3.1 .2 (Riesz-Markov).}

Para toda função continua $f$ temos que $-\|f\| \leq f \leq\|f\|$. Segue das propriedades de linearidade e positividade do funcional $L$ que $|L(f)| \leq\|f\|$. Agora fazemos uma observação crucial que está intimamente ligada com as propriedades topológicas do espaço $\Omega$. Para cada $C \in \mathcal{C}$, temos que $1_{\{C\}} \in C(\Omega)$. Para definir a medida $\mu$ nosso primeiro passo é definir $\mu(C):=L\left(1_{\{C\}}\right)$. Observamos que $0 \leq \mu(C) \leq 1$, e que se $C_{1}, C_{2} \in \mathcal{C}$ são disjuntos, segue que $\mu\left(C_{1} \cup C_{2}\right)=\mu\left(C_{1}\right)+\mu\left(C_{2}\right)$ (por propriedade da função indicadora para conjuntos disjuntos e a linearidade de $L$ ). No livro de [6] temos que se $\mu$ é $\sigma$-aditiva é equivalente a mostrar que é contínua no vazio, usaremos isto para mostrar que $\mu$ é $\sigma$-aditiva com o objetivo de estender $\mu$ a uma medida. Seja $C_{n} \in \mathcal{C}$ tal que $\cap_{n} C_{n}=\emptyset, \Omega=\{-1,1\}^{\mathbb{Z}^{d}}$ ( $\Omega$ é compacto), fazendo uso do 
lema (3.1.2) temos que $C_{n}=\emptyset$ para todo $n$ suficientemente grande, com isto temos que $\mu$ é continua no vazio. Já que $\mu$ é uma função de conjuntos $\sigma$-aditiva na álgebra dos cilindros podemos aplicar o Teorema de Carathéodory e estender unicamente $\mu$ a uma medida na $\sigma$-álgebra gerada pelos cilindros. Também é um resultado clássico da Teoria da Medida que existe uma sequência de funções reais mensuráveis $\left\{f_{n}\right\}$ da forma

$$
f_{n}=\sum_{i=1}^{n} a_{i} 1_{\left\{C_{i}\right\}}, \quad C_{i} \in \mathcal{C},
$$

tal que $\left\|f_{n}-f\right\|_{\infty} \rightarrow 0$. Claramente $\mu\left(f_{n}\right)=L\left(f_{n}\right)$ para todo $n$, e daí segue do Teorema da convergência dominada e da primeira estimativa apresentada no inicio da demostração que

$$
|\mu(f)-L(f)| \leq\left|\mu(f)-\mu\left(f_{n}\right)\right|+\left|L\left(f_{n}\right)-L(f)\right| \leq 2\left\|f_{n}-f\right\|_{\infty} \rightarrow 0,
$$

quando $n \rightarrow \infty$. Logo $\mu(f)=L(f)$ para todo $f \in C(\Omega)$.

Cada estado de Gibbs pode ser colocado em correspondência com uma medida $\mu$. Desta forma a medida tem que satisfazer a condição (3.2). Assim tomando $f=1_{A}$, com $A$ algum evento local, temos

$$
\mu(A)=\int \mu_{\triangle, \beta, h}^{\omega}(A) \mu(d \omega) .
$$

Diremos que $\mu \in \mathcal{M}(\Omega)$ é uma medida de Gibbs a volume infinito para as variáveis $(\beta, h)$, se (3.5) é valida para toda $\triangle \subset \mathbb{Z}^{d}$ finito e todo evento local $A$.

Essa forma de caracterizar e de estudar essas medidas de probabilidade para sistemas de Mecânica Estatística infinitos é muitas vezes conhecido como formalismo DLR em homenagem a Dobrushin, Lanford e Ruelle. 


\subsection{Especificações e Medidas}

Nesta seção vamos falar de uma forma mais geral da abordagem DLR. As medidas $\mu_{\Lambda, \beta, h}^{\bar{\omega}}(\cdot)$ depende do Hamiltoniano do modelo e da condição de contorno, mas muitas propriedades como as que listamos abaixo

$\triangleright$ (3.5) para todo $A \in \mathcal{F}$;

$\triangleright \mu_{\Lambda, \beta, h}^{\omega}(A)$ depende apenas dos valores de $\omega_{i}$ para todos os $i$ que estão fora de $\Lambda$

$\triangleright$ a propriedade que $\forall B \in \mathcal{F}_{\Lambda^{c}}, \mu_{\Lambda, \beta, h}^{\omega}(B)=1_{B}(\omega)$

podem ser introduzidas sem fazer nenhuma referência a um Hamiltoniano.

Daremos as seguintes definições com objetivo de generalizar as propriedades acima. Mais adiante vamos falar de especificação que é a generalização da relação de consistência

Núcleos de Probabilidade. Seja $\Lambda \subset \mathbb{Z}^{d}$ finito. Um núcleo de probabilidade de $\mathcal{F}_{\Lambda^{c}}$ para $\mathcal{F}$ é uma função $\pi_{\Lambda}: \mathcal{F} \times \Omega \rightarrow[0,1]$ com as seguintes propriedades:

$\triangleright$ Para cada $\omega \in \Omega, \pi_{\Lambda}(\cdot \mid \omega)$ é uma medida de probabilidade em $(\Omega, \mathcal{F})$.

$\triangleright$ Para cada $A \in \mathcal{F}, \pi_{\Lambda}(A \mid \cdot)$ é $\mathcal{F}_{\Lambda^{c}}$-mensurável.

Se, além disso,

$$
\pi_{\Lambda}(B \mid \omega)=1_{B}(\omega)
$$

para todo $\omega \in \Omega$ e todo $B \in \mathcal{F}_{\Lambda^{c}}, \pi_{\Lambda}$ será chamado de núcleo próprio.

Exemplo. A aplicação $(E, \bar{\omega}) \mapsto \mu_{\Lambda, \beta, h}^{\bar{\omega}}(E)$ é um núcleo de probabilidade de $\mathcal{F}_{\Lambda^{c}}$ para $\mathcal{F}$.

Observação 3.2.1. No que segue vamos assumir que todos os núcleos são próprios.

Apresentaremos alguns exemplos para mostrar as propriedades dos núcleos 
Capítulo 3. Medidas de Gibbs a Volume Infinito e Transição de Fase 34

Exemplo 3.2.1. Se $\pi_{\Lambda}$ é um núcleo de probabilidade, então para todo $A \in \mathcal{F}$ e todo $B \in \mathcal{F}_{\Lambda^{c}}$ temos que

$$
\pi_{\Lambda}(A \cap B \mid \omega)=\pi_{\Lambda}(A \mid \omega) 1_{B}(\omega) .
$$

Demonstração. Por hipótese, $\pi_{\Lambda}(B \mid \omega)=1_{B}(\omega)$. Observe que

$$
\pi_{\Lambda}(A \cap B \mid \omega) \leq \min \left\{\pi_{\Lambda}(A \mid \omega), 1_{B}(\omega)\right\},
$$

este fato é consequência direta das propriedades de uma medida de probabilidade aplicadas para $\pi_{\Lambda}(\cdot \mid \omega)$ e também do seguinte fato

$$
\min \left\{\pi_{\Lambda}(A \mid \omega), 1_{B}(\omega)\right\}=\pi_{\Lambda}(A \mid \omega) 1_{B}(\omega)=\pi_{\Lambda}(A \mid \omega) \pi_{\Lambda}(B \mid \omega) .
$$

Suponha que a desigualdade em (3.7) seja estrita, então

$$
\pi_{\Lambda}(A \cap B \mid \omega)<\min \left\{\pi_{\Lambda}(A \mid \omega), 1_{B}(\omega)\right\}=\pi_{\Lambda}(A \mid \omega) 1_{B}(\omega)
$$

e da mesma forma que obtemos (3.7), podemos obter

$$
\pi_{\Lambda}\left(A \cap B^{c} \mid \omega\right) \leq \min \left\{\pi_{\Lambda}(A \mid \omega), 1_{B^{c}}(\omega)\right\}=\pi_{\Lambda}(A \mid \omega) 1_{B^{c}}(\omega)
$$

Agora, se somamos as dois ultimas desigualdades obtemos

$$
\pi_{\Lambda}(A \mid \omega)<\pi_{\Lambda}(A \mid \omega)
$$

Daí concluímos que $\pi_{\Lambda}(A \cap B \mid \omega)=\pi_{\Lambda}(A \mid \omega) 1_{B}(\omega)$.

O último exemplo pode ser visto no livro de [16] e na dissertação de [12]

Exemplo 3.2.2. Para todo $A \in \mathcal{F}$ e todo $\omega \in \Omega$,

$$
\pi_{\Lambda}(A \mid \omega)=\sum_{\eta_{\Lambda} \in \Omega_{\Lambda}} \pi_{\Lambda}\left(\Pi_{\Lambda}^{-1}\left(\eta_{\Lambda}\right) \mid \omega\right) 1_{A}\left(\eta_{\Lambda} \omega_{\Lambda^{c}}\right)
$$

E com isso temos que, para toda função mensurável limitada $f: \Omega \rightarrow \mathbb{R}$,

$$
\int f(\eta) \pi_{\Lambda}(d \eta \mid \omega)=\sum_{\eta_{\Lambda} \in \Omega_{\Lambda}} \pi_{\Lambda}\left(\Pi_{\Lambda}^{-1}\left(\eta_{\Lambda}\right) \mid \omega\right) f\left(\eta_{\Lambda} \omega_{\Lambda^{c}}\right) .
$$

Demonstração. Note que 
- Para todo evento $A$ temos que $A=\bigcup_{\eta_{\Lambda} \in \Omega_{\Lambda}}\left[\left(\Pi_{\Lambda}^{-1}\left(\eta_{\Lambda}\right) \cap A\right) \cap \Pi_{\Lambda}^{-1}\left(\eta_{\Lambda}\right)\right]$, $\left(\Pi_{\Lambda}^{-1}\left(\eta_{\Lambda}\right) \cap A\right) \in \mathcal{F}_{\Lambda^{c}}$. Se $\left\{\eta_{\Lambda}\right\},\left\{\eta_{\Lambda}^{\prime}\right\}$ são disjuntos então $\left\{\Pi_{\Lambda}^{-1}\left(\eta_{\Lambda}\right)\right\},\left\{\Pi_{\Lambda}^{-1}\left(\eta_{\Lambda}^{\prime}\right)\right\}$ são disjuntos. Tomando a probabilidade $\pi_{\Lambda}(\cdot \mid \omega)$ ficamos com

$$
\pi_{\Lambda}(A \mid \omega)=\sum_{\eta_{\Lambda} \in \Omega_{\Lambda}} \pi_{\Lambda}\left(\Pi_{\Lambda}^{-1}\left(\eta_{\Lambda}\right) \mid \omega\right) 1_{\left\{\Pi_{\Lambda}^{-1}\left(\eta_{\Lambda}\right) \cap A\right\}}(\omega)
$$

Note que $\Pi_{\Lambda}^{-1}\left(\eta_{\Lambda}\right)$ deixa os valores da configuração finita $\eta_{\Lambda}$ fixos e os de fora não, assim, podemos escrever a igualdade anterior da seguinte maneira

$$
\pi_{\Lambda}(A \mid \omega)=\sum_{\eta_{\Lambda} \in \Omega_{\Lambda}} \pi_{\Lambda}\left(\Pi_{\Lambda}^{-1}\left(\eta_{\Lambda}\right) \mid \omega\right) 1_{A}\left(\eta_{\Lambda} \omega_{\Lambda^{c}}\right)
$$

- Vamos dividir a segunda parte da solução em alguns passos.

1. $f=1_{A}$

$$
\int 1_{A}(\eta) \pi_{\Lambda}(d \eta \mid \omega)=\pi_{\Lambda}(A \mid \omega)=\sum_{\eta_{\Lambda} \in \Omega_{\Lambda}} \pi_{\Lambda}\left(\Pi_{\Lambda}^{-1}\left(\eta_{\Lambda}\right) \mid \omega\right) 1_{A}\left(\eta_{\Lambda} \omega_{\Lambda^{c}}\right) .
$$

2. $f=\sum_{j=1}^{n} a_{j} 1_{A_{j}}$

logo

$$
\begin{aligned}
\int f_{n}(\eta) \pi_{\Lambda}(d \eta \mid \omega) & =\sum_{j=1}^{n} a_{j} \pi_{\Lambda}\left(A_{j} \mid \omega\right) \\
& =\sum_{\eta_{\Lambda} \in \Omega_{\Lambda}} \pi_{\Lambda}\left(\Pi_{\Lambda}^{-1}\left(\eta_{\Lambda}\right) \mid \omega\right) f_{n}\left(\eta_{\Lambda} \omega_{\Lambda^{c}}\right) .
\end{aligned}
$$

3. $f$ é uma função mensurável positiva.

Utilizaremos o seguinte resultado: se $f \geq 0$ é mensurável então existe uma sequencia $\left(f_{n}\right)_{n \geq 1}$ crescente de funções simples, tal que $f=\lim _{n} f_{n}$. Desse modo,

$$
\int f(\eta) \pi_{\Lambda}(d \eta \mid \omega)=\int \lim _{n} f_{n}(\eta) \pi_{\Lambda}(d \eta \mid \omega) .
$$

Pelo teorema da convergência monótona

$$
=\lim _{n} \int f_{n}(\eta) \pi_{\Lambda}(d \eta \mid \omega)
$$


Capítulo 3. Medidas de Gibbs a Volume Infinito e Transição de Fase 36

$$
\begin{aligned}
& =\lim _{n} \sum_{\eta_{\Lambda} \in \Omega_{\Lambda}} \pi_{\Lambda}\left(\Pi_{\Lambda}^{-1}\left(\eta_{\Lambda}\right) \mid \omega\right) f_{n}\left(\eta_{\Lambda} \omega_{\Lambda^{c}}\right) \\
& =\sum_{\eta_{\Lambda} \in \Omega_{\Lambda}} \pi_{\Lambda}\left(\Pi_{\Lambda}^{-1}\left(\eta_{\Lambda}\right) \mid \omega\right) f\left(\eta_{\Lambda} \omega_{\Lambda^{c}}\right)
\end{aligned}
$$

4. $f$ é uma função mensurável e limitada.

$f$ pode ser escrito assim $f=f^{+}-f^{-}$, onde $f^{+}=\sup \{f, 0\}$ e $f^{-}=$ $\sup \{-f, 0\}, \mathrm{e}$

$$
\int f(\eta) \pi_{\Lambda}(d \eta \mid \omega)=\int f^{+}(\eta) \pi_{\Lambda}(d \eta \mid \omega)-\int f^{-}(\eta) \pi_{\Lambda}(d \eta \mid \omega)
$$

com isto e com a parte anterior 3. temos que

$$
\int f(\eta) \pi_{\Lambda}(d \eta \mid \omega)=\sum_{\eta_{\Lambda} \in \Omega_{\Lambda}} \pi_{\Lambda}\left(\Pi_{\Lambda}^{-1}\left(\eta_{\Lambda}\right) \mid \omega\right) f\left(\eta_{\Lambda} \omega_{\Lambda^{c}}\right) .
$$

Como se pode ver com (3.8), o núcleo $\pi_{\Lambda}$ é inteiramente definido pelos números $\pi_{\Lambda}\left(\Pi_{\Lambda}^{-1}\left(\eta_{\Lambda}\right) \mid \omega\right)$.

No que segue vamos usar a notação $\pi_{\Lambda} f$ para denotar a seguinte função $\mathcal{F}_{\Lambda^{c}}$-mensurável

$$
\pi_{\Lambda} f(\omega):=\int_{\Omega} f(\eta) \pi_{\Lambda}(d \eta \mid \omega) .
$$

Composição de Núcleos. Dada a relação de consistência (3.2), vamos convenientemente expressar em termos de composição de Núcleos: dados $\pi_{\Lambda}$ e $\pi_{\triangle}$, denotamos

$$
\pi_{\Lambda} \pi_{\triangle}(A \mid \eta):=\int \pi_{\triangle}(A \mid \omega) \pi_{\Lambda}(d \omega \mid \eta) .
$$

Exemplo 3.2.3. $\pi_{\Lambda} \pi_{\triangle}$, com $\triangle \subset \Lambda$, é um núcleo de probabilidade próprio de $\mathcal{F}_{\Lambda^{c}}$ a $\mathcal{F}$.

Demonstração. Vamos mostrar que para $B \in \mathcal{F}_{\Lambda^{c}}$ temos

$$
\pi_{\Lambda} \pi_{\triangle}(B \mid \eta)=1_{B}(\eta) .
$$

De fato,

$$
\pi_{\Lambda} \pi_{\triangle}(B \mid \eta)=\int \pi_{\triangle}(B \mid \omega) \pi_{\Lambda}(d \omega \mid \eta)
$$


$\pi_{\Lambda}(B \mid \omega)$ é $\mathcal{F}_{\Lambda^{c}}$-mensurável, como $\triangle \subset \Lambda$, temos que $\pi_{\triangle}(B \mid \omega)$ é $\mathcal{F}_{\triangle^{c}}$-mensurável. Já que $\pi_{\triangle}(B \mid \omega)$ é próprio, i.e.

$$
\pi_{\triangle}(B \mid \omega)=1_{B}(\omega)
$$

o lado direito de (3.9) é igual a

$$
\pi_{\Lambda} \pi_{\triangle}(B \mid \eta)=\int 1_{B}(\omega) \pi_{\Lambda}(d \omega \mid \eta)=\pi_{\Lambda}(B \mid \eta)=1_{B}(\eta) .
$$

A próxima definição apresenta um objeto matemático que generaliza as relação de consistência (3.2).

Especificação. Uma especificação é uma família $\pi=\left\{\pi_{\Lambda}\right\}_{\Lambda}$ de núcleos de probabilidades próprios que são consistentes no seguinte sentido

$$
\pi_{\Lambda} \pi_{\triangle}=\pi_{\Lambda}, \quad \forall \triangle \subset \Lambda \subset \mathbb{Z}^{d} .
$$

Dado um núcleo de probabilidade $\pi_{\Lambda}$ e uma medida $\mu \in \mathcal{M}_{1}(\Omega)$, definimos uma nova medida de probabilidade $\mu \pi_{\Lambda} \in \mathcal{M}_{1}(\Omega)$ pela seguinte expressão:

$$
\mu \pi_{\Lambda}(A):=\int \pi_{\Lambda}(A \mid \omega) \mu(d \omega), \quad A \in \mathcal{F} .
$$

Esta nova medida tem a seguinte propriedade.

Proposição 3.2.1. Dada uma medida de probabilidade $\mu \in \mathcal{M}_{1}(\Omega)$ e um núcleo de probabilidade $\pi_{\Lambda}$, Para toda função $f: \Omega \rightarrow \mathbb{R}$ limitada e mensurável, temos que

$$
\mu \pi_{\Lambda}(f)=\mu\left(\pi_{\Lambda} f\right) .
$$

Demonstração. Vamos mostrar a igualdade por passos como fizemos no exemplo (3.2.2), assim

1. $f=1_{A}$ :

vamos considerar $\mu \pi_{\Lambda}$ para a função $1_{A}$. Assim,

$$
\mu \pi_{\Lambda}\left(1_{A}\right)=\int \pi_{\Lambda}(A \mid \omega) \mu(d \omega)
$$

enquanto que

$$
\pi_{\Lambda} 1_{A}(\omega)=\int 1_{A} \pi_{\Lambda}(d \eta \mid \omega)=\pi_{\Lambda}(A \mid \omega) .
$$


Capítulo 3. Medidas de Gibbs a Volume Infinito e Transição de Fase 38

Integrando última igualdade acima com respeito a $\mu \square^{1}$ obtemos a seguinte igualdade

$$
\mu\left(\pi_{\Lambda} 1_{A}\right)=\int \pi_{\Lambda}(A \mid \omega) d \mu
$$

$\log 0$

$$
\mu \pi_{\Lambda}\left(1_{A}\right)=\mu\left(\pi_{\Lambda} 1_{A}\right)
$$

2. $f_{n}=\sum_{j=1}^{n} a_{j} 1_{A_{j}}$ :

$$
\begin{gathered}
\mu \pi_{\Lambda}(f):=\sum_{j=1}^{n} a_{j} \mu \pi_{\Lambda}\left(A_{j}\right)=\sum_{j=1}^{n} a_{j} \int \pi_{\Lambda}\left(A_{j} \mid \omega\right) \mu(d \omega) \\
=\int \sum_{j=1}^{n} a_{j} \pi_{\Lambda}\left(A_{j} \mid \omega\right) \mu(d \omega)=\mu\left(\sum_{j=1}^{n} a_{j} \pi_{\Lambda}\left(A_{j} \mid \omega\right)\right)=\mu\left(\sum_{j=1}^{n} a_{j} \int 1_{A_{j}}(\eta) \pi_{\Lambda}(d \eta \mid \omega)\right) \\
=\mu\left(\int f(\eta) \pi_{\Lambda}(d \eta \mid \omega)\right)=\mu\left(\pi_{\Lambda} f\right) .
\end{gathered}
$$

3. $f \geq 0$ mensurável: neste caso primeiro observamos que

$$
\mu \pi_{\Lambda}(f):=\lim _{n} \sum_{j=1}^{n} a_{j} \mu \pi_{\Lambda}\left(A_{j}\right) .
$$

Usando o Teorema da Convergência Monótona e trabalhando de maneira similar a como fizemos no item 2 , obtemos

$$
\begin{aligned}
\mu \pi_{\Lambda}(f) & =\mu\left(\lim _{n} \int \sum_{j=1}^{n} a_{j} 1_{A_{j}}(\eta) \pi_{\Lambda}(d \eta \mid \omega)\right) \\
& =\mu\left(\int \lim _{n} f_{n}(\eta) \pi_{\Lambda}(d \eta \mid \omega)\right) \\
& \left.=\int \pi_{\Lambda} f(\omega) \mu(d \omega)\right) \\
& =\mu\left(\pi_{\Lambda} f\right) .
\end{aligned}
$$

4. $f$ mensurável e limitada: neste caso decompomos a função $f$ em suas partes positivas e negativas $f=f^{+}-f^{-}$e usamos os itens anteriores

\footnotetext{
${ }^{1}$ Neste capítulo a esperança de uma função $f$ com respeito uma medida de probabilidade $\mu$ poderá ser denotada por $\langle f\rangle_{\mu}, \int f d \mu$ e $\mu(f)$.
} 
para concluir que

$$
\begin{aligned}
\mu\left(\pi_{\Lambda} f\right) & =\mu\left(\pi_{\Lambda} f^{+}\right)-\mu\left(\pi_{\Lambda} f^{-}\right) \\
& =\mu\left(\int \lim _{n} f_{n}^{+}(\eta) \pi_{\Lambda}(d \eta \mid \omega)\right)-\mu\left(\int \lim _{n} f_{n}^{-}(\eta) \pi_{\Lambda}(d \eta \mid \omega)\right) \\
& =\mu\left(\int \lim _{n}\left(f_{n}^{+}(\eta)-f_{n}^{-}(\eta)\right) \pi_{\Lambda}(d \eta \mid \omega)\right) \\
& =\mu\left(\int \lim _{n}\left(f_{n}(\eta)\right) \pi_{\Lambda}(d \eta \mid \omega)\right) \\
& :=\mu \pi_{\Lambda} f .
\end{aligned}
$$

Já tendo falado de especificações podemos falar de medidas que são compatíveis com especificações.

Medida compatível com uma especificação. Seja $\pi=\left\{\pi_{\Lambda}\right\}_{\Lambda \subset \mathbb{Z}^{d}}$ uma especificação. Uma medida $\mu \in \mathcal{M}_{1}(\Omega)$ é compatível com (ou especificada por) $\pi$ se

$$
\mu=\mu \pi_{\Lambda}, \forall \Lambda \subset \mathbb{Z}^{d} .
$$

O conjunto de medidas compatíveis $\operatorname{com} \pi$ (se existir) é denotado por $\mathcal{G}(\pi)$.

Observamos que tínhamos inicialmente a relação de consistência (3.2), esta por sua vez foi generalizada por (3.10). Vimos também que outra forma de escrever (3.2) era dada por (3.5). Agora sabemos como generalizar (3.5) usando (3.11).

Observação 3.2.2. Se $\mu \in \mathcal{G}(\pi)$, então, para todo $A \in \mathcal{F}_{\Lambda}, B \in \mathcal{F}_{\Lambda^{c}}$, temos

$$
\int_{B} \pi_{\Lambda}(A \mid \omega) \mu(d \omega)=\int \pi_{\Lambda}(A \cap B) \mu(d \omega) .
$$

Usando o Exemplo 3.2.1 podemos afirmar que o lado direito da igualdade acima é dado por

$$
\mu \pi_{\Lambda}(A \cap B)=\mu(A \cap B) .
$$

Da definição de probabilidade condicional temos que

$$
\mu(A \cap B)=\int_{B} 1_{A}(\omega) \mu(d \omega)=\int_{B} \mu\left(A \mid \mathcal{F}_{\Lambda^{c}}\right)(\omega) \mu(d \omega) .
$$

Desta forma relacionamos o núcleo $\pi_{\Lambda}$ com a medida de probabilidade 
condicional $\mu$ dada a $\sigma$-álgebra $\mathcal{F}_{\Lambda^{c}}$ da seguinte maneira:

$$
\mu\left(A \mid \mathcal{F}_{\Lambda^{c}}\right)(\cdot)=\pi_{\Lambda}(A \mid \cdot), \quad \mu \text { - quase certamente. }
$$

Esta observação tem consequências mais profundas como, por exemplo, $\mu \in \mathcal{G}(\pi)$ se, e somente se, a especificação $\pi$ fornece uma distribuição condicional regular de $\mu$ dado $\mathcal{F}_{\Lambda^{c}}$ para cada $\Lambda \subset \mathbb{Z}^{d}$. Dito de outra maneira Se $\mu \in \mathcal{G}(\pi)$, então

$$
\mu \pi_{\Lambda}(A)=\int \pi_{\Lambda}(A \mid \omega) \mu(d \omega)=\int \mu(A \mid \omega) \mu(d \omega)=\mu(A),
$$

ou seja $\mu \pi_{\Lambda}=\mu$, este conjunto de equações é conhecido como Equaçoes $D L R$. Observamos que qualquer medida satisfazendo as Equações DLR é tal que suas condicionais com respeito $\mathcal{F}_{\Lambda^{c}}$ são dadas pelo núcleo $\pi_{\Lambda}$ como segue

$$
\mu\left(A \mid \mathcal{F}_{\Lambda^{c}}\right)(\cdot)=\pi_{\Lambda}(A \mid \cdot), \quad \mu \text { - quase certamente. }
$$

\subsubsection{Especificações Gibbsianas}

Na seção anterior mostramos algumas propriedades de especificações bem gerais. Assumimos a existência de tais objetos e deduzimos algumas consequências. O objetivo nesta seção é fornecer uma maneira sistemática de construir especificações.

Vamos começar generalizando o Hamiltoniano do modelo de Ising. Para cada $\omega \in \Omega_{\Lambda}^{\eta}$, a ideia é definir

$$
\mathcal{H}_{\Lambda, \Phi}(\omega):=\sum_{\substack{B \subset \mathbb{Z}^{d} \\ B \cap \AA \emptyset \emptyset}} \Phi_{B}(\omega),
$$

onde, para cada $B$ finito, $\Phi_{B}: \Omega \rightarrow \mathbb{R}$ depende só dos spins dentro de $B$. Como a soma que aparece acima pode ter infinitos termos precisamos garantir que ela seja convergente, para que $\mathcal{H}_{\Lambda, \Phi}(\omega)$ esteja bem definido. Sob a seguinte condição sobre a família $\Phi=\left\{\Phi_{B}\right\}_{B \subset \mathbb{Z}^{d}}$ podemos garantir que o Hamiltoniano acima está bem definido

$$
\sup _{i \in \mathbb{Z}^{d}} \sum_{\substack{B \subset \mathbb{Z}^{d} \\ B \ni i}} \sup _{\omega \in \Omega}\left|\Phi_{B}(\omega)\right|<+\infty .
$$

A família $\Phi=\left\{\Phi_{B}\right\}_{B \subset \mathbb{Z}^{d}}$ será chamada de potencial e quando ela satisfizer 
a condição acima será chamada de Potencial uniformemente absolutamente somável. Se o potencial $\Phi$ é uniformemente absolutamente somável então podemos garantir que a interação de um spin com o resto do sistema é sempre limitada.

Teorema 3.2.1. A família $\pi^{\Phi}=\left\{\pi_{\Lambda}^{\Phi}\right\}_{\Lambda \subset \mathbb{Z}^{d}}$ definida por

$$
\pi_{\Lambda}^{\Phi}\left(\Pi_{\Lambda}^{-1}\left(\tau_{\Lambda}\right) \mid \eta\right):=\frac{1}{Z_{\Lambda, \Phi}^{\eta}} e^{-\mathcal{H}_{\Lambda, \Phi}\left(\tau_{\Lambda} \eta_{\Lambda} c\right)},
$$

onde $Z_{\Lambda, \Phi}^{\eta}$ é a função partição, dada por

$$
Z_{\Lambda, \Phi}^{\eta}:=\sum_{\tau_{\Lambda} \in \Omega_{\Lambda}} e^{-\mathcal{H}_{\Lambda, \Phi}\left(\tau_{\Lambda} \eta_{\Lambda} c\right)} .
$$

define uma especificação. Esta especificação é determinada pelo potencial $\Phi$ e chamada de Especificação Gibbsiana.

Demonstração. A parte mais trabalhosa desta prova consiste em mostrar que são válidas as relações de consistência para este núcleo. A ideia é repetir os passos da prova do Lema 3.2. Para maiores detalhes e também para a prova em casos mais gerais o leitor pode consultar as referências $[8,12,14,16]$.

Exemplo 3.2.4. O potencial associado ao modelo de Ising em $\mathbb{Z}^{d}$ é dado por

$$
\Phi_{B}(\omega)= \begin{cases}-\beta J_{i j} \sigma_{i} \sigma_{j}, & \text { se } A=\{i, j\} \text { e }\|i-j\|=1 \\ -\beta h_{i} \sigma_{i}, & \text { se } A=\{i, j\} \\ 0 & \text { caso contrario }\end{cases}
$$

Medida de Gibbs a volume infinito. Uma medida de probabilidade $\mu$ definida em $(\Omega, \mathcal{F})$ compatível com uma especificação Gibbsiana $\pi^{\Phi}$ é uma medida de Gibbs a volume infinito associada ao potencial $\Phi$.

As seguintes notações serão equivalentes $\mathcal{G}(\Phi)$ e $\mathcal{G}\left(\pi^{\Phi}\right)$. No caso do modelo de Ising já que o potencial $\Phi$ é parametrizado por poucas variáveis $\beta$, $\mathbf{h} \equiv\left\{h_{i}\right\}_{i \in \mathbb{Z}^{d}} \mathbf{e} \mathbf{J} \equiv\left\{J_{i j}\right\}_{i, j \in \mathbb{Z}^{d}}$ podemos denotar seu conjunto de medidas de Gibbs por $\mathcal{G}(\beta, \mathbf{h}, \mathbf{J})$.

Finalmente estamos prontos para dar uma das definições, que sem dúvidas, é uma das mais importantes definições em Mecânica Estatística, a de transição de fase. Vamos nos referir a ela como transição de fase de 
primeira ordem. O termo primeira ordem está ligado a um importante resultado, válido para o modelo de Ising ferromagnético, que relaciona esta definição a uma descontinuidade na derivada de primeira ordem do funcional pressão.

Transição de fase de primeira ordem. O potencial $\Phi$ exibe uma transição de fase de primeira ordem se $\mathcal{G}(\Phi)$ contém ao menos duas medidas de Gibbs distintas.

\subsection{Existência de Medidas em $\mathcal{G}(\pi)$}

A pergunta natural que surge neste momento é: quais são condições necessárias sobre o potencial $\Phi$ para que exista ao menos uma medida compatível $\operatorname{com} \pi^{\Phi}$ ? Daremos mais algumas definições e apresentaremos novos teoremas com a finalidade de responder esta pergunta.

Para responder a essa pergunta vamos precisar estudar um pouco algumas topologias no espaço $\Omega=\{-1,1\}^{\mathbb{Z}^{d}}$, no espaço de funções definidas em $\Omega$ e também no espaço de todas as medidas de probabilidade definidas sobre $\Omega$. Vamos começar introduzindo o seguinte conceito de convergência em $\Omega$.

Convergência em $\Omega$. Seja $\omega \in \Omega$. Uma sequência $\omega^{(n)} \in \Omega$ converge a $\omega$ se, para todo $j \in \mathbb{Z}^{d}$,

$$
\omega_{j}^{(n)} \rightarrow \omega_{j}, \text { quando } n \rightarrow \infty .
$$

Neste caso vamos escrever $\omega^{(n)} \rightarrow \omega$.

Segundo a noção de convergência acima temos que duas configurações estão próximas se elas coincidem em uma região suficientemente grande contendo o origem. A seguir veremos que esta noção de convergência define uma topologia que é metrizável.

Proposição 3.3.1. Para $\omega, \eta \in \Omega$, seja

$$
d(\omega, \eta):=\sum_{i \in \mathbb{Z}^{d}} 2^{-\|i\|_{1}} 1_{\left\{\omega_{i} \neq \eta_{i}\right\}} .
$$

Temos que $d(\cdot, \cdot)$ é uma distancia em $\Omega$, e que $\omega^{(n)} \rightarrow \omega^{*}$ se, e somente se, $d\left(\omega^{(n)}, \omega^{*}\right) \rightarrow 0$.

Demonstração. A função $d$ é um somatório de termos não-negativos então, 
$d \geq 0 . \quad d(\omega, \eta)=0 \Leftrightarrow \omega_{i}=\eta_{i}, \forall i \in \mathbb{Z}^{d} \Leftrightarrow \omega=\eta$. Podemos observar que $d(\omega, \eta)=d(\eta, \omega) .1_{\left\{\omega_{i} \neq \xi_{i}\right\}} \leq 1_{\left\{\omega_{i} \neq \eta_{i}\right\}}+1_{\left\{\eta_{i} \neq \xi_{i}\right\}}$, onde $\omega, \eta, \xi$ são configurações, disso temos $d(\omega, \xi) \leq d(\omega, \eta)+d(\eta, \xi)$. Assim, $d$ é uma distância.

Para a outra afirmação, temos $\omega^{(n)} \rightarrow \omega^{*} \Leftrightarrow \forall \epsilon>0, \exists N_{0} \in \mathbb{N}$ tal que $\mid \omega_{j}^{(n)}-$ $\omega_{j}^{(*)} \mid \leq \epsilon, \forall n \geq N_{0}, \forall j \Leftrightarrow \omega_{j}^{(n)}=\omega_{j}^{*}, \forall n \leq N_{0}, \forall j \Leftrightarrow \sum_{i \in \mathbb{Z}^{d}} 2^{-\|i\|_{1}} 1_{\left\{\omega_{i}^{(n)} \neq \omega_{i}^{*}\right\}} \leq$ $\epsilon, \forall n \geq N_{0} \Leftrightarrow d\left(\omega^{(n)}, \omega^{*}\right) \rightarrow 0$

Em seguida vamos mostrar como usar a compacidade do espaço de spins $\{-1,1\}$, para mostrar que $\Omega$ é compacto com a topologia induzida pela métrica definida acima.

Teorema 3.3.1 (Compacidade de $\Omega$ ). $\Omega$ é sequencialmente compacto: para cada sequência $\omega^{(n)} \in \Omega$, existe $\omega_{*} \in \Omega$ e uma subsequência $\omega^{\left(n_{k}\right)} \rightarrow \omega_{*}$ quando $k \rightarrow \infty$.

Demonstração. Sejam $\omega^{(n)} \in \Omega$ uma configuração e $i_{1}, i_{2}, i_{3}, \cdots$ uma enumeração arbitrária de $\mathbb{Z}^{d}$. A sequência $\left(\omega_{i_{1}}^{(n)}\right)_{n \geq 1}$ é uma sequência em $\{-1,+1\}$, e como este é um conjunto compacto, podemos extrair uma subsequencia $\left(\omega_{i_{1}}^{\left(n_{1}, j\right)}\right)_{j \geq 1}$ a qual converge. Agora consideramos $\left(\omega_{i_{2}}^{\left(n_{1}, j\right)}\right)_{j \geq 1}$, e argumentando como acima podemos garantir a existência de uma subsequência convergente $\left(\omega_{i_{2}}^{\left(n_{2}, j\right)}\right)_{j \geq 1}$, e continuamos assim até ter para cada $k$ uma subsequência convergente $\left(\omega_{i_{k}}^{\left(n_{k}, j\right)}\right)_{j \geq 1}$. Considere a seguinte configuração $\omega^{*} \in \Omega$, definida por

$$
\omega_{i_{k}}^{*}:=\lim _{j \rightarrow \infty} \omega_{i_{k}}^{\left(n_{k, j}\right)},
$$

ou seja, $\omega^{*}$ é uma sequência formada por os limites de cada subsequência $\left(\omega_{i_{k}}^{\left(n_{k}, j\right)}\right)_{j \geq 1}$.

Observe que $\left(\omega^{\left(n_{j}, j\right)}\right)_{j \geq 1}$ é uma subsequência de $\left(\omega^{(n)}\right)_{n \geq 1}$ e além do mais $d\left(\omega^{\left(n_{j}, j\right)}, \omega^{*}\right) \rightarrow 0$, já que $\omega_{k}^{\left(n_{j}, j\right)}=\omega_{k}^{*}$, para todo $k$, o teorema está demostrado.

Função Contínua em $\Omega$. Uma função $f: \Omega \rightarrow \mathbb{R}$ é contínua se para toda sequência $\omega^{(n)} \rightarrow \omega$ temos que $f\left(\omega^{(n)}\right) \rightarrow f(\omega)$. O conjunto de todas as funções contínuas em $\Omega$ será denotado por $C(\Omega)$.

Observação 3.3.1. Em conjuntos compactos uma funções contínuas são também uniformemente contínuas, ou seja, para todo $\epsilon>0$, existe $\delta>0$ tal que $d(\omega, \eta) \leq \delta$ implica que $|f(\omega)-f(\eta)| \leq \epsilon$.

Agora, relacionaremos as funções locais com as funções contínuas. 
Lema 3.3.1. $f \in C(\Omega)$ se, e somete se, existe uma sequência de funções locais $g_{n}$ tal que $2^{2}\left\|g_{n}-f\right\|_{\infty} \rightarrow 0$.

Demonstração. Por hipótese $f: \Omega \rightarrow \mathbb{R}$ é contínua. Fixemos $\epsilon>0$. $f$ é também uniformemente contínua, logo temos que se $d(\omega, \eta)<\delta$, então $|f(\omega)-f(\eta)| \leq \epsilon$. Desta desigualdade temos que existe um $\Lambda$ (finito) tal que se $\eta, \omega$ coincidem em $\Lambda$ então $|f(\omega)-f(\eta)| \leq \epsilon$. Portanto, se fixamos $\hat{\omega} \in \Omega$ e definimos uma função $g$ por $g(\omega):=f\left(\omega_{\Lambda} \hat{\omega}_{\Lambda^{c}}\right)$ temos que $|f(\omega)-g(\omega)| \leq$ $\epsilon, \forall \omega \in \Omega$. e claramente $g$ é uma função local.

Reciprocamente. Como podemos observar toda função local é também contínua, e assim temos que a função local em $\Omega$ é uniformemente contínua. Assim para qualquer $n \in \mathbb{N}$ temos, da hipótese de $g_{n}$ ser uma função local, que para todo $\delta>0$ se $d(\omega, \eta) \leq \delta$ então $\left|g_{n}(\omega)-g_{n}(\eta)\right| \leq \epsilon$. Por hipótese, temos que $g_{n}$ satisfaz que $\left\|g_{n}-f\right\| \rightarrow 0$, daí qualquer $\epsilon>0$ fixado podemos garantir que existe $n$ tal que $\left\|g_{n}-f\right\|_{\infty} \leq \epsilon$. Já que para todo par $\omega, \eta$ satisfazendo $d(\omega, \eta) \leq \delta$ temos que

$$
|f(\omega)-f(\eta)| \leq\left|f(\omega)-g_{n}(\omega)\right|+\left|g_{n}(\omega)-g_{n}(\eta)\right|+\left|f(\eta)-g_{n}(\eta)\right| \leq 3 \epsilon,
$$

podemos concluir que $f$ é contínua.

O próximo lema mostra propriedades interessantes das medidas de probabilidade em $(\Omega, \mathcal{F})$, que são unicamente determinadas por sua ação em conjuntos cilíndricos ou em funções contínuas.

Lema 3.3.2. Se $\mu, \nu \in \mathcal{M}_{1}(\Omega)$, então os seguintes itens são equivalentes:
a) $\mu=\nu$
b) $\mu(C)=\nu(C)$ para todo $C \in \mathcal{C}$.
c) $\mu(g)=\nu(g)$ para toda função local $g$
d) $\mu(f)=\nu(f)$ para toda $f \in C(\Omega)$.

Demonstração. Primeiro $a) \Rightarrow b$ ) é óbvio. A recíproca, $b) \Rightarrow a$ ) é consequência da unicidade do Teorema de Carathéodory que garante que se $\mathcal{F}$ é gerada por uma álgebra $\mathcal{C}$, ou seja, $\mathcal{F}:=\sigma(\mathcal{C})$, e $\mu, \nu$ são medidas de probabilidade em $(\Omega, \mathcal{F})$ que coincidem em $\mathcal{C}$, então as duas medidas coincidem em $\mathcal{F}$. Para ver que $b) \Leftrightarrow c$ ) basta notar que a função $1_{C}$, ou seja, a indicadora do cilindro $C$, é uma função local. A implicação $c) \Rightarrow d$ ) segue do

$$
{ }^{2}\|f\|_{\infty}:=\sup _{\omega \in \Omega}|f(\omega)|
$$


lema anterior. De fato, dada uma função contínua $f$ sabemos que existe sequência de funções locais $g_{n}$ tais que $\left\|g_{n}-f\right\|_{\infty} \rightarrow 0$. Assim temos para qualquer $\epsilon>0$ fixado que se $n$ é suficientemente grande que $\left|g_{n}\right| \leq \epsilon+|f|$. Usando agora que $\mu\left(g_{n}\right)=\nu\left(g_{n}\right)$ segue do Teorema da Convergência Dominada que $\mu(f)=\nu(f)$. A última implicação $d) \Rightarrow c$ ) segue diretamente do fato de toda função local ser uma função contínua.

\subsubsection{Algumas Propriedades de $\mathcal{M}_{1}(\Omega)$}

Convergência em $\mathcal{M}_{1}(\Omega)$. Vamos dizer que uma sequência $\mu_{n} \in \mathcal{M}_{1}(\Omega)$ converge para $\mu \in \mathcal{M}_{1}(\Omega)$ se $\mu_{n}(C) \rightarrow \mu(C)$, para todos os cilindros $C \in$ $\mathcal{C}$. Neste caso vamos escrever $\mu_{n} \Rightarrow \mu$. Essa convergência é conhecida também como convergência fraca.

Teorema 3.3.2 (Compacidade do espaço $\mathcal{M}_{1}(\Omega)$ ). Com a noção de convergência fraca, $\mathcal{M}_{1}(\Omega)$ é sequencialmente compacto, isto é, para cada sequência $\mu_{n} \in \mathcal{M}_{1}(\Omega)$, existe $\mu \in \mathcal{M}_{1}(\Omega)$ e uma subsequencia $\mu_{n_{k}}$ tal que $\mu_{n_{k}} \Rightarrow \mu$ quando $k \rightarrow \infty$.

Demonstração. Sabemos que $\mathcal{C}$ tem uma quantidade enumerável de elementos pela Observação 3.1.2. Seja $C_{1}, C_{2}, \cdots$ uma enumeração arbitrária de todos os cilindros de $\mathcal{C}$. Note que a sequência $\mu_{n}\left(C_{1}\right)$, que toma valores no compacto $[0,1]$. Portanto podemos garantir que existe uma subsequência convergente $\mu_{n_{1}, j}\left(C_{1}\right)$ e denotaremos seu limite por

$$
\mu\left(C_{1}\right):=\lim _{j \rightarrow \infty} \mu_{n_{1}, j}\left(C_{1}\right) .
$$

Agora, no cilindro $C_{2}$, extraímos da sequência $\mu_{n_{1}, j}\left(C_{2}\right) \in[0,1]$ uma subsequência convergente $\mu_{n_{2}, j}\left(C_{2}\right)$ cujo limite será chamado de

$$
\mu\left(C_{2}\right):=\lim _{j \rightarrow \infty} \mu_{n_{2}, j}\left(C_{2}\right) .
$$

Continuamos indutivamente este processo e para o cilindro $C_{k}, k \geq 1$, extraímos uma subsequência convergente $\mu_{n_{k}, j}\left(C_{k}\right)$ cujo o limite será denominado

$$
\mu\left(C_{k}\right):=\lim _{j \rightarrow \infty} \mu_{n_{k}, j}\left(C_{k}\right) .
$$

Se consideramos a sequencia diagonal $n_{j, j}$, temos que $\mu_{n_{j}, j}(C) \rightarrow \mu(C)$ para todo $C \in \mathcal{C}$. A função de conjunto $\mu$ que acabamos de definir está bem definida em toda álgebra $\mathcal{C}$, assim mostrar que ela admite uma extensão a 
uma medida definida na $\sigma$-álgebra $\mathcal{F}$. Para isso basta procedermos como na prova do Teorema 3.3.1, fazer uso do Lema 3.1.2 para verificar que $\mu(\cdot)$ é $\sigma$-aditiva em $\mathcal{C}$ e em seguida, usar o Teorema de Extensão de Carathéodory para estender $\mu$ a uma probabilidade em $\mathcal{F}$. Por construção temos que $\mu_{n_{j}, j} \Rightarrow \mu$.

\subsubsection{Especificação $\pi \operatorname{com} \mathcal{G}(\pi) \neq \emptyset$}

Especificação quasilocal. Uma especificação $\pi=\left\{\pi_{\Lambda}\right\}_{\Lambda \subset \mathbb{Z}^{d}}$ é quasilocal se cada núcleo $\pi_{\Lambda}$ é contínuo com respeito a sua segunda entrada. Isto é, se para todo $C \in \mathcal{C}$, a função $\omega \mapsto \pi_{\Lambda}(C \mid \omega)$ pertence a $C(\Omega)$.

Teorema 3.3.3. Uma especificação $\pi^{\Phi}$ associada a um potencial $\Phi$ absolutamente uniformemente somável é quasilocal. Se $\pi=\left\{\pi_{\Lambda}\right\}_{\Lambda \subset \mathbb{Z}^{d}}$ é quasilocal, para quaisquer $\Lambda \subset \mathbb{Z}^{d}$ finito e $f \in C(\Omega)$ fixados temos que $\pi_{\Lambda} f \in C(\Omega)$.

Demonstração. Para a prova deste fato veja, [12, 14, 16].

Teorema 3.3.4. Se $\pi=\left\{\pi_{\Lambda}\right\}_{\Lambda \subset \mathbb{Z}^{d}}$ é uma especificação quasilocal, então $\mathcal{G}(\pi) \neq \emptyset$.

Demonstração. Seja $\omega \in \Omega$ fixado e considere a seguinte sequência de medidas $\mu_{n}(\cdot):=\pi_{\Lambda_{n}}(\cdot \mid \omega)$. Como os núcleos satisfazem a relação de consistência, temos para todo $n$ tal que $\Lambda \subset \Lambda_{n}$ que

$$
\mu_{n} \pi_{\Lambda}=\pi_{\Lambda_{n}} \pi_{\Lambda}(\cdot \mid \omega)=\pi_{\Lambda_{n}}(\cdot \mid \omega)=\mu_{n} .
$$

Agora, fazendo uso da compacidade de $\mathcal{M}_{1}(\Omega)$ ( Teorema 3.3.2) podemos garantir que para a sequência $\mu_{n} \in \mathcal{M}_{1}(\Omega)$ existe uma medida $\mu \in \mathcal{M}_{1}(\Omega)$ e uma subsequência $\mu_{n_{k}}$ tais que $\mu_{n_{k}} \Rightarrow \mu$ quando $k \rightarrow \infty$. Nosso objetivo é mostrar que $\mu \in \mathcal{G}(\pi)$, i.e. $\mu \pi_{\Lambda}=\mu$ para todo $\Lambda$ finito. Seja $f \in C(\Omega)$. Como $\pi$ é quasilocal segue do Teorema 3.3 .3 que $\pi_{\Lambda} f \in C(\Omega)$. Logo,

$$
\mu \pi_{\Lambda}(f)=\mu\left(\pi_{\Lambda} f\right)=\lim _{k \rightarrow \infty} \mu_{n_{k}}\left(\pi_{\Lambda} f\right)=\lim _{k \rightarrow \infty} \mu_{n_{k}} \pi_{\Lambda}(f)=\lim _{k \rightarrow \infty} \mu_{n_{k}}(f)=\mu(f) .
$$

Pelo Lema 3.3.2 e a igualdade acima temos que $\mu \pi_{\Lambda}=\mu$, para todo $\Lambda$ finito e portanto $\mu \in \mathcal{G}(\pi)$. 


\section{Parte II}

\section{Desigualdades Diferenciais}





\section{Capítulo 4}

\section{Representações por Correntes e Passeios aleatórios}

Neste capítulo veremos as definições e as propriedades de duas formas de representação. Estas formas de representar quantidades físicas nos ajudará quando tivermos que deduzir e estender as desigualdades principais nos próximos dois capítulos. As referências mais usadas aqui são [1], [3], [5], e [22].

\subsection{Representações por Correntes Aleatórias}

Vamos começar o estudos da representações no modelo de Ising, quando sem campo magnético $h=0$. Vamos mostrar como podemos expressar a função de partição em termos de configurações de correntes. Um numero de fluxo é uma função

$$
\begin{aligned}
n .: \quad \mathcal{E}_{\Lambda} & \rightarrow \mathbb{N} \cup\{0\} \\
\{x, y\} & \mapsto n_{\{x, y\}}
\end{aligned}
$$

onde $\mathcal{E}_{\Lambda}$ é o conjunto de arestas. O número natural ou $0, n_{\{x, y\}}$ é também conhecido como simplesmente fluxo na aresta $\{x, y\}$. Uma configuração de correntes será um conjunto de números de fluxo com respeito a certo grafo $\left(\Lambda, \mathcal{E}_{\Lambda}\right)$, e sua notação será $\mathbf{n}=\left(n_{e}\right)_{e \in \mathcal{E}_{\Lambda}}$, onde $e=\{x, y\} \in \mathcal{E}_{\Lambda}$ denota uma aresta genérica do grafo $\left(\Lambda, \mathcal{E}_{\Lambda}\right)$.

Quando o campo magnético não for nulo pode-se expressar as correlações e a função de partição por meio das configurações de correntes fazendo uma pequena modificação no grafo $\left(\Lambda, \mathcal{E}_{\Lambda}\right)$ que consiste basicamente em criar um novo grafo onde adicionamos um sítio $g$ (o símbolo $g$ será reservado para o sítio fantasma) e o ligamos a todos os demais 
vértices. O spin neste sítio se chamará "spin fantasma", e veremos que este novo modelo pode ser pensado como um modelo sem campo, ou seja, $h=0$. O spin fantasma estará conectando a todos os sítios da rede, por arestas da forma $\{x, g\}$ e as correntes correspondentes a estas arestas serão definidas em termos de $J_{\{x, g\}}=h$, além disso as arestas que conectam os sítios da rede ao spin fantasma $(\{x, g\})$ serão chamadas de $h$-arestas. Para fixar notação o grafo obtido pela adição do sítio fantasma e será denotado por $\left(\Lambda^{g}, \overline{\mathcal{E}_{\Lambda}}\right)$, onde $\Lambda^{g}:=\Lambda \cup\{g\}$ e $\overline{\mathcal{E}_{\Lambda}}:=\mathcal{E}_{\Lambda} \cup \mathcal{E}_{\Lambda}^{g}, \operatorname{com} \mathcal{E}_{\Lambda}^{g}=\{\{x, g\}: x \in \Lambda\}$.

Observação 4.1.1. Neste capitulo e os dois seguintes faremos as provas a volume finito, os resultados se podem estender a volume infinito tomando o limite termodinâmico.

De agora em diante vamos voltar nossa atenção para modelos de Ising a primeiros vizinhos (embora o analise também funciona para modelos de longo alcance) ferromagnéticos com constantes de acoplamento invariantes por translação (da forma $J_{x y}=J(x-y)$ ) com condições de fronteira livre definido em $\Lambda \subset \mathbb{Z}^{d}$, pelo seguinte Hamiltoniano

$$
\mathcal{H}_{\Lambda, h}(\omega):=-\frac{1}{2} \sum_{\{x, y\} \in \mathcal{E}_{\Lambda}} J(x-y) \omega_{x} \omega_{y}-h \sum_{x \in \Lambda} \omega_{x}
$$

Ao invés de considerar que a medida a priori é a medida de contagem em $\{-1,1\}$, vamos considerá-la como sendo uma Bernoulli de parâmetro 1/2. Desta forma a função de partição é dada por

$$
Z_{\Lambda}(\beta, h):=2^{-|\Lambda|} \sum_{\omega \in \Omega_{\Lambda}} e^{-\beta \mathcal{H}_{\Lambda, h}(\omega)}
$$

Com objetivo de obter uma notação simples para as representações em correntes aleatórias introduzimos as seguintes notações

$$
w(\mathbf{n}):=\prod_{b \in \overline{\mathcal{E}}_{\Lambda} g} \frac{\left(\beta J_{b}\right)^{n_{b}}}{n_{b} !}, \quad \text { onde } \quad J_{b}= \begin{cases}J(y-x), & \text { se } b=\{x, y\} \\ h, & \text { se } b=\{x, g\} .\end{cases}
$$

Fontes de uma configuração de correntes $\mathbf{n}$. Um elemento $x \in \Lambda^{g}$ tal que $\sum_{b \ni x} n_{b}$ é ímpar, será chamado de uma fonte de $\mathbf{n}$. O conjunto de todas as fontes de $\mathbf{n}$ será denotado por $\partial \mathbf{n}$. 
Lema 4.1.1. Para todo $\beta>0$ e $h \geq 0$, temos que

$$
Z_{\Lambda}(\beta, h)=\sum_{\partial \boldsymbol{n}=\emptyset} w(\boldsymbol{n})
$$

Demonstração. Se $b \in \mathcal{E}_{\Lambda}^{g}$, isto é, uma aresta contendo o vértice fantasma da forma $\{x, b\}$ então vamos usar a notação $J_{b} \omega_{x} \omega_{g}$ para indicar $J_{b} \omega_{x}$. Desta forma podemos escrever

$$
\begin{aligned}
Z_{\Lambda}(b, h) & =\frac{1}{2^{|\Lambda|}} \sum_{\omega \in \Omega_{\Lambda}} \exp \left(\beta \sum_{b=(x, y) \in \overline{\mathcal{E}}_{\Lambda}} J_{b} \omega_{x} \omega_{y}\right) \\
& =\frac{1}{2^{|\Lambda|}} \sum_{\omega \in \Omega_{\Lambda}} \prod_{b=\{x, y\} \in \overline{\mathcal{E}}_{\Lambda}} \exp \left(\beta J_{b} \omega_{x} \omega_{y}\right)
\end{aligned}
$$

Pela Fórmula de Taylor, temos que o lado direito da igualdade acima é dado por

$$
\frac{1}{2^{|\Lambda|}} \sum_{\omega \in \Omega_{\Lambda}} \prod_{b=\{x, y\} \in \overline{\mathcal{E}}_{\Lambda}} \sum_{n=0}^{\infty} \frac{\left(\beta J_{b} \omega_{x} \omega_{y}\right)^{n}}{n !}=\frac{1}{2^{|\Lambda|}} \sum_{\omega \in \Omega_{\Lambda}} \sum_{\mathbf{n}} \prod_{b=\{x, y\} \in \overline{\mathcal{E}}_{\Lambda}} \frac{\left(\beta J_{b}\right)^{n_{b}}\left(\omega_{x} \omega_{y}\right)^{n_{b}}}{n_{b} !}
$$

onde $\mathbf{n}=\left(n_{b}\right)_{b \in \overline{\mathcal{E}}_{\Lambda}}$. Separando o produtório aparecendo no lado direito da igualdade acima ficamos com

$$
Z_{\Lambda}(\beta, h)=\frac{1}{2^{|\Lambda|}} \sum_{\omega \in \Omega_{\Lambda}} \sum_{\mathbf{n}} \prod_{b \in \overline{\mathcal{E}}_{\Lambda}} \frac{\left(\beta J_{b}\right)^{n_{b}}}{n_{b} !} \prod_{b=\{x, y\} \in \overline{\mathcal{E}}_{\Lambda}}\left(\omega_{x} \omega_{y}\right)^{n_{b}},
$$

Pela definição $w(\mathbf{n})$ a expressão acima pode ser reescrita como

$$
\begin{aligned}
\frac{1}{2^{|\Lambda|}} \sum_{\omega \in \Omega_{\Lambda}} \sum_{\mathbf{n}} w(\mathbf{n}) \prod_{\{x, y\} \in \overline{\mathcal{E}}_{\Lambda}}\left(\omega_{x} \omega_{y}\right)^{n_{b}} & =\frac{1}{2^{|\Lambda|}} \sum_{\mathbf{n}} w(\mathbf{n}) \sum_{\omega \in \Omega_{\Lambda}} \prod_{b=\{x, y\} \in \overline{\mathcal{E}}_{\Lambda}}\left(\omega_{x} \omega_{y}\right)^{n_{b}} \\
& =\frac{1}{2^{|\Lambda|}} \sum_{\mathbf{n}} w(\mathbf{n}) \sum_{\omega \in \Omega_{\Lambda}} \prod_{x \in \Lambda}\left(\omega_{x}\right)^{\sum_{b \ni x} n_{b}} .
\end{aligned}
$$

Usando o Teorema de Fubuni podemos reescrever o somatório aparecendo depois de $w(\mathbf{n})$ no lado direito da igualdade acima como segue

$$
\sum_{\omega \in \Omega_{\Lambda}} \prod_{x \in \Lambda}\left(\omega_{x}\right)^{\sum_{\ni \ni x} n_{b}}=\prod_{x \in \Lambda} \sum_{\omega_{x} \in\{-1,1\}}\left(\omega_{x}\right)^{\sum_{b \ni x} n_{b}}=2^{|\Lambda|} 1\left\{\sum_{b \ni x} n_{b} \text { é par } \forall x \in \Lambda\right\} .
$$


Com esta identidade temos finalmente a igualdade desejada

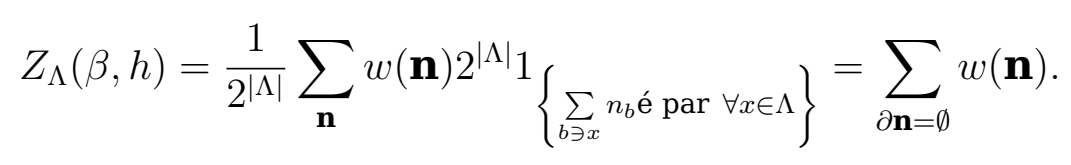

Lema 4.1.2. Para a função de correlação $\left\langle\sigma_{A}\right\rangle_{\Lambda}$, onde $\sigma_{A}=\prod_{x \in A} \sigma_{x}$, temos que:

$$
\left\langle\sigma_{A}\right\rangle_{\Lambda}=\frac{\sum_{\boldsymbol{n}=A} w(\boldsymbol{n})}{\sum_{\partial \boldsymbol{n}=\emptyset} w(\boldsymbol{n})}, \text { se }|A| \text { é par }
$$

$e$

$$
\left\langle\sigma_{A}\right\rangle_{\Lambda}=\frac{\sum_{\partial \boldsymbol{n}=A \cup\{g\}} w(\boldsymbol{n})}{\sum_{\partial \boldsymbol{n}=\emptyset} w(\boldsymbol{n})}, \text { se }|A| \text { é ímpar }
$$

Demonstração. Por definição temos que

$$
\left\langle\sigma_{A}\right\rangle_{\Lambda}=\frac{\sum_{\omega \in \Omega_{\Lambda}} \sigma_{A}(\omega) e^{-\beta \mathcal{H}_{\Lambda, h}(\omega)}}{\sum_{\omega \in \Omega_{\Lambda}} e^{-\beta \mathcal{H}_{\Lambda, h}(\omega)}} .
$$

Claramente só precisamos fazer a expansão em correntes para o numerador pois, a expansão do denominador foi obtida no lema anterior. Vamos trabalhar no numerador de maneira análoga a que fizemos no Lema 4.1.1. Lembrando que $\sigma_{x}(\omega)=\omega_{x}$, o numerador do valor esperado acima pode ser reescrito como

$$
\begin{aligned}
\sum_{\omega \in \Omega_{\Lambda}} \sigma_{A}(\omega) e^{-\beta \mathcal{H}_{\Lambda, h}(\omega)} & =\sum_{\mathbf{n}} w(\mathbf{n}) \sum_{\omega \in \Omega_{\Lambda}} \sigma_{A}(\omega) \prod_{b=\{x, y\} \in \overline{\mathcal{E}}_{\Lambda}}\left(\omega_{x} \omega_{y}\right)^{n_{b}} \\
& =\sum_{\mathbf{n}} w(\mathbf{n}) \sum_{\omega \in \Omega_{\Lambda}} \sigma_{A}(\omega) \prod_{x \in \Lambda}\left(\omega_{x}\right)^{\sum_{b \ni x} n_{b}} \\
& =\sum_{\mathbf{n}} w(\mathbf{n}) \sum_{\omega \in \Omega_{\Lambda}} \prod_{x \in \Lambda}\left(\omega_{x}\right)^{\sum_{b \ni x} n_{b}+1_{A}(x)}
\end{aligned}
$$


Pelo Lema do aperto de mãos (ver (2.2.2)) temos que

$$
\sum_{x \in \Lambda^{g}}\left(\sum_{b \ni x} n_{b}\right) \equiv 0(\bmod 2) \quad \Longrightarrow \quad \sum_{x \in \Lambda}\left(\sum_{b \ni x} n_{b}\right)+\sum_{b \ni g} n_{b} \equiv 0(\bmod 2)
$$

e portanto podemos afirmar que ambas a somas do lado direito acima têm a mesma paridade, isto é,

$$
\sum_{x \in \Lambda}\left(\sum_{b \ni x} n_{b}\right) \equiv \sum_{b \ni g} n_{b} \quad(\bmod 2)
$$

Fixe uma configuração de correntes $\mathbf{n}$. Se $|A|$ é par e todos os expoentes que aparecem na expressão abaixo são pares

$$
\sum_{\omega \in \Omega_{\Lambda}} \prod_{x \in \Lambda}\left(\omega_{x}\right)^{\sum_{b \ni x} n_{b}+1_{A}(x)}
$$

temos: que o somatório acima é não-nulo, que todo $x \in A$ pertence também a $\partial \mathbf{n}$ e além do mais $\sum_{b \ni g} n_{b}$ é par. Assim no caso $|A|$ par, cada configuração de corrente para a qual a soma acima é não nula é tal que $\partial \mathbf{n}=A$. Por outro lado, se $|A|$ é ímpar e o somatório em (4.8) é não nulo, então podemos afirmar que todo $x \in A$ também pertence a $\partial \mathbf{n}$. Além do mais teremos

$$
|A|+\sum_{x \in \Lambda} \sum_{b \ni x} n_{b}=\sum_{x \in \Lambda} \sum_{b \ni x} n_{b}+1_{A}(x) \equiv 0(\bmod 2) .
$$

Já que estamos assumindo que $|A|$ é ímpar segue da congruência (4.7) que $\sum_{b \ni g} n_{b}$ é ímpar e portanto $g \in \partial \mathbf{n}$. Logo concluímos que se $|A|$ é ímpar e a soma (4.8) é não nula então $\partial \mathbf{n}=A \cup\{g\}$. Em resumo temos

$$
\sum_{\mathbf{n}} w(\mathbf{n}) \sum_{\omega \in \Omega_{\Lambda}} \prod_{x \in \Lambda}\left(\omega_{x}\right)^{\sum_{b \ni x} n_{b}+1_{A}(x)}= \begin{cases}2^{|\Lambda|} \sum_{\mathbf{n}} w(\mathbf{n}) 1_{\{\partial \mathbf{n}=A\}}, & \text { se }|A| \text { é par; } \\ 2^{|\Lambda|} \sum_{\mathbf{n}} w(\mathbf{n}) 1_{\{\partial \mathbf{n}=A \cup\{g\}\}}, & \text { se }|A| \text { é ímpar. }\end{cases}
$$

Como já sabemos do lema anterior que vale a igualdade

$$
\sum_{\omega \in \Omega_{\Lambda}} e^{-\beta \mathcal{H}_{\Lambda, h}(\omega)}=2^{|\Lambda|} \sum_{\partial \mathbf{n}=\emptyset} w(\mathbf{n}) .
$$

Podemos finalmente concluir que (4.5) e (4.6) estão provados.

O método das representações por correntes é muito útil, pois existe uma identidade combinatória importantíssima sobre ele, que permite co- 
mutar fontes em sistemas duplicados de correntes. Um caso particular deste fato é o famoso Lema da Comutação em Inglês Switching Lemma. Antes de apresentar o enunciado deste lema precisamos introduzir mais algumas definições.

Sítios conectados em n. Diremos que dois sítios $x, y$ ( $y$ pode ser o sítio fantasma) estão conectados na configuração $\mathbf{n}$, denotaremos isto por $x \stackrel{\mathbf{n}}{\longleftrightarrow} y$, se existe um caminho no grafo $\left(\Lambda^{g}, \overline{\mathcal{E}_{\Lambda}}\right)$ de $x$ para $y$ tais que para toda aresta deste caminho temos $n_{b} \neq 0$.

Aglomerado de arestas de um sítio. O conjunto de arestas com pelo menos um sítio conectado a $x$, será chamado de aglomerado de arestas do sítio $x$ na configuração $\mathbf{n}$, isto é,

$$
C_{\mathbf{n}}(x)=\{\{y, z\}: y \stackrel{\mathbf{n}}{\longleftrightarrow} x \text { ou } z \stackrel{\mathbf{n}}{\longleftrightarrow} x\} \cup\{\{y, g\}: y \stackrel{\mathbf{n}}{\longleftrightarrow} x\} .
$$

Observamos que $C_{\mathbf{n}}(g)$ contém pelo menos todas as " $h$-arestas" com fluxo não nulo.

Lema 4.1.3 (Lema da Comutação). 1 Seja A um conjunto de sítios de uma rede aumentada, $\{x, y\}$ um par de sítios (um dos quais pode ser $g$ ), e $f$ uma função definida em configurações de correntes. Então

$$
\sum_{\substack{\partial \boldsymbol{n}_{1}=A \\ \partial \mathbf{n}_{2}=\{x, y\}}} w\left(\boldsymbol{n}_{1}\right) w\left(\boldsymbol{n}_{2}\right) f\left(\boldsymbol{n}_{1}+\boldsymbol{n}_{2}\right)=\sum_{\substack{\partial \mathbf{n}_{1}=A \Delta\{x, y\} \\ \partial \mathbf{n}_{2}=\emptyset}} w\left(\boldsymbol{n}_{1}\right) w\left(\boldsymbol{n}_{2}\right) f\left(\boldsymbol{n}_{1}+\boldsymbol{n}_{2}\right) 1_{\left\{x \stackrel{\boldsymbol{n}_{1}+\boldsymbol{n}_{2}}{\longrightarrow} y\right\}},
$$

onde $\left(\boldsymbol{n}_{1}+\boldsymbol{n}_{2}\right)_{b}=\left(\boldsymbol{n}_{1}\right)_{b}+\left(\boldsymbol{n}_{2}\right)_{b}$.

Demonstração. Veja [22].

Este lema é muito útil para lidar com funções de correlações truncadas 2 , onde frequentemente precisamos levar em conta delicados cancelamentos vindos de contribuições com sinais distintos. Dois exemplos de aplicação deste lema, que serão explorados nos capítulos seguintes, são apresentados abaixo em forma das seguintes proposições.

\footnotetext{
${ }^{1} A \Delta B=(A \backslash B) \cup(B \backslash A) ;$

${ }^{2}\left\langle\sigma_{A} ; \sigma_{B}\right\rangle=\left\langle\sigma_{A} \sigma_{B}\right\rangle-\left\langle\sigma_{A}\right\rangle\left\langle\sigma_{B}\right\rangle$.
} 
Proposição 4.1.1. Seja $\Lambda$ uma rede finita contendo os sítios $x$ e 0 , então

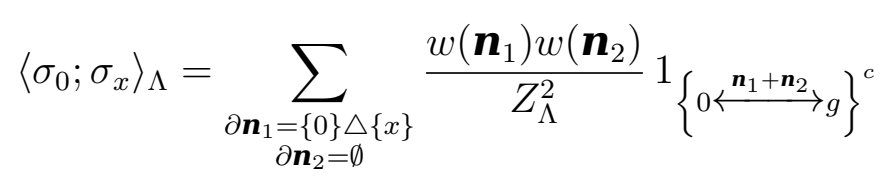

Demonstração. Aplicando respectivamente as identidades 4.5 e 4.6 temos

$$
\begin{aligned}
& \left\langle\sigma_{0} ; \sigma_{x}\right\rangle_{\Lambda}=\left\langle\sigma_{0} \sigma_{x}\right\rangle_{\Lambda}-\left\langle\sigma_{0}\right\rangle_{\Lambda}\left\langle\sigma_{x}\right\rangle_{\Lambda} \\
& =\frac{\sum_{\partial \mathbf{n}=\{0, x\}} w(\mathbf{n})}{Z_{\Lambda}}-\frac{\sum_{\partial \mathbf{n}=\{0, g\}} w(\mathbf{n}) \sum_{\partial \mathbf{n}=\{x, g\}} w(\mathbf{n})}{Z_{\Lambda}^{2}} \\
& =\frac{\sum_{\partial \mathbf{n}_{1}=\{0, x\}} w\left(\mathbf{n}_{1}\right) \sum_{\partial \mathbf{n}_{2}=\emptyset} w\left(\mathbf{n}_{2}\right)-\sum_{\partial \mathbf{n}_{1}=\{0, g\}} w\left(\mathbf{n}_{1}\right) \sum_{\partial \mathbf{n}_{2}=\{x, g\}} w\left(\mathbf{n}_{2}\right)}{Z_{\Lambda}^{2}}
\end{aligned}
$$

onde na última igualdade usamos o Lema 4.1.1. Agora, aplicando o Lema da Comutação na segunda parcela do numerador, ficamos com

$$
\begin{aligned}
& \left.\left\langle\sigma_{0} ; \sigma_{x}\right\rangle_{\Lambda}=\frac{1}{Z_{\Lambda}^{2}}\left(\sum_{\substack{\partial \mathbf{n}_{1}=\{0, x\} \\
\partial \mathbf{n}_{2}=\emptyset}} w\left(\mathbf{n}_{1}\right) w\left(\mathbf{n}_{2}\right)-\sum_{\substack{\partial \mathbf{n}_{1}=\{0\} \triangle\{x\} \\
\partial \mathbf{n}_{2}=\emptyset}} w\left(\mathbf{n}_{1}\right) w\left(\mathbf{n}_{2}\right) 1_{\left\{0 \stackrel{\mathbf{n}_{1}+\mathbf{n}_{2}}{\longrightarrow} g\right.}\right\}\right) \\
& =\frac{1}{Z_{\Lambda}^{2}} \sum_{\substack{\partial \mathbf{n}_{1}=\{0\} \triangle\{x\} \\
\partial \mathbf{n}_{2}=\emptyset}} w\left(\mathbf{n}_{1}\right) w\left(\mathbf{n}_{2}\right)\left[1-1_{\left\{0 \stackrel{\mathbf{n}_{1}+\mathbf{n}_{2}}{\longrightarrow} g\right\}}^{\stackrel{1}{\longrightarrow}}\right] \\
& =\sum_{\substack{\partial \mathbf{n}_{1}=\{0\} \triangle\{x\} \\
\partial \mathbf{n}_{2}=\emptyset}} \frac{w\left(\mathbf{n}_{1}\right) w\left(\mathbf{n}_{2}\right)}{Z_{\Lambda}^{2}} 1_{\left\{0 \stackrel{\mathbf{n}_{1}+\mathbf{n}_{2}}{\longrightarrow} g\right\}^{c}}
\end{aligned}
$$

Proposição 4.1.2. Seja $\Lambda$ uma rede finita contendo os sítios $u, v$ e 0 , então

$$
\left.\left\langle\sigma_{0} ; \sigma_{u} \sigma_{v}\right\rangle_{\Lambda}=\sum_{\substack{\partial \mathbf{n}_{1}=\{0, g\} \Delta\{u\} \Delta\{v\} \\ \partial \mathbf{n}_{2}=\emptyset}} \frac{w\left(\boldsymbol{n}_{1}\right) w\left(\boldsymbol{n}_{2}\right)}{Z_{\Lambda}^{2}} 1_{\left\{0 \stackrel{\boldsymbol{n}_{1}+\boldsymbol{n}_{2}}{\rightleftarrows} g\right.}^{\stackrel{c}{c}}\right\}^{c}
$$


Demonstração. De 4.5, 4.6 e do Lema 4.1.1 segue que

$$
\begin{aligned}
& \left\langle\sigma_{0} ; \sigma_{u} \sigma_{v}\right\rangle_{\Lambda}=\left\langle\sigma_{0} \sigma_{u} \sigma_{v}\right\rangle_{\Lambda}-\left\langle\sigma_{0}\right\rangle_{\Lambda}\left\langle\sigma_{u} \sigma_{v}\right\rangle_{\Lambda} \\
& =\frac{\sum_{\partial \mathbf{n}=\{0, u, v, g\}} w(\mathbf{n})}{Z_{\Lambda}}-\frac{\sum_{\partial \mathbf{n}=\{0, g\}} w(\mathbf{n}) \sum_{\partial \mathbf{n}=\{u, v\}} w(\mathbf{n})}{Z_{\Lambda}^{2}} \\
& =\frac{\sum_{\partial \mathbf{n}_{1}=\{0, u, v, g\}} w\left(\mathbf{n}_{1}\right) \sum_{\partial \mathbf{n}_{2}=\emptyset} w\left(\mathbf{n}_{2}\right)-\sum_{\partial \mathbf{n}_{1}=\{u, v\}} w\left(\mathbf{n}_{1}\right) \sum_{\partial \mathbf{n}_{2}=\{0, g\}} w\left(\mathbf{n}_{2}\right)}{Z_{\Lambda}^{2}} .
\end{aligned}
$$

Aplicando Lema da Comutação na segunda parcela, podemos reescrever a expressão acima como segue

$$
\left.\sum_{\substack{\partial \mathbf{n}_{1}=\{0, u, v, g\} \\ \partial \mathbf{n}_{2}=\emptyset}} \frac{w\left(\mathbf{n}_{1}\right) w\left(\mathbf{n}_{2}\right)}{Z_{\Lambda}^{2}}-\sum_{\substack{\partial \mathbf{n}_{1}=\{0, g\} \triangleleft\{u, v\} \\ \partial \mathbf{n}_{2}=\emptyset}} \frac{w\left(\mathbf{n}_{1}\right) w\left(\mathbf{n}_{2}\right)}{Z_{\Lambda}^{2}} 1_{\left\{0 \stackrel{\mathbf{n}_{1}+\mathbf{n}_{2}}{\longleftrightarrow} g\right\}}\right\}
$$

Subtraindo os dois somatórios e usando propriedades elementares da diferença simétrica temos finalmente que

$$
\left\langle\sigma_{0} ; \sigma_{u} \sigma_{v}\right\rangle_{\Lambda}=\sum_{\substack{\partial \mathbf{n}_{1}=\{0, g\} \Delta\{u\} \triangleleft\{v\} \\ \partial \mathbf{n}_{2}=\emptyset}} \frac{w\left(\mathbf{n}_{1}\right) w\left(\mathbf{n}_{2}\right)}{Z_{\Lambda}^{2}} 1_{\left\{0 \stackrel{\mathbf{n}_{1}+\mathbf{n}_{2}}{\longrightarrow} g\right\}^{c}}
$$

Na próxima seção vamos utilizar estas duas fórmulas deduzidas acima para ajudar na análise do comportamento das seguintes funções

$$
\chi_{\Lambda}(\beta, h)=\frac{1}{\beta} \frac{\partial M_{\Lambda}}{\partial h}(\beta, h)=\sum_{x \in \Lambda}\left\langle\sigma_{0} ; \sigma_{x}\right\rangle_{\Lambda}
$$

e

$$
\frac{\partial M_{\Lambda}}{\partial \beta}(\beta, h)=\sum_{x, y \in \Lambda} J(x-y)\left\langle\sigma_{0} ; \sigma_{x} \sigma_{y}\right\rangle_{\Lambda} .
$$

As duas igualdades acima podem ser obtidas diretamente das definições de $M_{\Lambda}$ e $\chi_{\Lambda}$. 


\subsubsection{Condicionamento Sobre Aglomerados}

Diferentemente do Lema de Comutação que permite trabalhar com identidades combinatórias, a técnica de condicionamento sobre aglomerados que vamos introduzir a seguir, permite reescrever algumas expressões em uma forma que bastante conveniente.

Para facilitar a compreensão deste novo conceito vamos inicialmente considerar a seguinte configuração de correntes esboçada na figura abaixo:

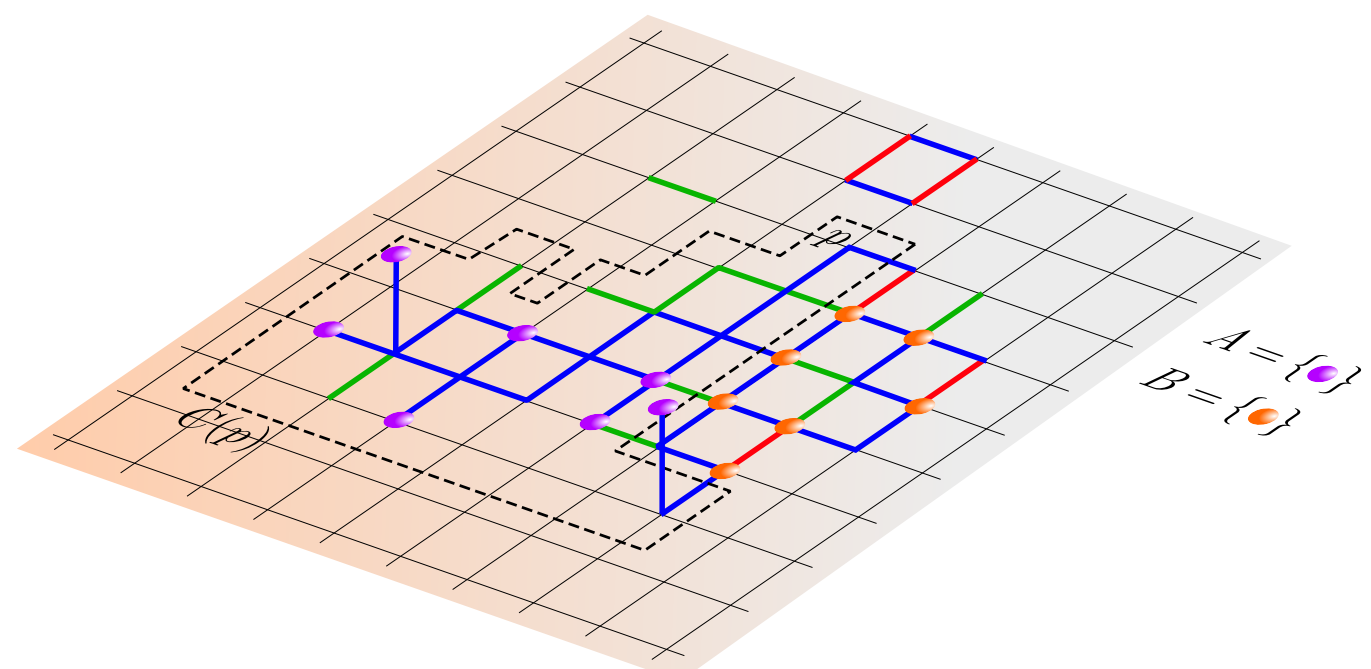

Figura 4.1: As arestas azuis representam fluxos de valor 1, enquanto que as verdes são as que tem fluxo de valor 2 , e as vermelhas são de valor 3 . A é o conjunto de fontes de cor rocha, e $B$ é o conjunto de fontes de cor laranja. As arestas que estão inclinadas representam arestas ligando um sítio da rede ao sítio fantasma (que é único, mas aqui o desenhamos em multiplicidade para facilitar a visualização)

Nesta figura $A$ e $B$ são conjuntos de fontes e $p$ é um sitio arbitrário em $\Lambda^{g}$. A ideia por traz do condicionamento sobre aglomerados é considerar que as correntes $\mathbf{n}=\left\{n_{b}\right\}_{b \in \overline{\mathcal{E}_{\Lambda}}}$ em cada aresta são variáveis aleatórias independentes e olhar para as configurações de correntes aleatórias sujeitas à condição de que $p \in C_{\mathbf{n}}(a)$ para todo $a \in A$, mas $p \notin C_{\mathbf{n}}(b)$ para todo $b \in B$; a linha entrecortada representa esta condição, neste momento denotaremos essa linha por $C(p)$. Voltando a nossa figura estamos interessados em considerar configurações de correntes cujas as arestas que saem da linha $C(p)$ tenham fluxo nulo. Esta técnica consiste em condicionar sobre $C(p)$, ou seja, em somar livremente e independentemente sobre configurações correntes no interior e no exterior do aglomerado $C(p)$. 
Lema 4.1.4. Para todo $\Lambda$ finito, $u, v \in \Lambda$ temos que

$$
\left\langle\sigma_{0} ; \sigma_{u} \sigma_{v}\right\rangle_{\Lambda}=\sum_{\substack{\partial \boldsymbol{n}_{1}=\{0\} \Delta\{u\} \\ \partial \boldsymbol{n}_{2}=\emptyset}} \frac{w\left(\boldsymbol{n}_{1}\right) w\left(\boldsymbol{n}_{2}\right)}{Z_{\Lambda}^{2}}\left\langle\sigma_{v}\right\rangle_{[C(0)]^{c}}+\sum_{\substack{\partial \boldsymbol{n}_{1}=\{0\} \Delta\{u\} \\ \partial \mathbf{n}_{2}=\emptyset}} \frac{w\left(\boldsymbol{n}_{1}\right) w\left(\boldsymbol{n}_{2}\right)}{Z_{\Lambda}^{2}}\left\langle\sigma_{v}\right\rangle_{[C(0)] c},
$$

onde $[C(0)]^{c}$ é uma notação reduzida de $\left[C_{\boldsymbol{n}_{1}+\boldsymbol{n}_{2}}(0)\right]^{c}$ e sendo A um conjunto de arestas, o subíndice $A$, em $Z_{A} e\langle\cdot\rangle_{A}$ indicam que as constantes de acoplamento são todas zero para todas as arestas que não pertencem a A.

Demonstração. Da equação (4.10) temos que

$$
\left.\left\langle\sigma_{0} ; \sigma_{u} \sigma_{v}\right\rangle_{\Lambda}=\sum_{\substack{\partial \mathbf{n}_{1}=\{0, g\} \Delta\{u\} \Delta\{v\} \\ \partial \mathbf{n}_{2}=\emptyset}} \frac{w\left(\mathbf{n}_{1}\right) w\left(\mathbf{n}_{2}\right)}{Z_{\Lambda}^{2}} 1_{\left\{0 \stackrel{\mathbf{n}_{1}+\mathbf{n}_{2}}{\longrightarrow} g\right.}\right\}^{c} .
$$

Pelo Lema do Aperto de Mãos, sabemos que o número de vértices de grau ímpar em cada componente conexa deve ser par, assim podemos verificar que os termos que dão contribuição não-nula, na soma acima, são aqueles em que acontece uma das duas alternativas abaixo:

i) 0 e $u$ estão no mesmo aglomerado, digamos $A$ e $v$ e $g$ estão no mesmo aglomerado $B$ e estes aglomerados são disjuntos;

ii) 0 e $v$ estão no mesmo algomerado, digamos $C$ e $u$ e $g$ estão no mesmo aglomerado $D$ e estes aglomerados são disjuntos;

Desta forma podemos representar os somatórios que aparecem acima como mostra a figura:

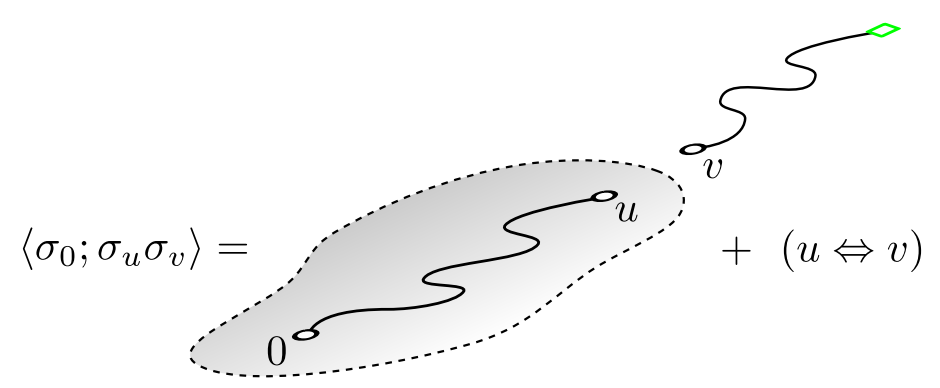

Figura 4.2: Representação das parcela de (4.10). A notação $(u \Leftrightarrow$ $v$ ) significa o desenho a esquerda deveria ser reproduzido com $v$ no lugar de $u$. O sítio verde que aparece ligado a $v$ é o sítio fantasma. 
Quando condicionamos sobre o aglomerado $C_{\mathbf{n}_{1}+\mathbf{n}_{2}}(0)$, as somas sobre as configurações de correntes dentro e fora do cluster são condicionalmente independentes. A soma no exterior nos fornece o valor esperado de $\sigma_{u}$ (ou $\sigma_{v}$ ) no sistema que sofreu a retirada dos elos de $C_{\mathbf{n}_{1}+\mathbf{n}_{2}}(0)$, multiplicado pela função de partição deste sistema reduzido. Usando esta função para "retirar o condicionamento" da soma obtemos a identidade desejada.

Lema 4.1.5. Para todo $\Lambda$ finito e $x \in \Lambda$ temos

$$
\left\langle\sigma_{0} ; \sigma_{x}\right\rangle_{\Lambda}=\sum_{\substack{\partial \boldsymbol{n}_{1}=\emptyset \\ \partial \boldsymbol{n}_{2}=\emptyset}} \frac{w\left(\boldsymbol{n}_{1}\right) w\left(\boldsymbol{n}_{2}\right)}{Z_{\Lambda}^{2}}\left\langle\sigma_{0} \sigma_{x}\right\rangle_{[C(g)]^{c}} 1_{\left\{0 \stackrel{\boldsymbol{n}_{1}+\boldsymbol{n}_{2}}{\longrightarrow} g\right\}^{c}}
$$

Demonstração. A prova é totalmente análoga a do lema anterior, basta usar a técnica de condicionamento sobre aglomerados.

\subsubsection{Representações por Passeios Aleatórios}

Esta representação permite ver a representação por correntes aleatórias por meio dos chamados backbones (espinha dorsal). Para fazer isso definiremos uma transformação $\Omega_{B}(\mathbf{n})$, chamada $B$-backbone de $\mathbf{n}$, a qual para cada configuração de correntes $\mathbf{n}$ com fontes $\partial \mathbf{n}=B$, associamos uma sequência bem definida de caminhos $\omega$ ligando os sítios de $B$ em pares. Isto nos permitirá reescrever as correlações (4.5) e (4.6) da seguinte maneira:

$$
\begin{aligned}
\left\langle\sigma_{A}\right\rangle=\sum_{\partial \omega=A} \rho(\omega), & \text { se }|A| \text { é par; } \\
\left\langle\sigma_{A}\right\rangle=\sum_{\partial \omega=A \cup\{g\}} \rho(\omega), & \text { se }|A| \text { é ímpar, }
\end{aligned}
$$

onde

$$
\rho(\omega)=\sum_{\partial \mathbf{n}=\partial \omega} \frac{w(\mathbf{n})}{Z} 1_{\left\{\Omega_{\partial \omega}(n)=\omega\right\}}
$$

e $\partial w$ é (análogo natural) o conjunto de sítios em $\Lambda^{g}$ que são visitados por um número ímpar de passos de $\omega$.

Passamos agora para a definição formal do backbone de uma configuração de correntes. A primeiro passo é considerar uma ordem total na rede $\mathbb{Z}^{d}$. Assim cada um de seus subconjuntos tem um menor sítio ou "primeiro" sítio. Esta ordem pode ser, por exemplo, a ordem lexicográfica para modelos de alcance finito. Note que a ordem pode ser estendida à rede estendida declarando ao sítio fantasma como sendo o menor ele- 
mento de todos.

Vamos precisar também das seguintes definições:

Passo. Uma passo de $x$ para $y$ é uma aresta ordenada $(x, y)$.

Para cada $x \in \Lambda$ escolhemos uma ordem para o conjunto dos passos que começam em $x$ de forma que um passo da forma $(x, g)$ seja sempre o menor de todos. Para este ordenamento podemos usar a ordem total que consideramos, acima, nos vértices da rede estendida.

Aresta cancelada. Para cada passo $(x, y)$ (y pode ser o sítio fantasma), associamos um conjunto de arestas canceladas formado pela aresta $\{x, y\}$ e por todas as arestas correspondentes a passos que saem de $x$ que são anteriores a $(x, y)$.

Sequência consistente. Uma sequência de passos é dita consistente se nenhum passo da sequência usa uma aresta cancelada previamente por outro passo.

Caminho ou passeio. Um caminho de $u$ para $v$ é uma sequência de pas$\operatorname{sos}\left\{\left(x_{i}, x_{i+1}\right), i=0, \ldots, n\right\} \operatorname{com} x_{0}=u$ e $x_{n+1}=v$.

Fontes de uma sequência de passos. Dada uma sequência de passos $\omega$ um sítio em $x \in \Lambda^{g}$ é uma fonte da sequência de passos $\omega$ se $x$ pertence a um número ímpar de passos da sequência $\omega$. O conjunto de todas as fontes de uma sequência de passos $\omega$ é denotado por $\partial \omega$.

Backbone $\Omega_{B}(\mathbf{n})$. Para uma configuração correntes $\mathbf{n}$ com $\partial \mathbf{n}=B$, o backbone $\Omega_{B}(\mathbf{n})$ consiste de um conjunto de caminhos com as seguintes propriedades:

1. O primeiro caminho $\omega_{1}$, inicia-se no "primeiro" sítio $u_{1}$, do conjunto $B \backslash\{g\}$ e termina quando alcançar algum outro sítio de $B$ ou o sítio fantasma (mesmo que $g$ não esteja em $B$ ). Seu primeiro passo é $\left(u_{1}, u_{2}\right)$, onde $u_{2}$ é o menor sítio $v$ que tal que $n_{\left\{u_{1}, v\right\}}$ é ímpar.

2. Até que o caminho pare, cada passo do caminho $\left(u_{i}, u_{i+1}\right)$ é o menor de todos os passos $\left(u_{i}, v\right)$, que não usam arestas canceladas por passos anteriores e além do mais $n_{\left\{u_{i}, v\right\}}$ é ímpar. 
3. Quando $\omega_{1}$ para, nós escolhemos o primeiro dos sítios de $B$ que não foi visitado por $\omega_{1}$, e repetimos o processo com todas as arestas que não foram canceladas por $\omega_{1}$. Com isto temos um segundo caminho $\omega_{2}$.

4. Continuamos assim até terminar com todos os elementos de $B$. Ao fim de tudo, nós obtemos caminhos $\omega_{1}, \omega_{2}, \cdots, \omega_{s}(s \leq|B|)$ percorrido nessa ordem. É a este conjunto de caminhos que nós chamamos $B$ backbone e o denotaremos por $\Omega_{B}(\mathbf{n})=\omega_{1} \circ \omega_{2} \circ \cdots \circ \omega_{s}$.

Agora, conheceremos as propriedades de $\rho(\omega)$, que são:

1. Para cada decomposição de uma sequência consistente de caminhos $\omega$ em duas sequências $\omega=\omega_{1} \circ \omega_{2}$, temos

$$
\rho\left(\omega_{1} \circ \omega_{2}\right)=\rho\left(\omega_{1}\right) \rho_{\omega_{1}^{c}}\left(\omega_{2}\right)
$$

onde $\rho_{A^{c}}(\omega)$ é o peso para o sistema no qual todas as constantes de acoplamento de as arestas em $A$ são zero.

2. Para um conjunto de arestas $A, \operatorname{com} \omega \cap A=\emptyset$ temos

$$
\rho(\omega) \leq \rho_{A^{c}}(\omega)
$$

Estas dois propriedades anteriores podem ser vistas com mais detalhe em [5].

Para fazer deixar destacado o emparelhamento das fontes induzido pela aplicação de "backbone", usamos a notação $\omega_{i}: x \rightarrow y$. para indicar que $\omega_{i}$ é um caminho que inicia em $x$, termina em $y$ e visita $y$ uma única vez.

Exemplo 4.1.1. No modelo de Ising para todo $\Lambda$ finito contendo a origem temos

$$
\left\langle\sigma_{0}\right\rangle=\sum_{\omega: 0 \rightarrow g} \rho(\omega) .
$$

Supondo que o modelo tem campo nulo e $x, y \in \Lambda$ então é válida a seguinte identidade

$$
\left\langle\sigma_{x} \sigma_{y}\right\rangle=\sum_{\omega: x \rightarrow y} \rho(\omega)
$$


Capítulo 4. Representações por Correntes e Passeios aleatórios 


\section{Capítulo 5}

\section{Prova da Principal Desigualdade Diferencial}

Neste capítulo nosso objetivo é demostrar o Teorema 5.0.1, que para modelos de spin de Ising nos fornece a principal desigualdade diferencial parcial. Para deduzir essa desigualdade usaremos as expansões por correntes aleatórias e por caminhos aleatórios. A principal referência usada neste capítulo é [3].

Observação 5.0.2. Os lemas e teoremas neste capitulo e no seguinte serão expressadas no limite termodinâmico mas suas provas as faremos a volume finito. Quando fazemos as provas a volume finito as medias $\langle\cdot\rangle \mathrm{se-}$ rão consideradas a volume finito e as vezes não se indicara na sua notação o volume.

Teorema 5.0.1 (Teorema 2 (i)). Para um modelo de spins de Ising ferromagnético invariante por translações em $(-L, L]^{d}$, a magnetização obedece a seguinte D.D.P. (Desigualdade Diferencial Parcial)

$$
M(\beta, h) \leq \tanh (\beta h) \chi(\beta, h)+M^{3}(\beta, h)+M^{2}(\beta, h) \beta \frac{\partial M}{\partial \beta}(\beta, h),
$$

onde $M$ e $\chi$ são os limites termodinâmicos da magnetização e susceptibilidade a volume finito, respectivamente.

Vamos fazer alguns preparativos antes de passar para o prova do Teorema. Primeiro vamos relembrar que no capítulo anterior deduzimos a seguinte identidade

$$
\left\langle\sigma_{0}\right\rangle_{\Lambda}=\sum_{\partial \mathbf{n}_{1}=\{0, g\}} Z_{\Lambda}^{-1} \omega\left(\mathbf{n}_{1}\right)
$$


Vamos introduzir nesta igualdade, no lado direito, duas somas "falsas", como mostrado abaixo

$$
\left\langle\sigma_{0}\right\rangle_{\Lambda}=\sum_{\substack{\partial \mathbf{n}_{1}=\{0, g\} \\ \partial \mathbf{n}_{2}=\emptyset \\ \partial \mathbf{n}_{3}=\emptyset}} \frac{\omega\left(\mathbf{n}_{1}\right) \omega\left(\mathbf{n}_{2}\right) \omega\left(\mathbf{n}_{3}\right)}{Z_{\Lambda}^{3}} .
$$

Estamos interessados nos pontos da rede que estão no backbone $\Omega_{\{0, g\}}\left(\mathbf{n}_{1}\right)$, e são conectados a $g$ na configuração de correntes $\mathbf{n}_{2}+\mathbf{n}_{3}$. Vamos classificar as configurações de correntes pelo primeiro sítio com tal propriedade (primeiro significa com respeito a ordem natural induzida pelo backbone $\left(\Omega_{\{0, g\}}\left(\mathbf{n}_{1}\right)\right)$. Este procedimento nos leva a considerar três casos:

1. Não existe tal sítio;

2. O primeiro sítio é 0 ;

3. O primeiro de tais sítios é algum $v \neq 0$.

No terceiro caso, o primeiro passo $(u, v)$ a atingir $v$ divide o backbone $\Omega_{\{0, g\}}\left(\mathbf{n}_{1}\right)$ em duas partes. A primeira é aquela onde 0 e $u$ estão conectados, sem serem conectados por $g$, com respeito a configuração de correntes $\mathbf{n}_{2}+\mathbf{n}_{3}$.

Baseado, nas ideias acima, vamos decompor $M=\left\langle\sigma_{0}\right\rangle_{\Lambda}$ da seguinte maneira:

$$
M=T+R_{0}+\sum_{u} \sum_{v \neq 0} R_{u v}
$$

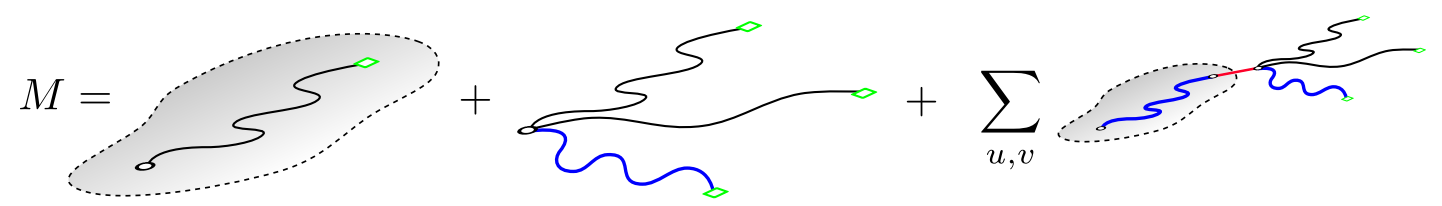

Figura 5.1: A linha solida de cor azul é o backbone $\Omega_{\{0, g\}}\left(\mathbf{n}_{1}\right)$, a outra linha solida representa conexões devidas a $\mathbf{n}_{2}+\mathbf{n}_{3}$ e a linha entrecortada representa de $C_{\mathbf{n}_{2}+\mathbf{n}_{3}}(g)$.

onde

$$
\begin{array}{r}
T=\sum_{\substack{\partial \mathbf{n}_{1}=\{0, g\} \\
\partial \mathbf{n}_{2}=\emptyset \\
\partial \mathbf{n}_{3}=\emptyset}} \frac{\omega\left(\mathbf{n}_{1}\right) \omega\left(\mathbf{n}_{2}\right) \omega\left(\mathbf{n}_{3}\right)}{Z_{\Lambda}^{3}} 1_{\left\{\begin{array}{c}
\text { nenhuma das arestas de } \left.\Omega_{\{0, g\}}\left(\mathbf{n}_{1}\right)\right\} \\
\text { está em } \mathbf{n}_{\mathbf{n}_{2}+\mathbf{n}_{3}(g)}
\end{array},\right.} \\
R_{0}=\sum_{\substack{\partial \mathbf{n}_{1}=\{0, g\} \\
\partial \mathbf{n}_{2}=\emptyset \\
\partial \mathbf{n}_{3}=\emptyset}} \frac{\omega\left(\mathbf{n}_{1}\right) \omega\left(\mathbf{n}_{2}\right) \omega\left(\mathbf{n}_{3}\right)}{Z_{\Lambda}^{3}} 1_{\left\{\mathbf{n}_{2}+\mathbf{n}_{3}: 0 \leftrightarrow g\right\}} \text { (Lema da Comutação) }\left\langle\sigma_{0}\right\rangle_{\Lambda}^{3}
\end{array}
$$




\section{5}

e

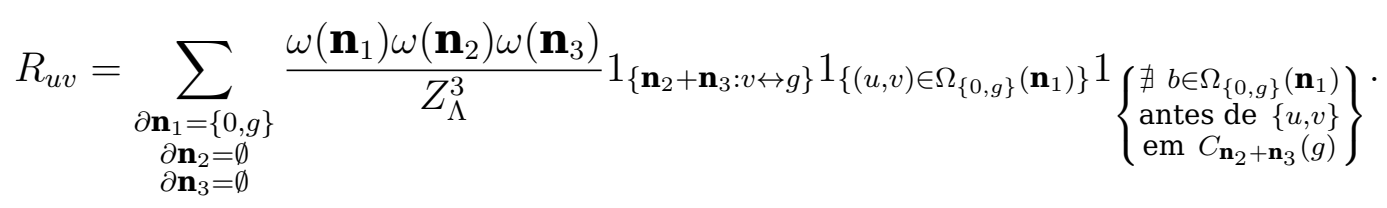

Notamos que cada sítio conectado a $g$ via $\mathbf{n}_{2}+\mathbf{n}_{3}$ deve estar duplamente conectado, já que $\partial\left(\mathbf{n}_{2}+\mathbf{n}_{3}\right)=\emptyset$.

Nosso objetivo agora é limitar $T, R_{0}$ e $R_{u, v}$ por quantidades físicas. Para conseguir isto usaremos as duas técnicas: a de caminhos aleatórios e a de correntes aleatórias.

Lema 5.0.6.

$$
T \leq \tanh (\beta h) \frac{\partial M}{\partial(\beta h)}
$$

Demonstração. Vamos analisar o lado direito da equação (5.5). Notamos que no último passo $(x, g)$ do backbone $\mathbf{n}_{1}$ o fluxo $n_{1\{x, g\}}$ é ímpar, porque $g$ é fonte.

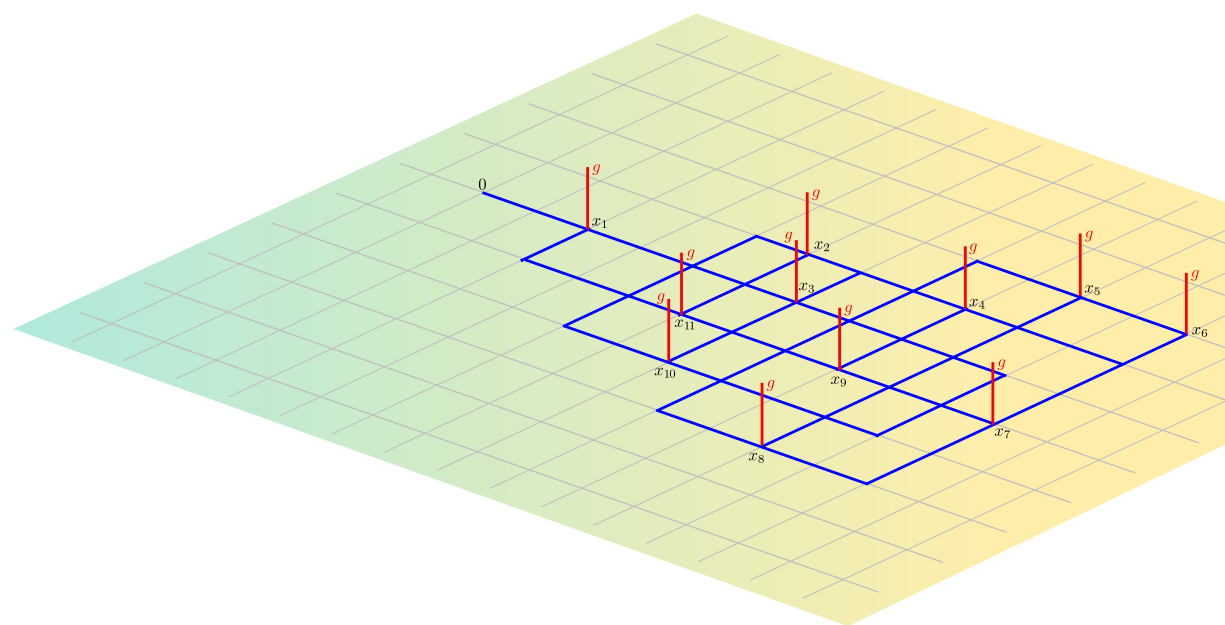

Figura 5.2: Todos os fluxos deste grafo tem valor igual a 1. Os fluxos das arestas que unem o sítio fantasma com os outros sítios vão mudar de ímpar para par.

Para cada sítio $x$ estimamos a correspondente contribuição a $T$ considerando o conjunto de correntes obtidas mudando $n_{1\{x, g\}}$ ímpar para um fluxo par. Veremos agora, que a soma considerando os $n_{1\{x, g\}}$ ímpares é equivalente à soma considerando os $n_{1\{x, g\}}$ pares mas multiplicada por um 
fator de $\tanh (\beta h)$.

Para a nova soma, notamos que as configurações tem como fontes os sítios $\{0, x\}$. Com a condição, como na soma anterior, que backbone $\mathbf{n}_{1}$ alcança $x$ antes de alcançar $C_{\mathbf{n}_{1}+\mathbf{n}_{2}}(g)$. Portanto

$$
\begin{aligned}
T & =\tanh (\beta h) \times \\
& \times \sum_{x \in \Lambda} \sum_{\substack{\partial \mathbf{n}_{1}=\{0\} \\
\text { ond } \\
\partial \mathbf{n}_{3}=\emptyset}} \frac{\omega\left(\mathbf{n}_{1}\right) \omega\left(\mathbf{n}_{2}\right) \omega\left(\mathbf{n}_{3}\right)}{Z_{\Lambda}^{3}} 1_{\left\{\Omega_{\{0, x\}}\left(\mathbf{n}_{1}\right) \cap C_{\mathbf{n}_{2}+\mathbf{n}_{3}}(g)=\emptyset\right\}} 1_{\left\{n_{1\{x, g\}} \mathrm{épar}\right\}} 1_{\left\{\mathbf{n}_{2}+\mathbf{n}_{3}: 0 \leftrightarrow g\right\}}
\end{aligned}
$$

Usando a representação de caminhos aleatórios para $\mathbf{n}_{1}$ temos que:

$$
\sum_{\partial \mathbf{n}_{1}=\{0\} \Delta\{x\}} Z_{\Lambda}^{-1} w\left(\mathbf{n}_{1}\right) 1_{\left\{\Omega_{\{0, x\}}\left(\mathbf{n}_{1}\right) \cap C_{\left.\mathbf{n}_{2}+\mathbf{n}_{3}(g)=\emptyset\right\}}\right.}=\sum_{\omega: 0 \rightarrow x} \rho(\omega) 1_{\left\{\Omega_{\{0, x\}}\left(\mathbf{n}_{1}\right) \cap C_{\mathbf{n}_{2}+\mathbf{n}_{3}}(g)=\emptyset\right\}},
$$

e como a função indicadora é limitada por 1, segue que:

$$
T \leq \tanh (\beta h) \sum_{x \in \Lambda} \sum_{\substack{\mathbf{n}_{2}=\emptyset \\ \partial \mathbf{n}_{3}=\emptyset}} Z_{\Lambda}^{-2} \omega\left(\mathbf{n}_{2}\right) \omega\left(\mathbf{n}_{3}\right) 1_{\left\{\mathbf{n}_{2}+\mathbf{n}_{3}: 0 \leftrightarrow g\right\}} \sum_{\omega: 0 \rightarrow x} \rho(\omega) 1_{\left\{\Omega_{\{0, x\}}\left(\mathbf{n}_{1}\right) \cap C_{\mathbf{n}_{2}+\mathbf{n}_{3}}(g)=\emptyset\right\}} .
$$

Por (4.19) a expressão acima é limitada superiormente por

$$
\tanh (\beta h) \sum_{x \in \Lambda} \sum_{\substack{\partial \mathbf{n}_{2}=\emptyset \\ \partial \mathbf{n}_{3}=\emptyset}} \frac{\omega\left(\mathbf{n}_{2}\right) \omega\left(\mathbf{n}_{3}\right)}{Z_{\Lambda}^{2}} 1_{\left\{\mathbf{n}_{2}+\mathbf{n}_{3}: 0 \leftrightarrow g\right\}} \sum_{\omega: 0 \rightarrow x} \rho_{[C(g)]^{c}}(\omega) 1_{\left\{\Omega_{\{0, x\}}\left(\mathbf{n}_{1}\right) \cap C_{\left.\mathbf{n}_{2}+\mathbf{n}_{3}(g)=\emptyset\right\}}\right.}
$$

continuando a estimativa, agora usando (4.21) e (4.14) obtemos

$$
\begin{aligned}
T & \leq \tanh (\beta h) \sum_{x \in \Lambda} \sum_{\substack{\mathbf{n}_{2}=\emptyset \\
\partial \mathbf{n}_{3}=\emptyset}} \frac{\omega\left(\mathbf{n}_{2}\right) \omega\left(\mathbf{n}_{3}\right)}{Z_{\Lambda}^{2}}\left\langle\sigma_{0} \sigma_{x}\right\rangle_{[C(g)]^{c}} 1_{\left\{\mathbf{n}_{2}+\mathbf{n}_{3}: 0 \leftrightarrow g\right\}} \\
& =\tanh (\beta h) \sum_{x \in \Lambda}\left\langle\sigma_{0} ; \sigma_{x}\right\rangle_{\Lambda} .
\end{aligned}
$$

Lembrando de (4.11) e usando a estimativa acima, temos finalmente que

$$
T \leq \tanh (\beta h) \sum_{x \in \Lambda}\left\langle\sigma_{0} ; \sigma_{x}\right\rangle_{\Lambda}=\tanh (\beta h) \frac{\partial M_{\Lambda}}{\partial(\beta h)}
$$

e assim o lema está demonstrado. 
Lema 5.0.7.

$$
R_{0}=\left\langle\sigma_{0}\right\rangle^{3} .
$$

Demonstração.

$$
R_{0}=\sum_{\partial \mathbf{n}_{1}=\{0, g\}} Z_{\Lambda}^{-1} w\left(\mathbf{n}_{1}\right) \sum_{\substack{\partial \mathbf{n}_{2}=\emptyset \\ \partial \mathbf{n}_{3}=\emptyset}} Z_{\Lambda}^{-2} \omega\left(\mathbf{n}_{2}\right) \omega\left(\mathbf{n}_{3}\right) 1_{\left\{\mathbf{n}_{2}+\mathbf{n}_{3}: 0 \leftrightarrow g\right\}} .
$$

Aplicando o Lema de Comutação ( $\mathbf{n}_{1}, \mathbf{n}_{2}, \mathbf{n}_{3}$ são independentes) ficamos com

$$
\begin{aligned}
R_{0} & =\left\langle\sigma_{0}\right\rangle_{\Lambda} \sum_{\partial \mathbf{n}_{2}=\{0, g\}} \sum_{\partial \mathbf{n}_{3}=\{0, g\}} Z_{\Lambda}^{-2} \omega\left(\mathbf{n}_{2}\right) \omega\left(\mathbf{n}_{3}\right) \\
& =\left\langle\sigma_{0}\right\rangle_{\Lambda}^{3} .
\end{aligned}
$$

Lema 5.0.8.

$$
R_{u v} \leq \tanh (\beta J(v-u)) M^{2} \sum_{\substack{\partial \mathbf{n}_{2}=\{0\} \Delta\{u\} \\ \partial \mathbf{n}_{3}=\emptyset}} \frac{\omega\left(\boldsymbol{n}_{2}\right) \omega\left(\boldsymbol{n}_{3}\right)}{Z^{2}}\left\langle\sigma_{v}\right\rangle_{[C(0)]^{c} .}
$$

Demonstração. Em $R_{u v}$, para cada configuração de correntes $\mathbf{n}_{1}$, temos que o fluxo $n_{1\{u, v\}}$ é impar. Como fizemos em $T$, vamos mudar a paridade e assim ficar com a soma multiplicada pelo fator $\tanh (\beta J(v-u))$. A configuração resultante $\mathbf{n}_{1}$ tem fontes $\{0\} \Delta\{u, v, g\}$ e seu $\{0\} \Delta\{u, v, g\}$-backbone é da forma $\omega_{1} \circ \omega_{2} \operatorname{com} \omega_{1}: 0 \rightarrow u$ completamente contido em $C_{\mathbf{n}_{2}+\mathbf{n}_{3}}(g)^{c} \mathrm{e}$ $\omega_{2}: v \rightarrow g$.

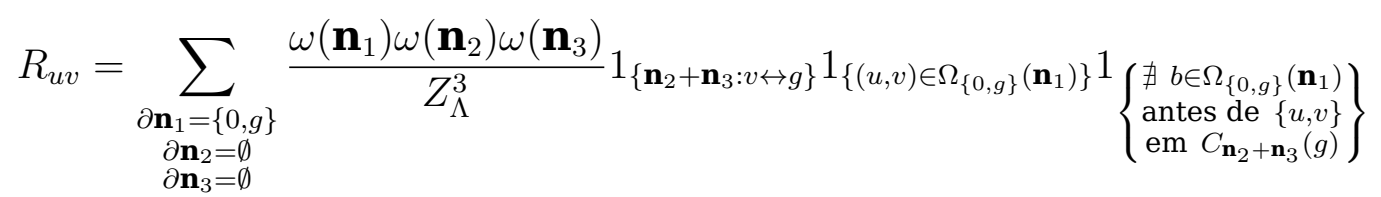

Usando a cota trivial para a função indicadora temos que

$$
R_{u v} \leq \sum_{\substack{\partial \mathbf{n}_{2}=\emptyset \\
\partial \mathbf{n}_{3}=\emptyset}} \frac{\omega\left(\mathbf{n}_{2}\right) \omega\left(\mathbf{n}_{3}\right)}{Z_{\Lambda}^{2}} 1_{\left\{\mathbf{n}_{2}+\mathbf{n}_{3}: v \leftrightarrow g\right\}} \sum_{\partial \mathbf{n}_{1}=\{0, g\}} Z_{\Lambda}^{-1} \omega\left(\mathbf{n}_{1}\right) 1_{\left\{\begin{array}{l}
\nexists b \in \Omega_{\{0, g\}}\left(\mathbf{n}_{1}\right) \\
\text { antes de }\{u, v\} \\
\operatorname{em~} \mathbf{n}_{2}+\mathbf{n}_{3}(g)
\end{array}\right\}} .
$$

Agora mudando a paridade do fluxo $n_{1\{u, v\}}$ de ímpar a par e usando a representação por correntes aleatórias, temos que o lado direito da igualdade 
acima é dado por

$$
\tanh (\beta J(u-v)) \sum_{\substack{\partial \mathbf{n}_{2}=\emptyset \\ \partial \mathbf{n}_{3}=\emptyset}} \frac{\omega\left(\mathbf{n}_{2}\right) \omega\left(\mathbf{n}_{3}\right)}{Z_{\Lambda}^{2}} 1_{\left\{\mathbf{n}_{2}+\mathbf{n}_{3}: v \leftrightarrow g\right\}} 1_{\left\{\mathbf{n}_{2}+\mathbf{n}_{3}: 0 \leftrightarrow g\right\}} \sum_{\substack{\omega_{1}: 0 \rightarrow u \\ \omega \\ \omega_{2}: v \rightarrow g}} \rho\left(\omega_{1} \circ \omega_{2}\right) 1_{\left\{\omega_{1} \cap C_{\left.\mathbf{n}_{2}+\mathbf{n}_{3}(g)=\emptyset\right\}}\right.}
$$

onde a presença do termo $1_{\left\{\mathbf{n}_{2}+\mathbf{n}_{3}: 0 \leftrightarrow g\right\}}$ se faz necessária para garantir que 0 não esteja conectado $g$ em $\mathbf{n}_{2}+\mathbf{n}_{3}$. Por outro lado,

$$
\begin{aligned}
\sum_{\substack{\omega_{1}: 0 \rightarrow u \\
\omega_{2}: v \rightarrow g}} \rho\left(\omega_{1} \circ \omega_{2}\right) 1_{\left\{\omega_{1} \cap C_{\mathbf{n}_{2}+\mathbf{n}_{3}}(g)=\emptyset\right\}} & =\sum_{\omega_{1}: 0 \rightarrow u} \rho\left(\omega_{1}\right) 1_{\left\{\omega_{1} \cap C_{\mathbf{n}_{2}+\mathbf{n}_{3}}(g)=\emptyset\right\}} \sum_{\omega_{2}: v \rightarrow g} \rho_{\omega_{1} c}\left(\omega_{2}\right) \\
& \leq \sum_{\omega_{1}: 0 \rightarrow u} \rho_{[C(g)]^{c}}\left(\omega_{1}\right)\left\langle\sigma_{v}\right\rangle_{\omega_{1}^{c}} \\
& \leq\left\langle\sigma_{v}\right\rangle\left\langle\sigma_{0} \sigma_{u}\right\rangle_{[C(g)]^{c}},
\end{aligned}
$$

onde $C(g):=C_{\mathbf{n}_{2}+\mathbf{n}_{3}}(g)$ e usamos na primeira igualdade a identidade (4.18), em seguida, (4.19) e por último (4.21) e a Desigualdade GKS-II. Substituindo a expressão acima em (5.14), obtemos

$$
R_{u v} \leq \tanh (\beta J(u-v))\left\langle\sigma_{v}\right\rangle \sum_{\substack{\partial \mathbf{n}_{2}=\emptyset \\ \partial \mathbf{n}_{3}=\emptyset}} \frac{\omega\left(\mathbf{n}_{2}\right) \omega\left(\mathbf{n}_{3}\right)}{Z_{\Lambda}^{2}}\left\langle\sigma_{0} \sigma_{u}\right\rangle_{[C(g)]^{c}} 1_{\left\{\mathbf{n}_{2}+\mathbf{n}_{3}: v \leftrightarrow g\right\}} 1_{\left\{\mathbf{n}_{2}+\mathbf{n}_{3}: 0 \leftrightarrow g\right\}} .
$$

Aplicando o Lema de Comutação podemos reescrever o lado direito como

$$
\tanh (\beta J(u-v))\left\langle\sigma_{v}\right\rangle \sum_{\substack{\partial \mathbf{n}_{2}=\{v, g\} \\ \partial \mathbf{n}_{3}=\{v, g\}}} \frac{\omega\left(\mathbf{n}_{2}\right) \omega\left(\mathbf{n}_{3}\right)}{Z_{\Lambda}^{2}}\left\langle\sigma_{0} \sigma_{u}\right\rangle_{[C(g)]^{c}} 1_{\left\{\mathbf{n}_{2}+\mathbf{n}_{3}: 0 \leftrightarrow g\right\}} .
$$

Próximo passo fazer a soma condicionando sobre o aglomerado $C_{\mathbf{n}_{2}+\mathbf{n}_{3}}(g)$ possuindo sítio fantasma, usar (4.9) juntamente com (4.14) para concluir que a expressão acima é dada por

$$
\begin{aligned}
\sum_{\substack{\partial \mathbf{n}_{2}=\{v, g\} \\
\partial \mathbf{n}_{3}=\{v, g\}}} \frac{\omega\left(\mathbf{n}_{2}\right) \omega\left(\mathbf{n}_{3}\right)}{Z_{\Lambda}^{2}}\left\langle\sigma_{0} \sigma_{u}\right\rangle_{[C(g)]^{c}} 1_{\left\{\mathbf{n}_{2}+\mathbf{n}_{3}: 0 \leftrightarrow g\right\}} & =\sum_{\substack{\partial \mathbf{n}_{2}=\{0\} \Delta\{u, v, g\} \\
\partial \mathbf{n}_{3}=\{v, g\}}} \frac{\omega\left(\mathbf{n}_{2}\right) \omega\left(\mathbf{n}_{3}\right)}{Z_{\Lambda}^{2}} 1_{\left\{\mathbf{n}_{2}+\mathbf{n}_{3}: 0 \leftrightarrow g\right\}} \\
& =\sum_{\substack{\partial \mathbf{n}_{2}=\{0\} \Delta\{u\} \\
\partial \mathbf{n}_{3}=\emptyset}} \frac{\omega\left(\mathbf{n}_{2}\right) \omega\left(\mathbf{n}_{3}\right)}{Z_{\Lambda}^{2}}\left(\left\langle\sigma_{v}\right\rangle_{[C(0)] c}\right)^{2}
\end{aligned}
$$

Na segunda igualdade de (5.16), o que fizemos foi condicionar no aglo- 
merado $C(0):=C_{\mathbf{n}_{2}+\mathbf{n}_{3}}(0)$. Agora usando (5.16) em (5.15) e também a Desigualdade GKS-II $\left(\left\langle\sigma_{v}\right\rangle_{[C(g)]^{c}} \leq\left\langle\sigma_{v}\right\rangle\right)$ segue que

$$
R_{u v} \leq \tanh (\beta J(u-v)) M^{2} \sum_{\substack{\partial \mathbf{n}_{2}=\{0\} \Delta\{u\} \\ \partial \mathbf{n}_{3}=\emptyset}} \frac{\omega\left(\mathbf{n}_{2}\right) \omega\left(\mathbf{n}_{3}\right)}{Z_{\Lambda}^{2}}\left\langle\sigma_{v}\right\rangle_{[C(0)]^{c}} .
$$

\section{Demostração do Teorema 5.0.1}

Agora que temos reunidos todos os elementos basta notar que a somatória em (5.12) é uma das parcelas de $\left\langle\sigma_{0} ; \sigma_{u} \sigma_{v}\right\rangle$, assim se somamos em $u$ e $v$, temos

$$
\sum_{u v} R_{u v} \leq \frac{M^{2}}{2} \sum_{u, v} \tanh (\beta J(u-v))\left\langle\sigma_{0} ; \sigma_{u} \sigma_{v} .\right\rangle
$$

Usando que as desigualdades (5.11), (5.8), (5.17), que $\tanh (x) \leq x$, para $x \geq 0$ juntamente com (4.12) a cota superior para (5.4) desejada, isto é,

$$
M \leq \tanh (\beta h) \chi+M^{3}+M^{2} \beta \frac{\partial M}{\partial \beta} .
$$




\section{Capítulo 6}

\section{Desigualdades Diferenciais na Classe de Griffiths-Simon}

Neste capítulo vamos mostrar a validade da desigualdade diferencial (6.14). Esta desigualdade se obtêm está num contexto mais geral que o do modelo de Ising. Agora os spins podem assumir valores reais mas desde que suas distribuições conjuntas estejam na classe de Griffiths-Simon (classe GS). Procederemos de maneira análoga a como fizemos no capítulo anterior, para obter a desigualdade principal para spins no modelo de Ising. Para assegurar a integrabilidade dos spins gerais $\varphi$ faremos a suposição permanente que ${ }^{1}$

$$
\int_{\mathbb{R}} e^{a \varphi^{2}} d \rho_{0}(\varphi)<+\infty
$$

para todo $a<\infty$. A principal referência deste capítulo é o artigo [3].

Iniciaremos considerando o spin $\varphi$ com distribuição na classe GS, escreveremos este spin como

$$
\varphi=\sum_{1 \leq \alpha \leq N} Q_{\alpha}^{(N)} \sigma_{\alpha}
$$

onde $Q_{\alpha}^{(N)}$ são coeficientes positivos e os $\sigma_{\alpha}$ são spins de Ising "microscópi$\cos$ ". Os spins $\sigma_{\alpha}$ são acoplados ferromagneticamente via o Hamiltoniano

$$
H_{1}^{(N)}=-\frac{1}{2} \sum_{\alpha, \delta} I_{\alpha, \delta}^{(N)} \sigma_{\alpha} \sigma_{\delta}
$$

Vamos usar a representação acima em cada sítio da rede. Assim, adicionamos uma segunda etiqueta $\alpha$ a cada sítio, e consideramos a matriz

${ }^{1} \rho_{0}(d \varphi), d \rho_{0}(\varphi)$ serão notações equivalentes usadas livremente 
Capítulo 6. Desigualdades Diferenciais na Classe de Griffiths-Simon 72 de sítios "microscópicos" $\left\{(x, \alpha): x \in(-L, L]^{d} ; \alpha=1, \ldots, N\right\}$ com os spins $\sigma_{(x, \alpha)}$.

Agora, cada sítio $x$ da rede será representado pelo "bloco" $B_{x}=\{(x, \alpha)$ : $\alpha=1, \ldots, N\}$, e as variáveis $\varphi_{x}$ são escritas como

$$
\varphi_{x}=\sum_{1 \leq \alpha \leq N} Q_{\alpha}^{(N)} \sigma_{(x, \alpha)} .
$$

$\triangleright$ Os sistemas com spin em GS, com interação de dois corpos macroscópicos

$$
H_{2}=-\frac{1}{2} \sum_{x, y} J(x-y) \varphi_{x} \varphi_{y}-h \sum_{x} \varphi_{x}
$$

podem ser aproximadas por modelos de Ising com Hamiltoniano total da forma

$$
\begin{aligned}
H^{(N)} & =H_{1}^{(N)}+H_{2} \\
& =-\frac{1}{2} \sum_{x, y, \alpha, \delta} J_{\{(x, \alpha)\}} \sigma_{(x, \alpha)} \sigma_{(y, \delta)}-\sum_{x, \alpha} h_{\alpha} \sigma_{(x, \alpha)},
\end{aligned}
$$

onde

$$
J_{\{(x, \alpha),(y, \delta)\}}=\left\{\begin{array}{cll}
J_{x, y} Q_{\alpha}^{(N)} Q_{\delta}^{(N)} & \text { se } & y \neq x \\
I_{x, \alpha, \delta}^{(N)} & \text { se } & y=x
\end{array}\right.
$$

$\mathrm{e}$

$$
h_{\alpha}=h Q_{\alpha}^{(N)} .
$$

Estas duas expressões anteriores foram obtidas somando e reordenando os termos de $H_{1}^{(N)}$ e $H_{2}$.

Se temos as representações por correntes aleatórias para os spin $\sigma$ então as relações podem ser expressadas em termos de funções de correlação dos "spins blocos" $\varphi_{x}$.

No capítulo anterior conseguiu-se a desigualdade principal mas para spins de Ising, agora vamos generalizá-la para spins da classe GS com o Hamiltoniano ferromagnético (6.3).

Lema 6.0.9. O modelo de Ising definido pelo Hamiltoniano (6.4) tem a seguinte representação por correntes aleatórias para $M$ :

$$
M=\sum_{\alpha} Q_{\alpha}^{(N)} \sum_{\partial n_{1}=\{(0, \alpha), g\}} \frac{w\left(n_{1}\right)}{Z}
$$


Demonstração. Como $\varphi_{x}$ pertence a GS, temos no limite termodinâmico que

$$
\begin{aligned}
M & =\left\langle\varphi_{x}\right\rangle=\left\langle\varphi_{0}\right\rangle \\
& =\lim _{\left|\Lambda^{g}\right| \nearrow \mathbb{Z}^{d}} \frac{\sum_{\omega \in \Omega_{\Lambda} g} \varphi_{0} e^{-\beta H_{\Lambda} g(\omega)}}{\sum_{\omega \in \Omega_{\Lambda} g} e^{-\beta H_{\Lambda} g(\omega)}} .
\end{aligned}
$$

Agora escrevemos $\varphi_{x}$ como (6.2) e, também, a parte do denominador sabemos que é $2^{\left|\Lambda^{g}\right|} Z$, assim que $M$ fica como

$$
\begin{aligned}
M & =\lim _{\left|\Lambda^{g}\right| \nearrow \mathbb{Z}^{d}} \frac{\sum_{\omega \in \Omega_{\Lambda} g} \sum_{\alpha} Q_{\alpha}^{(N)} \sigma_{(0, \alpha)} e^{-\beta H_{\Lambda} g(\omega)}}{2^{\left|\Lambda^{g}\right|} Z} \\
& =\lim _{\left|\Lambda^{g}\right| \nearrow \mathbb{Z}^{d}} \frac{\sum_{\alpha} \sum_{\omega \in \Omega_{\Lambda} g} Q_{\alpha}^{(N)} \sigma_{(0, \alpha)} e^{-\beta H_{\Lambda} g(\omega)}}{2^{\left|\Lambda^{g}\right|} Z} \\
& =\lim _{\left|\Lambda^{g}\right| \nearrow \mathbb{Z}^{d}} \frac{\sum_{\alpha} Q_{\alpha}^{(N)} \sum_{\omega \in \Omega_{\Lambda^{\prime}}} \sigma_{(0, \alpha)} e^{-\beta H_{\Lambda} g(\omega)}}{2^{\left|\Lambda^{g}\right|} Z} .
\end{aligned}
$$
$\operatorname{logo}$

Sabemos do capítulo 4 que $\sum_{\omega \in \Omega_{\Lambda} g} \sigma_{(0, \alpha)} e^{-\beta H_{\Lambda} g(\omega)}=2^{\left|\Lambda^{g}\right|} \sum_{\mathbf{n}_{1}} w\left(\mathbf{n}_{1}\right) 1_{\partial \mathbf{n}_{1}=\{(0, \alpha), g\}}$,

$$
M=\sum_{\alpha} Q_{\alpha}^{(N)} \sum_{\mathbf{n}_{1}=\{(0, \alpha), g\}} \frac{w\left(\mathbf{n}_{1}\right)}{Z} .
$$

Com o objetivo de deduzir a desigualdade diferencial (6.14), usaremos a mesma técnica empregada no Capítulo 4, ou seja, vamos adicionar à igualdade (6.7) duas somas "falsas"e em seguida, fazer a decomposição apropriada. Para esta decomposição, precisaremos da versão para blocos do Lema da Comutação (switching lemma). Isto motiva a seguinte definição.

n conecta B. Dada uma coleção de sítios $B$ e uma configuração de correntes $\mathbf{n}$, nós diremos que $\mathbf{n}$ conecta $B$ com outro sitio $z$, e denotamos esta condição por $\mathbf{n}: B \leftrightarrow z$, se existe um $x \in B$ tal que $\mathbf{n}: x \leftrightarrow z$. 
Capítulo 6. Desigualdades Diferenciais na Classe de Griffiths-Simon 74

Lema 6.0.10. Sejam B uma coleção de sítios e z um sítio fora de B. Então, para qualquer função $f$ definida sobre configurações de correntes que é decrescente em cada numero de fluxo, temos

$$
\begin{aligned}
\sum_{\substack{\partial \boldsymbol{n}_{1}=\emptyset \\
\partial \mathbf{n}_{2}=\emptyset}} w\left(\boldsymbol{n}_{1}\right) w\left(\boldsymbol{n}_{2}\right) f\left(\boldsymbol{n}_{1}+\right. & \left.\boldsymbol{n}_{2}\right) 1_{\left\{\boldsymbol{n}_{1}+\boldsymbol{n}_{2}: B \leftrightarrow z\right\}} \\
& \leq \sum_{\substack{x \in B \\
y \in B^{c}}} \beta J(y-x) \sum_{\substack{\partial \boldsymbol{n}_{1}=\{x, y\} \\
\partial \mathbf{n}_{2}=\{x\} \Delta\{y\}}} w\left(\boldsymbol{n}_{1}\right) w\left(\boldsymbol{n}_{2}\right) f\left(\boldsymbol{n}_{1}+\boldsymbol{n}_{2}\right)
\end{aligned}
$$

Demonstração. Já que $\mathbf{n}_{1}+\mathbf{n}_{2}$ não tem fontes, então o fluxo total das arestas que conectam $B$ com $B^{c}$ deve ser ao menos 2, logo

$$
1_{\left\{\mathbf{n}_{1}+\mathbf{n}_{2}: B \leftrightarrow z\right\}} \leq \sum_{\substack{x \in B \\ y \in B^{c}}} \frac{n_{1\{x, y\}}+n_{2\{x, y\}}}{2} 1_{\left\{\mathbf{n}_{1}+\left.\mathbf{n}_{2}\right|_{B^{c}}: y \leftrightarrow z\right\}} .
$$

Esta desigualdade (6.9) nos permite limitar o lado esquerdo (LE) de (6.8):

$$
L E \leq \frac{1}{2} \sum_{\substack{x \in B^{c} \\ y \in B^{c}}} \sum_{\mathbf{n}_{1}=\emptyset}\left\{n_{1\{x, y\}} w\left(\mathbf{n}_{1}\right) w\left(\mathbf{n}_{2}\right) f\left(\mathbf{n}_{1}+\mathbf{n}_{2}\right) 1_{\left\{\mathbf{n}_{1}+\left.\mathbf{n}_{2}\right|_{B} c: y \leftrightarrow z\right\}}+\left(\mathbf{n}_{1} \Leftrightarrow \mathbf{n}_{2}\right)\right\},
$$

onde o simbolo $\left(\mathbf{n}_{1} \Leftrightarrow \mathbf{n}_{2}\right.$ ) significa que trocamos $\mathbf{n}_{1}$ por $\mathbf{n}_{2}$ na expressão antes do + , Por outro lado, segue de (4.3) que

$$
\begin{aligned}
n_{1\{x, y\}} w\left(\mathbf{n}_{1}\right) & =\frac{(\beta J(y-x))^{n_{1\{x, y\}}}}{\left(n_{1\{x, y\}}-1\right) !} \prod_{b \neq\{x, y\}} \frac{\left(\beta J_{b}\right)^{n_{b}}}{n_{b} !} \\
& =\beta J(y-x) w\left(\mathbf{n}_{1}-\delta_{b,\{x, y\}}\right),
\end{aligned}
$$

onde a notação $\mathbf{n}_{1}-\delta_{b,\{x, y\}}$ significa que na configuração de corrente $\mathbf{n}_{1}$ o fluxo correspondente a aresta $\{x, y\}$ é reduzido em uma unidade, assim seu peso é dado por

$$
w\left(\mathbf{n}_{1}-\delta_{b,\{x, y\}}\right)=\frac{(\beta J(y-x))^{n_{1\{x, y\}}-1}}{\left(n_{1\{x, y\}}-1\right) !} \prod_{b \neq\{x, y\}} \frac{\left(\beta J_{b}\right)^{n_{b}}}{n_{b} !} .
$$

Agora, substituindo esta expressão em (6.9):

$$
L E \leq \sum_{\substack{x \in B \\ y \in B^{c}}} \beta J(y-x) \sum_{\substack{\partial \mathbf{n}_{1}=\{x, y\} \\ \partial \mathbf{n}_{2}=\emptyset}} w\left(\mathbf{n}_{1}\right) w\left(\mathbf{n}_{2}\right) f\left(\mathbf{n}_{1}+\mathbf{n}_{2}+\delta_{b,\{x, y\}}\right) 1_{\left\{\mathbf{n}_{1}+\mathbf{n}_{2}+\left.\delta_{b,\{x, y\}}\right|_{B c}: y \leftrightarrow z\right\}} .
$$


Aqui aumentamos em uma unidade o fluxo da aresta $\{x, y\}$ na configuração $\mathbf{n}_{1}$. Isto faz que surjam duas fontes $x$ e $y$, ou seja, $\partial \mathbf{n}_{1}=\{x, y\}$.

Agora, vamos limitar $f\left(\mathbf{n}_{1}+\mathbf{n}_{2}+\delta_{b,\{x, y\}}\right)$. Para fazer isso vamos usar a hipótese de monotonicidade de $f, f\left(\mathbf{n}_{1}+\mathbf{n}_{2}+\delta_{b,\{x, y\}}\right) \leq f\left(\mathbf{n}_{1}+\mathbf{n}_{2}\right)$, e também uma cota superior para $1_{\left\{\mathbf{n}_{1}+\mathbf{n}_{2}+\left.\delta_{b,\{x, y\}}\right|_{B}: y \leftrightarrow z\right\}}$ que é mostrada a seguir. Se $\{x, y\} \notin \mathbf{B}^{c}$, onde $\mathbf{B}$ é o conjunto de arestas com ao menos um ponto final em $B$, então

$$
\begin{aligned}
1_{\left\{\left(\mathbf{n}_{1}+\mathbf{n}_{2}+\delta_{b,\{x, y\}}||_{B} c: y \leftrightarrow z\right\}\right.} & =1_{\left\{\left.\left(\mathbf{n}_{1}+\mathbf{n}_{2}\right)\right|_{B^{c}}: y \leftrightarrow z\right\}} \\
& \leq 1_{\left\{\mathbf{n}_{1}+\mathbf{n}_{2}: y \leftrightarrow z\right\}} .
\end{aligned}
$$

Combinando estas duas estimativas se obtém de (6.12) que

$$
L E \leq \sum_{\substack{x \in B \\ y \in B^{c}}} \beta J(y-x) \sum_{\substack{\partial \mathbf{n}_{1}=\{x, y\} \\ \partial \mathbf{n}_{2}=\emptyset}} w\left(\mathbf{n}_{1}\right) w\left(\mathbf{n}_{2}\right) f\left(\mathbf{n}_{1}+\mathbf{n}_{2}\right) 1_{\left\{\mathbf{n}_{1}+\mathbf{n}_{2}: y \leftrightarrow z\right\}}
$$

Agora podemos usar o Lema da Comutação visto no Capítulo $5 \operatorname{com} g=z$, e deduzir que

$$
L E \leq \sum_{\substack{x \in B \\ y \in B^{c}}} \beta J(y-x) \sum_{\substack{\partial \mathbf{n}_{1}=\{x, y\} \\ \partial \mathbf{n}_{2}=\{x\} \Delta\{y\}}} w\left(\mathbf{n}_{1}\right) w\left(\mathbf{n}_{2}\right) f\left(\mathbf{n}_{1}+\mathbf{n}_{2}\right) .
$$

Como consequência do fato provado acima temos o seguinte corolário

Corolário 6.0.1. Se f é uma função decrescente de números de fluxo, então

$$
\begin{gathered}
\sum_{\substack{\partial \mathbf{n}_{1}=\emptyset \\
\partial \mathbf{n}_{2}=\emptyset}} w\left(\boldsymbol{n}_{1}\right) w\left(\boldsymbol{n}_{2}\right) f\left(\boldsymbol{n}_{1}+\boldsymbol{n}_{2}\right) 1_{\left\{\boldsymbol{n}_{1}+\boldsymbol{n}_{2}: B_{v} \leftrightarrow g\right\}} \leq \sum_{\alpha} \beta h_{\alpha} \sum_{\substack{\partial \mathbf{n}_{1}=\{(v, \alpha), g\} \\
\partial \mathbf{n}_{2}=\emptyset}} w\left(\boldsymbol{n}_{1}\right) w\left(\boldsymbol{n}_{2}\right) f\left(\boldsymbol{n}_{1}+\boldsymbol{n}_{2}\right) \\
+\sum_{\alpha, y \neq v, \delta} \beta J_{\{(v, \alpha),(y, \delta)\}} \sum_{\substack{\partial \mathbf{n}_{1}=\{(v, \alpha), g\} \\
\partial \mathbf{n}_{2}=\{(y, \delta), g\}}} w\left(\boldsymbol{n}_{1}\right) w\left(\boldsymbol{n}_{2}\right) f\left(\boldsymbol{n}_{1}+\boldsymbol{n}_{2}\right)
\end{gathered}
$$

$\mathrm{Na}$ apresentação deste texto falamos que o teorema 7.0.3 usa na sua demonstração os Teoremas 6.0.2 e 5.0.1. Agora vamos mostrar o teorema 6.0 .2 pode ser obtida a partir do teorema 5.0.1 usando a versão de "blocos" dos métodos usados no Capítulo 5 . 
Capítulo 6. Desigualdades Diferenciais na Classe de Griffiths-Simon 76

Teorema 6.0.2 (Teorema 2 (ii)). Para spins na classe GS com o Hamiltoniano (6.3), temos a seguinte desigualdade

$$
M \leq \beta h \chi+\left(\beta h M^{2}+\beta|J| M^{3}\right)+\left(\beta h M+\beta|J|+M^{2}\right) \beta \frac{\partial M}{\partial \beta} .
$$

Demonstração. Começamos com

$$
M=\sum_{\alpha} Q_{\alpha} \sum_{\substack{\partial \mathbf{n}_{1}=\{(0, \alpha), g\} \\ \partial \mathbf{n}_{2}=\emptyset \\ \partial \mathbf{n}_{2}=\emptyset}} Z_{\Lambda}^{-3} w\left(\mathbf{n}_{1}\right) w\left(\mathbf{n}_{2}\right) w\left(\mathbf{n}_{3}\right)
$$

que é (6.7) com duas "somas falsas". Vamos decompor (6.15) como no Capitulo (5), exceto pela condição, que um sítio que conecta a g é substituído por uma condição de bloco, e como no Capítulo (5) distinguimos três casos:

Caso 1. Não há tal bloco. Afirmação

$$
T \leq \beta h \chi .
$$

\section{Prova da Afirmação.}

$$
\begin{aligned}
& T=\sum_{x, \delta} \tanh \left(\beta h_{\delta}\right) \times
\end{aligned}
$$

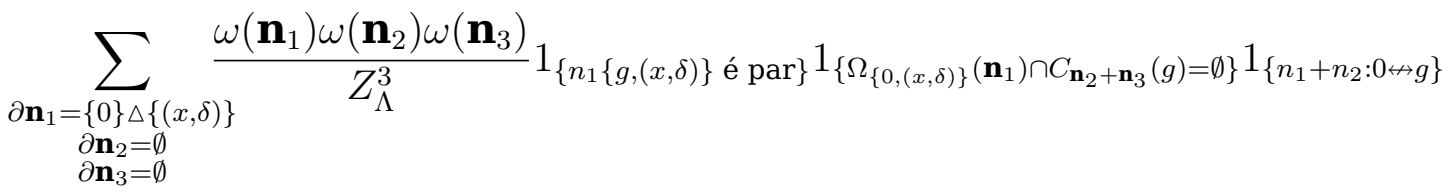

como a função indicadora é limitada por 1, temos

$$
\left.T \leq \sum_{x, \delta} \tanh \left(\beta h_{\delta}\right) \sum_{\substack{\partial \mathbf{n}_{1}=\{0\} \Delta\{(x, \delta)\} \\ \partial \mathbf{n}_{2}=\emptyset \\ \partial \mathbf{n}_{3}=\emptyset}} \frac{\omega\left(\mathbf{n}_{1}\right) \omega\left(\mathbf{n}_{2}\right) \omega\left(\mathbf{n}_{3}\right)}{Z_{\Lambda}^{3}} 1_{\left\{\Omega_{\{0,(x, \delta)\}}\left(\mathbf{n}_{1}\right) \cap C_{\left.\mathbf{n}_{2}+\mathbf{n}_{3}(g)=\emptyset\right\}}\right.} 1_{\left\{n_{1}+n_{2}: 0 \leftrightarrow g\right\}}\right\}
$$

rearranjando a ordem das somas ficamos com

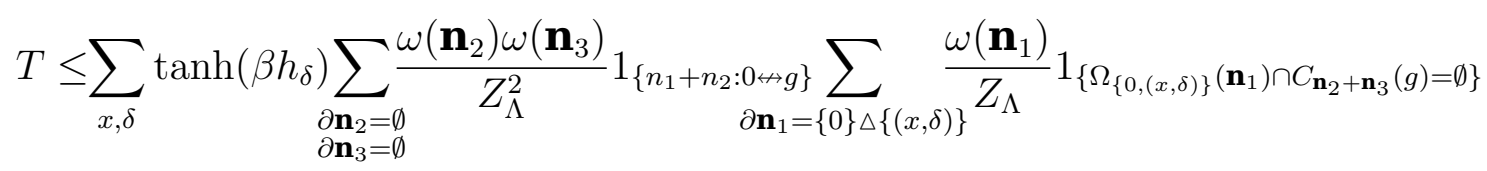


considerando o sitio 0 como um bloco, temos que

$$
\begin{aligned}
T \leq & \sum_{x, \delta} \tanh \left(\beta h_{\delta}\right) \sum_{\substack{\partial \mathbf{n}_{2}=\emptyset \\
\partial \mathbf{n}_{3}=\emptyset}} \frac{\omega\left(\mathbf{n}_{2}\right) \omega\left(\mathbf{n}_{3}\right)}{Z_{\Lambda}^{-2}} \times \\
& \left.\sum_{\alpha} Q_{\alpha} \sum_{\partial \mathbf{n}_{1}=\{(0, \alpha)\} \Delta\{(x, \delta)\}} Z_{\Lambda}^{-1} \omega\left(\mathbf{n}_{1}\right) 1_{\left\{\Omega_{\{(0, \alpha),(x, \delta)\}}\left(\mathbf{n}_{1}\right) \cap C_{\left.\mathbf{n}_{2}+\mathbf{n}_{3}(g)=\emptyset\right\}}\right.} 1_{\left\{n_{1}+n_{2}:(0, \alpha) \leftrightarrow g\right\}}\right\},
\end{aligned}
$$

e usando (4.20), ou seja, a representação por caminhos aleatórios, mas sem seguir por caminhos de $C_{\mathbf{n}_{2}+\mathbf{n}_{3}}$, obtemos

$$
T \leq \sum_{\alpha} Q_{\alpha} \sum_{x, \delta} \tanh \left(\beta h_{\delta}\right) \sum_{\substack{\partial \mathbf{n}_{2}=\emptyset \\ \partial \mathbf{n}_{3}=\emptyset}} \frac{\omega\left(\mathbf{n}_{2}\right) \omega\left(\mathbf{n}_{3}\right)}{Z_{\Lambda}^{2}} 1_{\left\{n_{1}+n_{2}:(0, \alpha) \leftrightarrow g\right\}} \sum_{\omega:(0, \alpha) \rightarrow(x, \delta)} \rho_{[C(g)]^{c}} 1_{\left\{\omega \cap C_{\left.\mathbf{n}_{2}+\mathbf{n}_{3}(g)=\emptyset\right\}}\right.}
$$

Fazendo uso de (4.21) e reordenando as somas temos

$$
\left.T \leq \sum_{\alpha} Q_{\alpha} \sum_{x, \delta} \tanh \left(\beta h_{\delta}\right) \sum_{\substack{\partial \mathbf{n}_{2}=\emptyset \\ \partial \mathbf{n}_{3}=\emptyset}} \frac{\omega\left(\mathbf{n}_{2}\right) \omega\left(\mathbf{n}_{3}\right)}{Z_{\Lambda}^{2}} 1_{\left\{n_{1}+n_{2}:(0, \alpha) \leftrightarrow g\right\}}\left\langle\sigma_{(0, \alpha)} \sigma_{(x, \delta)}\right\rangle_{[C(g)]^{c}}\right\} .
$$

Já que $\tanh (x) \leq x$ para todo $x \geq 0, h_{\delta}=h Q_{\delta}$ segue de (4.14) que

$$
T \leq \beta h \sum_{\alpha, x, \delta} Q_{\alpha} Q_{\delta}\left\langle\sigma_{(0, \alpha)} ; \sigma_{(x, \delta)}\right\rangle=\beta h \chi
$$

Caso 2. O primeiro bloco contém a origem. Para este caso vamos mostrar que usando (6.13) em $\mathbf{n}_{2}$ e $\mathbf{n}_{3}$ e também (6.7) que temos a seguinte desigualdade

$$
R_{0} \leq \beta h M^{2}+\beta\|J\| M^{3}
$$

Para provar a afirmação primeiro observamos que

$$
R_{0}=\sum_{\substack{\partial \mathbf{n}_{1}=\{0, g\} \\ \partial \mathbf{n}_{2}=\emptyset \\ \partial \mathbf{n}_{3}=\emptyset}} \frac{\omega\left(\mathbf{n}_{1}\right) \omega\left(\mathbf{n}_{2}\right) \omega\left(\mathbf{n}_{3}\right)}{Z_{\Lambda}^{3}} 1_{\left\{\mathbf{n}_{2}+\mathbf{n}_{3}: 0 \leftrightarrow g\right\}},
$$

considerando o sítio 0 como um bloco, seria o bloco $\{(0, \alpha)\}$, que chamare- 
Capítulo 6. Desigualdades Diferenciais na Classe de Griffiths-Simon 78 mos de $B_{0}$, temos

$$
\begin{aligned}
R_{0} & =\sum_{\alpha} Q_{\alpha} \sum_{\substack{\partial \mathbf{n}_{1}=\{(0, \alpha), g\} \\
\partial \mathbf{n}_{2}=\emptyset \\
\partial \mathbf{n}_{3}=\emptyset}} \omega\left(\mathbf{n}_{1}\right) \omega\left(\mathbf{n}_{2}\right) \omega\left(\mathbf{n}_{3}\right) 1_{\left\{\mathbf{n}_{2}+\mathbf{n}_{3}:(0, \alpha) \leftrightarrow g\right\}} \\
& =\sum_{\alpha} Q_{\alpha} \sum_{\substack{\hat{n}_{1}=\{(0, \alpha), g\} \\
\partial \mathbf{n}_{2}=\emptyset \\
\partial \mathbf{n}_{3}=\emptyset}} \omega\left(\mathbf{n}_{1}\right) \omega\left(\mathbf{n}_{2}\right) \omega\left(\mathbf{n}_{3}\right) 1_{\left\{\mathbf{n}_{2}+\mathbf{n}_{3}: B_{0} \leftrightarrow g\right\}},
\end{aligned}
$$

e, ordenando os termos, temos

$$
R_{0}=\sum_{\alpha} Q_{\alpha} \sum_{\partial \mathbf{n}_{1}=\{(0, \alpha), g\}} \frac{\omega\left(\mathbf{n}_{1}\right)}{Z_{\Lambda}} \sum_{\substack{\partial \mathbf{n}_{2}=\emptyset \\ \partial \mathbf{n}_{3}=\emptyset}} \frac{\omega\left(\mathbf{n}_{2}\right) \omega\left(\mathbf{n}_{3}\right)}{Z_{\Lambda}^{2}} 1_{\left\{\mathbf{n}_{2}+\mathbf{n}_{3}: B_{0} \leftrightarrow g\right\}} .
$$

Agora usando (6.13) nas configurações $\mathbf{n}_{2}$ e $\mathbf{n}_{3}$, obtemos

$$
\begin{aligned}
R_{0} \leq \sum_{\alpha} Q_{\alpha} \sum_{\partial \mathbf{n}_{1}=\{(0, \alpha), g\}} \frac{\omega\left(\mathbf{n}_{1}\right)}{Z_{\Lambda}}\left[\sum_{\alpha} \beta h_{\alpha} \sum_{\substack{\partial \mathbf{n}_{2}=\{(0, \alpha), g\} \\
\partial \mathbf{n}_{3}=\emptyset}} \frac{\omega\left(\mathbf{n}_{2}\right) \omega\left(\mathbf{n}_{3}\right)}{Z_{\Lambda}}\right. & \\
& \left.+\sum_{\alpha, y \neq 0, \delta} \beta J_{\{(0, \alpha),(y, \delta)\}} \sum_{\substack{\partial \mathbf{n}_{2}=\{(0, \alpha), g\} \\
\partial \mathbf{n}_{3}=\{(y, \delta), g\}}} \frac{\omega\left(\mathbf{n}_{2}\right) \omega\left(\mathbf{n}_{3}\right)}{Z_{\Lambda}^{2}}\right] .
\end{aligned}
$$

Usando o fato que $h_{\alpha}=h Q_{\alpha}$ e reordenando as somas de forma apropriada ficamos com a seguinte estimativa:

$$
\begin{aligned}
R_{0} \leq \beta h \sum_{\alpha} Q_{\alpha} \sum_{\partial \mathbf{n}_{1}=\{(0, \alpha), g\}} \frac{\omega\left(\mathbf{n}_{1}\right)}{Z_{\Lambda}} \sum_{\alpha} Q_{\alpha} \sum_{\substack{\partial \mathbf{n}_{2}=\{(0, \alpha), g\} \\
\partial \mathbf{n}_{3}=\emptyset}} \frac{\omega\left(\mathbf{n}_{2}\right) \omega\left(\mathbf{n}_{3}\right)}{Z_{\Lambda}^{2}} \\
+\sum_{\alpha} Q_{\alpha} \sum_{\partial \mathbf{n}_{1}=\{(0, \alpha), g\}} \frac{\omega\left(\mathbf{n}_{1}\right)}{Z_{\Lambda}} \sum_{\alpha, y \neq 0, \delta} \beta J_{\{(0, \alpha),(y, \delta)\}} \sum_{\substack{\partial \mathbf{n}_{2}=\{(0, \alpha), g\} \\
\partial \mathbf{n}_{3}=\{(y, \delta), g\}}} \frac{\omega\left(\mathbf{n}_{2}\right) \omega\left(\mathbf{n}_{3}\right)}{Z_{\Lambda}^{2}} .
\end{aligned}
$$

Agora vamos usar (6.7) juntamente com $J_{\{(0, \alpha),(y, \delta)\}}=J_{0, y} Q_{\alpha} Q_{\delta}$, quando $0 \neq y$ para concluir que

$$
R_{0} \leq\left\langle\varphi_{0}\right\rangle^{2}+\sum_{\alpha, y \neq 0, \delta} \beta J_{0, y}\left\langle\varphi_{0}\right\rangle^{2}\left\langle\varphi_{y}\right\rangle .
$$

Tomando o Limite Termodinâmico nas funções de correlação acima e usando a invariância translacional do modelo com condição de contorno livre, te- 
mos que

$$
\begin{aligned}
R_{0} & \leq M^{2}+\sum_{\alpha, y \neq 0, \delta} \beta J_{0, y} M^{3} \\
& \leq M^{2}+\beta\|J\| M^{3} .
\end{aligned}
$$

3. O primeiro bloco $B_{v}$ corresponde a $v \neq 0$. Neste caso temos o seguinte resultado:

$$
\sum_{\alpha, u, \delta, v, \gamma} R_{\{\alpha,(u, v),(v, \gamma)\}} \leq\left(\beta h M+\beta|J| M^{2}\right) \beta \frac{\partial M}{\partial \beta} .
$$

Para provar este fato vamos começar observando que

$$
\sum_{\alpha, u, \delta, v, \gamma} R_{\{\alpha,(u, v),(v, \gamma)\}}
$$

representa a contribuição das configurações onde $\{\alpha,(u, \delta),(v, \gamma)\}$ indica que $((u, \delta),(v, \gamma))$ é o primeiro passo do backbone $\Omega_{\{(0, \alpha), g\}}\left(\mathbf{n}_{1}\right)$ com um vértice final em $B_{v}$.

Da mesma forma que procedemos no modelo de Ising nós modificamos $n_{1\{(u, \delta),(v, \gamma)\}}$ de ímpar para par. Assim, teremos um fator $\tanh \left(\beta J_{\{(u, \delta),(v, \gamma)\}}\right)$ e o backbone $\Omega_{\{(0, \alpha), g\}}\left(\mathbf{n}_{1}\right)$ é composto por dois caminhos $\omega_{1}:(0, \alpha) \rightarrow(u, \delta)$, que está contido em $C_{\mathbf{n}_{2}+\mathbf{n}_{3}}(g)^{c}$, e $\omega_{1}:(v, \gamma) \rightarrow g$.

Procedendo de forma similar à feita na prova de (5.12), só que agora generalizado para blocos, obtemos

$$
\begin{aligned}
& R_{\{\alpha,(u, v),(v, \gamma)\}} \leq Q_{\alpha} \tanh \left(\beta J_{\{(u, v),(v, \gamma)\}}\right) \times \\
& \quad \times \sum_{\substack{\partial \mathbf{n}_{2}=\emptyset \\
\partial \mathbf{n}_{3}=\emptyset}} \frac{\omega\left(\mathbf{n}_{2}\right) \omega\left(\mathbf{n}_{3}\right)}{Z_{\Lambda}^{2}} 1_{\left\{\mathbf{n}_{2}+\mathbf{n}_{3}:(0, \alpha) \leftrightarrow g\right\}} 1_{\left\{\mathbf{n}_{2}+\mathbf{n}_{3}: B_{v} \leftrightarrow g\right\}} \sum_{\begin{array}{c}
\omega_{1}:(0, \alpha) \rightarrow(u, \delta) \\
\omega_{2}:(v, \gamma) \rightarrow g
\end{array}} \rho\left(\omega_{1} \circ \omega_{2}\right) 1_{\left\{\omega_{1} \cap C_{\mathbf{n}_{2}+\mathbf{n}_{3}}(g)=\emptyset\right\}} .
\end{aligned}
$$

Vamos trabalhar no somatório, envolvendo caminhos aleatórios. Primeiro observamos que (4.15) fornece a seguinte igualdade

$$
\sum_{\substack{\omega_{1}:(0, \alpha) \rightarrow(u, \delta) \\ \omega_{2}:(v, \gamma) \rightarrow g}} \rho\left(\omega_{1} \circ \omega_{2}\right) 1_{\left.\left\{\omega_{1} \cap C_{\mathbf{n}_{2}+\mathbf{n}_{3}}(g)=\emptyset\right\}\right\}}=\sum_{\substack{\omega_{1}:(0, \alpha) \rightarrow(u, \delta) \\ \omega_{2}:(v, \gamma) \rightarrow g}} \rho\left(\omega_{1}\right) \rho_{\omega_{1} c}\left(\omega_{2}\right) 1_{\left.\left\{\omega_{1} \cap C_{\mathbf{n}_{2}+\mathbf{n}_{3}}(g)=\emptyset\right\}\right\}}
$$


Capítulo 6. Desigualdades Diferenciais na Classe de Griffiths-Simon 80

Rearranjando a ordem das somas e usando (4.18) podemos ver que o lado direito da igualdade é dado por

$$
\sum_{\omega_{1}:(0, \alpha) \rightarrow(u, \delta)} \rho\left(\omega_{1}\right) 1_{\left.\left\{\omega_{1} \cap C_{\mathbf{n}_{2}+\mathbf{n}_{3}}(g)=\emptyset\right\}\right\}} \sum_{\omega_{2}:(v, \gamma) \rightarrow g} \rho_{\omega_{1}^{c}}\left(\omega_{2}\right) .
$$

De GKS-II e (4.21) segue que podemos cotar esta expressão por

$$
\sum_{\omega_{1}:(0, \alpha) \rightarrow(u, \delta)} \rho_{[C(g)]^{c}}\left(\omega_{1}\right)\left\langle\sigma_{(v, \gamma)}\right\rangle_{\omega_{1}^{c}} \leq\left\langle\sigma_{(v, \gamma)}\right\rangle\left\langle\sigma_{(0, \alpha)} \sigma_{(u, \delta)}\right\rangle_{[C(g)]^{c}} .
$$

Agora, usando esta estimativa em (6.19), temos que

$$
\begin{aligned}
R_{\{\alpha,(u, v),(v, \gamma)\}} \leq & Q_{\alpha} \tanh \left(\beta J_{\{(u, v),(v, \gamma)\}}\right) \times \\
& \sum_{\substack{\partial \mathbf{n}_{2}=\emptyset \\
\partial \mathbf{n}_{3}=\emptyset}} \frac{\omega\left(\mathbf{n}_{2}\right) \omega\left(\mathbf{n}_{3}\right)}{Z_{\Lambda}^{2}} 1_{\left\{\mathbf{n}_{2}+\mathbf{n}_{3}:(0, \alpha) \leftrightarrow g\right\}} 1_{\left\{\mathbf{n}_{2}+\mathbf{n}_{3}: B v \leftrightarrow g\right\}}\left\langle\sigma_{(v, \gamma)}\right\rangle\left\langle\sigma_{(0, \alpha)} \sigma_{(u, \delta)}\right\rangle_{[C(g)]^{c}}
\end{aligned}
$$

Considerando $f\left(\mathbf{n}_{2}+\mathbf{n}_{3}\right)=\left\langle\sigma_{(0, \alpha)} \sigma_{(u, \delta)}\right\rangle_{[C(g)]^{c}} 1_{\left\{\mathbf{n}_{2}+\mathbf{n}_{3}:(0, \alpha) \leftrightarrow g\right\}}$ e notando que ela é decrescente, segue do corolário (6.13) que a expressão acima é cotada por

$$
\begin{aligned}
& Q_{\alpha} \tanh \left(\beta J_{\{(u, v),(v, \gamma)\}}\right)\left\langle\sigma_{(v, \gamma)}\right\rangle Z_{\Lambda}^{-2} \sum_{\eta} \beta h_{\eta} \\
& \sum_{\substack{\partial \mathbf{n}_{2}=\{(v, \eta), g\} \\
\partial \mathbf{n}_{3}=\emptyset}} \omega\left(\mathbf{n}_{2}\right) \omega\left(\mathbf{n}_{3}\right) f\left(\mathbf{n}_{2}+\mathbf{n}_{3}\right)+\sum_{\eta, y \neq v, \xi} \beta J_{\{(v, \eta),(y, \xi)\}} \sum_{\substack{\partial \mathbf{n}_{2}=\{(v, \eta), g\} \\
\partial \mathbf{n}_{3}=\{(y, \xi), g\}}} \omega\left(\mathbf{n}_{2}\right) \omega\left(\mathbf{n}_{3}\right) f\left(\mathbf{n}_{2}+\mathbf{n}_{3}\right)
\end{aligned}
$$

Aplicando 6.22 e somando sobre todos $\alpha, u, \delta, v, \gamma$, obtemos,

$$
\sum_{\alpha, u, \delta, v, \gamma} R_{\{\alpha,(u, v),(v, \gamma)\}} \leq \frac{1}{2} \beta \sum_{u, v} J(v-u)\left\langle\varphi_{v}\right\rangle\left(\beta h+\beta \sum_{y} J(v-y)\left\langle\varphi_{y}\right\rangle\right)\left\langle\varphi ; \varphi_{u} \varphi_{v}\right\rangle .
$$

O fator $1 / 2$ acima vem de (4.13). Relembrando (4.12), temos

$$
\sum_{\alpha, u, \delta, v, \gamma} R_{\{\alpha,(u, v),(v, \gamma)\}} \leq\left(\beta h M+\beta|J| M^{2}\right) \beta \frac{\partial M}{\partial \beta} .
$$

Por último vemos que para terminar a prova do Teorema 6.14 item (ii), basta somar as desigualdades (6.16), (6.17) e (6.18). 


\section{Parte III}

\section{Igualdade das Temperaturas Críticas $\beta_{c}=\beta_{m}$}





\section{Capítulo 7}

\section{Consequências das Desigualdades Diferenciais}

O objetivo principal deste capítulo é provar o Teorema 7.0.3, que fala entre outras coisas, que o ponto crítico é único para magnetização e susceptibilidade. Para isso vamos fazer uso das desigualdades principais já deduzidas nos dois capítulos anteriores. Faremos uso também de algumas desigualdades conhecidas para o modelo de Percolação de Bernoulli, que estão em [2]. Entre as referências usadas estão, a já mencionadas anteriormente, [15] e principalmente [3].

Observação 7.0.3. Nesta seção vamos usar livremente as notações $M$ ou $M(\beta, h)$ para denotar a magnetização.

As desigualdades diferencias parciais que serão usadas são: A desigualdade principal (5.1) dada pelo Teorema 5.0.1

$$
M \leq \tanh (\beta h) \frac{\partial M}{\partial(\beta h)}+M^{2}\left(\beta \frac{\partial M}{\partial \beta}+M\right)
$$

a desigualdade (6.14) fornecida pelo Teorema (6.0.2)

$$
M \leq \beta h \frac{\partial M}{\partial(\beta h)}+\left(\beta|J| M^{2}+\beta h M\right)\left(\beta \frac{\partial M}{\partial \beta}+M\right)
$$

e por último as desigualdade de Aizenman-Barsky [2], dadas por

$$
\frac{\partial M}{\partial(\beta)} \leq \beta|J| M \frac{\partial M}{\partial(\beta h)}
$$


e

$$
\frac{\partial M}{\partial(\beta h)} \leq \frac{M}{\beta h}
$$

Teorema 7.0.3 (Teorema 1). Para o modelo de Ising, ou para um modelo geral na classe de Griffiths-Simon, com o Hamiltoniano (4.1) em $\mathbf{Z}^{d}(d \geq 1)$, existe uma temperatura crítica $\beta_{c} \in[0, \infty]$ com as seguintes propriedades:

(i) Para todo $0 \leq \beta<\beta_{c}$ há um único estado de Gibbs a volume infinito, a magnetização espontânea se anula, i.e. $\lim _{h \rightarrow 0^{+}} M(\beta, h) \equiv M_{+}(\beta)=0$, também temos que a susceptibilidade magnética é finita

$$
\chi(\beta, 0)=\sum_{x \in \mathbb{Z}^{d}}\left\langle\sigma_{0} \sigma_{x}\right\rangle_{\beta, h=0}<\infty .
$$

(ii) Para todo $\beta>\beta_{c}$ há quebra de simetria, com

$$
M_{+}(\beta) \geq \operatorname{const}\left[\frac{\left(\beta-\beta_{c}\right)}{\beta_{c}}\right]^{\frac{1}{2}} .
$$

(iii) $\operatorname{Em} \beta=\beta_{c}$, a magnetização em um campo magnético positivo $h \rightarrow 0^{+}$ não decresce mais rápido que o seguinte limite:

$$
M\left(\beta_{c}, h\right) \equiv\left\langle\sigma_{0}\right\rangle_{\beta_{c}, h} \geq \text { const } h^{\frac{1}{3}} .
$$

Observação 7.0.4. As desigualdades (7.3) e (7.4) determinam cotas para os chamados expoentes críticos dados por

$$
\hat{\beta}:=\limsup _{\beta \rightarrow \beta_{c}^{+}} \frac{\log M_{+}(\beta)}{\log \left(\beta-\beta_{c}\right)}
$$

$e$

$$
\delta:=\liminf _{h \rightarrow 0^{+}} \frac{\log h}{\log M\left(\beta_{c}, h\right)} .
$$

Assumindo (7.3) e (7.4) temos que

$$
\delta \geq 3 \quad \text { e } \quad \hat{\beta} \leq \frac{1}{2} .
$$

Observação 7.0.5. Do artigo [4] temos quando $\beta=\beta_{c}$, em modelos de Ising, a magnetização espontânea desaparece $\left(M_{+}\left(\beta_{c}\right)=0\right)$, ou seja, não há transição de fase em $\beta_{c}$. Isto é uma extensão da parte (i) do Teorema 1. 


\subsection{Prova dos Principais Resultados}

As duas desigualdades diferenciais parciais (5.1) e (6.14) nas duas variáveis $\beta$ e $\beta h$ serão fundamentais no estudo de três diferentes regimes do modelo (regime de altas temperaturas, regime de baixas temperaturas e na temperatura crítica).

Os pontos críticos $\beta_{c}$ e $\beta_{m}$, para magnetização espontânea e susceptibilidade são definidos respectivamente por

- $\beta_{c}$ (ponto final do regime de altas temperaturas)

$$
\beta_{c}=\sup \left\{\beta: \sum_{x}\left\langle\sigma_{0} \sigma_{x}\right\rangle_{\beta, 0}<\infty\right\}
$$

- $\beta_{m}$ (ponto final do regime de baixas temperaturas)

$$
\beta_{m}=\inf \{\beta: M(\beta, 0)>0\}
$$

Lema 7.1.1. No modelo de Ising ferromagnético em $\mathbb{Z}^{d}$ com constantes de acoplamento invariantes por translação e somável temos $\beta_{c} \leq \beta_{m}$.

Demonstração. Pela segunda Desigualdade de Griffits temos

$$
\left\langle\sigma_{0}\right\rangle_{\beta}\left\langle\sigma_{x}\right\rangle_{\beta} \leq\left\langle\sigma_{0} \sigma_{x}\right\rangle_{\beta}
$$

Pela invariância translacional da medida de Gibbs a volume infinito temos para todo $x \in \mathbb{Z}^{d}$ que

$$
\left\langle\sigma_{0}\right\rangle_{\beta}=\left\langle\sigma_{x}\right\rangle_{\beta}=M_{+}(\beta) .
$$

Somando sobre toda a rede a desigualdade (7.7) obtemos da igualdade acima a seguinte desigualdade

$$
\sum_{x}\left(\left\langle\sigma_{x}\right\rangle_{\beta}\right)^{2} \leq \sum_{x}\left\langle\sigma_{0} \sigma_{x}\right\rangle_{\beta} .
$$

Se $\sum_{x}\left\langle\sigma_{0} \sigma_{x}\right\rangle_{\beta}<+\infty$ ( isto implica que $\chi(\beta)<+\infty$ ), a única alternativa para $M_{+}(\beta, 0)$ é ser zero. Como $M$ é não decrescente com respeito a $\beta$, temos que $\beta_{c} \leq \beta_{m}$.

Para mostrar a igualdade destas duas temperaturas inversas críticas ( $\beta_{m}=\beta_{c}$ ) é suficiente mostrar que $M_{+}(\beta)>0$ quando $\beta>\beta_{c}$, já que $M_{+}(\beta)=0$ para $\beta \leq \beta_{m}$, e também $\beta_{c} \leq \beta_{m}$, o que obriga $\beta_{m}=\beta_{c}$. 
Usaremos de agora em adiante as desigualdades diferencias Parciais (DDP's), obtidas nos capítulos anteriores, com a finalidade de mostrar a igualdade entre as temperaturas críticas. Para isso vamos usar a seguinte desigualdade diferencial ordinária (DDO), que é obtida combinando (5.1) e (7.1) para eliminar $\frac{\partial M}{\partial \beta}$,

$$
M \leq \beta h \frac{\partial M}{\partial(\beta h)}+M^{3}+\beta\|J\| M^{3} \frac{\partial M}{\partial(\beta h)} .
$$

Observamos que para obter a DDO acima é necessário também usar em (5.1) a cota $\tanh (\beta h) \leq \beta h$.

Vamos agora mostrar uma aplicação de (7.8).

Exemplo 7.1.1. Se fizermos a suposição (que mais adiante não será necessária) de que para cada $\beta$ fixado que a magnetização exibe um comportamento do tipo lei de potência

$$
M(\beta, h)=c(\beta h)^{s},
$$

onde c é uma constante, obtemos os resultados do Teorema 1 e $\beta_{c}=\beta_{m}$.

De fato, se derivamos $M$ em $\beta h$ e multiplicamos por $\beta h$ temos

$$
\beta h \frac{\partial M}{\partial(\beta h)}=s M
$$

Para $\beta<\beta_{c}, M_{+}(\beta)=0$ e $\chi(\beta, 0)<\infty$ teremos $s=1$ e desigualdade (7.8) não é interessante pois ambos lados se anulam.

Para $\beta=\beta_{c}$, esperamos ter na aproximação (7.9) o expoente $s=\frac{1}{\delta}<1$, com $\delta$ dado por (7.0.4). Assim, sob a hipótese de lei de potências (7.9) em $\beta=\beta_{c}$, temos de (7.8) que

$$
\left(1-\frac{1}{\delta}\right) M-M^{3} \leq \beta_{c}|J| M^{3} \frac{\partial M}{\partial(\beta h)} \leq \beta_{c}\|J\| \frac{M^{4}}{\beta h}
$$

onde a última desigualdade é obtida por (7.2). Afirmamos que a desigualdade (7.10) implica, quando $h \rightarrow 0^{+}$, que $\frac{M^{3}}{h} \geq$ const $>0$. De fato, colocando $M$ em evidência em (7.10) e dividindo esta desigualdade por $\beta_{c}\|J\|$ obtemos

$$
\frac{\beta_{c}}{\beta_{c}|J|}\left(1-\frac{1}{\delta}-M^{2}\right) \leq \frac{M^{3}}{h} .
$$

Como estamos assumindo que $M$ é dada por (7.9) tomando o limite 
quando $h \rightarrow 0^{+}$, temos que $M \rightarrow 0$ e portanto segue da desigualdade acima que

$$
0<\frac{1}{|J|}\left(1-\frac{1}{\delta}\right) \leq \frac{M^{3}}{h}
$$

Usando novamente a expressão (7.9) temos também que

$$
0<\frac{1}{|J|}\left(1-\frac{1}{\delta}\right) \leq\left(c^{3} \beta_{c}^{3}\right)^{\frac{1}{\delta}} h^{\frac{3}{\delta}-1}
$$

considerando que $h$ está tendendo a zero, concluímos que $\delta \geq 3$.

Resultados rigorosos sobre estes limite foram obtidos por [15] no contexto de campo médio do modelo de Ising. Em [15] mostrou-se que $\delta=3$, na aproximação de campo médio e em dimensões altas.

Usando a convexidade de $M(\beta, h)$ em sua segunda variável (a convexidade de $M(\beta, h)$ segue da Desigualdade GKS) podemos verificar, para $\beta>\beta_{m}$, que temos

$$
\left[\frac{\beta h}{M(\beta, h)}\right] \frac{\partial M}{\partial(\beta h)} \leq \frac{\left[M(\beta, h)-M_{+}(\beta)\right]}{M_{+}(\beta)}
$$

De fato, da convexidade de $M$ podemos afirmar para todo $h_{0}<h$ que

$$
\frac{\partial M}{\partial(\beta h)} \leq \frac{\left[M(\beta, h)-M\left(\beta, h_{0}\right)\right]}{\beta h-\beta h_{0}}
$$

e como $M(\beta, h)$ é não decrescente temos imediatamente que

$$
\frac{M\left(\beta, h_{0}\right)}{M(\beta, h)} \frac{\partial M}{\partial(\beta h)} \leq \frac{\partial M}{\partial(\beta h)} \leq \frac{\left[M(\beta, h)-M\left(\beta, h_{0}\right)\right]}{\beta h-\beta h_{0}} .
$$

Tomando o limite quando $h_{0} \rightarrow 0^{+}$segue da desigualdade acima que a afirmação está provada.

Para modelos na classe GS a DDP (5.1) também implica o seguinte limite inferior em $\frac{\partial M^{2}}{\partial \beta}$ para $\beta>\beta_{m}$ :

$$
\left.\frac{\partial M^{2}}{\partial \beta}\right|_{\beta, h=0} \geq(2 \beta)^{-1}\left[1-M_{+}^{2}\left(\beta_{c}\right)\right]
$$

e usando a suposição de lei de potências (7.9) em $\beta=\beta_{c}$,

$$
\left.\frac{\partial M^{2}}{\partial \beta}\right|_{\beta, h=0} \geq(2 \beta)^{-1}\left[1-\frac{1}{\delta}-M_{+}^{2}\left(\beta_{c}\right)\right]
$$


A DDO (7.12) também sugere que $M(\beta, 0)>0$ para cada $\beta>\beta_{c}$, e isso é suficiente para provar a igualdade, temos $\beta_{c}=\beta_{m}$. Uma inspeção rápida em (7.11) também revela que o limite $\hat{\beta} \leq \frac{1}{2}$.

Em [2] foi provado o seguinte teorema que vai ser usado na prova do Teorema 7.0.3.

Teorema 7.1.1. Seja $\left\{M_{L}(\beta, \widehat{h})\right\}$ uma sequência de funções positivas definidas para $\beta, \widehat{h}>0$, crescente e diferenciável em ambos $\beta$ e $\widehat{h}$, e convergindo quando $L \rightarrow \infty$ para função $M(\beta, \widehat{h})$, a que admite uma extensão contínua para $\widehat{h}=0$. Suponha que a função $M_{L}$ satisfaz:

$$
M_{L} \leq \widehat{h} \frac{\partial M_{L}}{\partial \widehat{h}}+\partial M_{L} f\left(M_{L}\right)+a_{1} M_{L}^{\theta} \frac{\partial M_{L}}{\partial \beta}
$$

$e$

$$
\frac{\partial M_{L}}{\partial \beta} \leq a_{2} M_{L} \frac{\partial M_{L}}{\partial \widehat{\beta}}
$$

onde $a_{1}, a_{2}, \theta \in(0, \infty)$ e $f \geq 0$ é uma função continua que com as seguintes propriedades:

i) $f(M) \rightarrow 0$ quando $M$ decresce para 0 ;

ii) $\int_{[0,1]} \frac{f(M)}{M} d M<\infty$.

Se existe $\beta_{0}$ para a qual

$$
\frac{M\left(\beta_{0}, \widehat{h}\right)}{\widehat{h}} \rightarrow \infty, \text { quando } \widehat{h} \downarrow 0,
$$

então para $\widehat{h}$ suficientemente pequeno, temos

$$
M\left(\beta_{0}, \widehat{h}\right) \geq C_{1} \widehat{h}^{\frac{1}{(1+\theta)}}
$$

e além disso, para cada $\beta>\beta_{0}$,

$$
M(\beta, 0) \geq C_{2}\left(\beta-\beta_{0}\right)^{\frac{1}{\theta}}
$$

com duas constantes $C_{1}$ e $C_{2}$ acima sendo positivas.

\section{Demonstração do Teorema 7.0.3.}

Para ver que para o modelo de Ising o Teorema (7.1.1) implica o Teorema (7.0.3), primeiro tomamos $\beta_{0}=\beta_{c}$ de modo que (7.13), com $\theta=2$, é 
garantida por (5.1), isto é,

$$
M \leq \beta h \frac{\partial M}{\partial(\beta h)}+M^{2}\left(\beta \frac{\partial M}{\partial \beta}+M\right)
$$

A hipótese (7.14) é garantida pela desigualdade (7.1), que é a seguinte desigualdade

$$
\frac{\partial M}{\partial(\beta)} \leq \beta|J| M \frac{\partial M}{\partial(\beta h)} .
$$

A condição (7.15) segue de (7.2) que é

$$
\chi=\frac{\partial M}{\partial(\beta h)} \leq \frac{M}{\beta h}
$$

e portanto a divergência de $\frac{M\left(\beta_{c}, h\right)}{\beta_{c} h}$ quando $h \downarrow 0$ está garantida pois, para todo $\widehat{h}>0$ temos da desigualdade de GKS-II que

$$
\chi\left(\beta_{c}, \widehat{h}\right)=+\infty
$$

$\operatorname{Com} \theta=2$, (7.16) fornece a parte (iii) do Teorema 7.0.3 uma vez que (7.0.4) garante que $\delta \geq 3$. Note que (7.17) fornece a parte (ii) do Teorema 7.0.3, isto significa que para todo $\beta>\beta_{0}=\beta_{c}$

$$
M(\beta, 0) \geq C_{2}\left(\beta-\beta_{0}\right)^{\frac{1}{2}}>0 .
$$

Este último fato juntamente com (7.0.4) nos dá que $\hat{\beta} \leq \frac{1}{2}$ e por consequência que $\beta_{c}=\beta_{m}$, que é a unicidade da temperatura crítica encerrando a prova do item (i) do Teorema 7.0.3.

Agora, trabalharemos com os modelos que tem medidas de spin na classe GS. A ideia é transformar a desigualdade (6.14) de forma que ela se adapte ao Teorema (7.1.1) $\operatorname{com} \theta=2$. Vamos procurar substituir alguns dos termos contendo fatores da forma $\beta h$ por uma quantidade proporcional a $M$. Tal quantidade é dada pelo Lema 7.1.2 abaixo. Feito tudo isto obtemos a seguinte desigualdade

$$
M \leq \beta h \frac{\partial M}{\partial \beta h}+\left(\beta+2 \beta_{M F}\right)|J| M^{2}\left(\beta \frac{\partial M}{\partial \beta}+M\right)
$$

Onde $\beta_{M F}$ é o valor de campo médio para $\beta_{c}$, definida abaixo. Com esta desigualdade e com a desigualdades (7.13), $\operatorname{com} \theta=2$, temos que para modelos na classe $G S$ que o Teorema (7.1.1) implica o Teorema (7.0.3). 
A quantidade proporcional a $M$ que nos referimos acima é dada por:

Lema 7.1.2. Dada uma distribuição a priori $\rho_{0}$ na classe GS, seja $S^{2}=$ $\int \varphi^{2} \rho_{0}(d \varphi)$. Então para todo $h$ tal que $\beta h S \leq \varepsilon$, temos que

$$
\beta h \leq\left[\left(\frac{\varepsilon}{\tanh \varepsilon}\right) \frac{\beta_{M F}}{\|J\|}\right] M
$$

$\operatorname{com} \beta_{M F}=\left(\|J\| S^{2}\right)^{-1}$.

Demonstração. Por GKS-II é suficiente mostrar a desigualdade acima para $\tilde{M}=\langle\varphi\rangle_{J \equiv 0}$ pois a magnetização é não decrescente com respeito as constantes de acoplamento. Note que

$$
\frac{\partial \tilde{\mathrm{M}}}{\partial(\beta h)}=\left\langle\varphi^{2}\right\rangle_{J \equiv 0}-\langle\varphi\rangle_{J \equiv 0}^{2} \geq S^{2}-\tilde{\mathbf{M}}^{2}
$$

Integrando esta desigualdade, ficamos com

$$
\frac{\tilde{\mathrm{M}}}{S} \geq \tanh (\beta h S) .
$$

Já que tanh $x / x$ é uma função decrescente de $x$, para cada $\varepsilon \geq \beta h S$ temos

$$
\frac{\tanh (\beta h S)}{\beta h S} \geq \frac{\tanh (\varepsilon)}{\varepsilon} .
$$

Juntando estas duas desigualdades temos

$$
\frac{\tilde{\mathbf{M}}}{S} \geq \beta h S \frac{\tanh (\varepsilon)}{\varepsilon} \Longleftrightarrow \beta h \leq\left[\left(\frac{\varepsilon}{\tanh \varepsilon}\right) \frac{1}{S^{2}}\right] \tilde{\mathbf{M}} \leq\left[\left(\frac{\varepsilon}{\tanh \varepsilon}\right) \frac{1}{S^{2}}\right] M
$$

onde na última desigualdade a direita usamos GKS-II que garante que $\tilde{\mathrm{M}}$ é menor ou igual a $M$ (pois os spins estão acoplados por $J(i-j) \geq 0$ ). Considerando $\beta_{M F} \equiv\left(\|J\| S^{2}\right)^{-1}$ (que é o ponto crítico do modelo de campo médio !) basta multiplicar e dividir a última expressão na desigualdade acima por $\|J\|$ para encerrar a prova do lema. 


\section{Referências Bibliográficas}

[1] M. Aizenman, Geometric analysis of $\varphi_{4}^{2}$ fields and Ising models. Parts I and Part II. Commun. Math. Phys. 86:1-48 (1982).

[2] M. Aizenman and D.J. Barsky, Sharpness of the phase transition in percoloation models. Commun. Math. Phys. 108:489-526 (1987).

[3] M. Aizenman, D.J. Barsky and R. Fernández, The phase transition in a general class of Ising-type modesl is sharp. J. Stat. Phys. 37: Nos. 3/4 (1987).

[4] M. Aizenman, H. Duminil-Copin and V. Sidoravicius, Random currents and continuity of Ising model's spontaneus magnetization. Commun. Math. Phys. (2014)

[5] M. Aizenman and R. Fernández, On the critical behavior of the Magnetization in high-dimensional Ising models. J. Stat. Phys. 16:463-476 (1977).

[6] J. R. Barry, Probabilidade: um curso em nível intermediário. 3 ed. Rio de Janeiro. IMPA. (2013).

[7] L. M. Ciolleti, Comportamento assintótico das funções de correlações para modelos de spins e percolação de alcance misto. Tese de Doutorado, UFMG. (2008).

[8] R. Bissacot e L. M. Cioletti, Introdução à teoria das medidas de Gibbs. Notas de Aula, USP (2012).

[9] G. A. Braga e F. Fontenele, O limite termodinâmico e independência das condições de contorno para o modelo de Ising d-dimensional. Anais da VIII semana da iniciação cientifica da UFMG. Belo Horizonte. (1999). 
[10] G. A. Braga e F. Fontenele, Caracterização da fase desordenada do modelo de Ising d-dimensional via desigualdades de correlação. I semana do conhecimento da UFMG. (2000).

[11] D. Brydges, J. Fröhlich and T. Spencer, The Random Walk Representation of Classical Spin Systems and Correlation Inequalities, Commun. Math. Phys. 83, 123-150, (1982).

[12] E. da Costa, Medidas de Gibbs e o teorema de AizenmanHiguchi.UnB. Dissertação de Mestrado. Universidade de Brasília. (2013).

[13] R. Ellis, Entropy, Large, Deviations, and Stathistical Mechanics. Germany. Springer-Verlag. p 364 (2006).

[14] S. Friedli and Y. Velenik, Equilibrium statistical mechanics of classical lattice system: a concrete introduction, [online] http://www.unige.ch/math/folks/velenik/smbook/ (June 23, 2014).

[15] J. Frohlich and A. D. Sokal, The random walk representation of classical spin systems and correlation inequalities. III. Nonzero magnetic field, Preprint.

[16] H. Georgii, Gibbs measures and phase transitions. 2nd ed. Göttingen. Walter de Gruyter GmbH \& Co. KG. (2011).

[17] J. Glimm and A. Jaffe, Quantum Physics: A Functional Integral Point of View, Second Edition, Springer-Verlag, New York, (1987).

[18] E. H. Lieb, A Refinement of Simon's Correlation Inequality, Commun. Math. Phys., 77,127-135, (1980).

[19] J. Paiva, Correlation Inequalities. Preprint (2013).

[20] B. Simon, Correlation inequalities and the decay of correlations in ferromagnets. Comm. Math. Phys., 77:111-126, (1980).

[21] S. Vasconcelos, Transição de fase difusiva-balística em polímeros aleatórios. Tese de Doutorado. Universidade de Brasilia (2014).

[22] Y. Velenik, Le modèle d'Ising. Université de genève, http://www.unige.ch/math/folks/velenik/cours.html (Version du 25 mai 2009). 